\title{
2020 AOA Research Abstracts and Poster Competition
}

This issue of The Journal of the American Osteopathic Association (JAOA) features abstracts from the posters that were presented at the 2020 Osteopathic Medical Conference and Exposition (OMED20) in the Research Dissemination Track, which took place in virtually on Friday, October 16 and Saturday, October 17, 2020.

This year's abstracts were organized into Basic Science, Clinical, and Health Services categories, indicated within each abstract immediately under the identification number. Abstracts submitted by students for the poster competition (designated with “*”) were judged, and the first- and second-place winners are designated

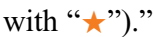

To enhance the readability of this special feature, abstracts have been edited for basic style only. The content has not been modified; information provided reflects information that was submitted by the primary author, including professional degrees and affiliations. Neither the AOA's Bureau of Clinical Education and Research nor the JAOA assume responsibility for the content of these abstracts. (10.7556/jaoa.2020.157)
Poster No. ${ }^{*} \mathrm{C} 13$

Abstract No. 13

Category: Clinical

Research Focus Area: Osteopathic Philosophy

\section{A Retrospective Analysis of Human} Parainfluenza Virus in the Pediatric

\section{Population}

Sarah Elizabeth Straka, OMS IV ${ }^{1}$; Diana Vasilakos, OMS IV ${ }^{1}$; Hanna S. Sahhar, MD²; Kristen Turner, PharmD²;

Rebecca Raffler, DO ${ }^{1}$; Darcy Luck ${ }^{1}$

${ }^{1}$ New York Institute of Technology (NYIT), Old Westbury, NY; ${ }^{2}$ New York Institute of Technology (NYIT), Old Westbury, Spartanburg Regional Healthcare System, Spartanburg, SC

Statement of Significance: Human parainfluenza viruses are among the most common acute respiratory infections in children and contribute significantly to hospitalizations and emergency room visits in the pediatric population. Differences in clinical presentation and sequelae of the four HPIV serotypes have not been thoroughly characterized. This study describes the clinical symptoms, disease severity, and care demands associated with each of the viral serotypes, which may aid in decision making for the most appropriate available treatment modalities for future pediatric patients and improve patient outcomes.

Research Methods: This single-center, retrospective, observational study was conducted by reviewing pediatric patients admitted with parainfluenza virus to the general pediatrics ward or PICU at Spartanburg Regional Hospital from august 2018 to November 2019. Parainfluenza virus presence was confirmed using a polymerase chain reaction based test called FilmArray Respiratory Panel, which identifies common viral and bacterial pathogens that infect the upper respiratory system. Inclusion criteria consisted of all patients less than 18 years of age admitted to the above units with a diagnosis of parainfluenza serotypes 1-4 confirmed via FilmArray Respiratory Panel.

Data Analysis: Patients were identified through data management software and stratified by parainfluenza serotype for data analysis. The following data were collected: HPIV serotype, demographics (age, gender, race, 
zip code), month of diagnosis, past medical history (prematurity, immunodeficiency, prior surgery, congenital heart disease), admission location (general pediatrics vs PICU), length of stay, clinical presentation, disease spectrum, presence of concomitant infection (Respiratory syncytial virus (RSV), influenza, bacterial pneumonia), clinical management, complications during hospital stay, and patient outcome (discharge home, transfer to higher level of care, death). The data were analyzed to identify patterns within the four parainfluenza serotypes, with ANOVA and chisquare tests evaluating statistical significance.

Results: The collected data found nonspecific upper respiratory infection symptoms was the most common clinical presentation across HPIV serotypes, affecting $75 \%$ of patients. While parainfluenza virus is commonly associated with croup, only $35 \%$ of patients across all serotypes were noted to have traditional croup-like symptoms (bark-like cough, inspiratory stridor). The results demonstrated a significant difference between serotypes and manifestation of croup symptoms ( $p=0.015$ ), with HPIV-2 and HPIV-1 demonstrating the highest percentages of patients diagnosed with croup at $66 \%$ and $50 \%$, respectively. The most frequently diagnosed serotype was HPIV-3, and these patients experienced higher rates of bronchiolitis, pneumonia, and upper respiratory infection symptoms compared with other serotypes. While no statistical significance was observed in our small population, serotypes 1 and 4 necessitated greater care demands; with HPIV-1 resulting in the most pediatric intensive care unit (PICU) admissions (50\%) and HPIV -4 patients averaging the longest length of stay and requiring the most supplemental oxygen.

Conclusion: This pilot study suggests that understanding the differences in symptomatology and severity of each serotype can proactively aid in the proper treatment and care of parainfluenza virus in the pediatric population. Further investigative studies with an increased sample size, multiple sites, and longer duration would be beneficial to support the data and further delineate preemptive actions when caring for the pediatric population with HPIV.

\section{Acknowledgment/Funding Source:}

\section{References}

1. Branche, AR, Falsey, AR. Parainfluenza Virus Infection. Semin Respir Crit Care Med. 2016;37(4):538-554. doi:10.1055/ s-0036-1584798

2. Pawełczyk, M, Kowalski, ML. The Role of Human Parainfluenza Virus Infections in the Immunopathology of the Respiratory Tract. Current allergy and asthma reports. 2017;17(3):16. doi:10.1007/ s11882-017-0685-2.

3. DeGroote, NP, Haynes, AK, Taylor, C, et al. Human parainfluenza virus circulation, united states, 2011-2019. Journal of clinical virology. 2020;124. doi:10.1016/j.jcv.2020.104261

4. Abedi, GR, Prill, MM, Langley, GE, et al. Estimates of Parainfluenza Virus-Associated Hospitalizations and Cost Among Children Aged Less Than 5 Years in the United States, 1998-2010. J Pediatric Infect Dis Soc. 2016;5(1):7-13. doi:10.1093/jpids/piu047

5. Frost, HM, Robinson, CC, Dominguez, SR. Epidemiology and clinical presentation of parainfluenza type 4 in children: a 3-year comparative study to parainfluenza types $1-3$. J Infect Dis. 2014;209(5):695-702. doi:10.1093/infdis/j

Poster No. *C14

Abstract No. 14

Category: Clinical

\section{Research Focus Area: Chronic Diseases \& Conditions}

\section{Evaluation of the Emergency Department Management of Children With Diabetic Ketoacidosis}

Alyssa Marian DaVolio, OMS IV ${ }^{1}$; Jacob C. Andrews, OMS IV²; Machenzie Dreher, OMS IV². Brittany Skaggs, OMS IV²; Christine Angeles, OMS $\mathrm{IV}^{2}$; Ning Cheng, $\mathrm{PhD}^{3}$; Hannah Sahhar, $\mathrm{MD}^{2}$ ${ }^{1}$ Edward Via College of Osteopathic Medicine (South Carolina), Spartanburg, SC; ${ }^{2}$ Department of Pediatrics, Edward Via College of Osteopathic Medicine (South Carolina), Spartanburg, SC; ${ }^{3}$ Department of Statistics, Edward Via College of Osteopathic Medicine (South Carolina), Spartanburg, SC 
Statement of Significance: Patients in diabetic ketoacidosis often present initially to the emergency department where they are stabilized with fluids and insulin. After initial workup and stabilization patients are often transferred to the pediatric intensive care unit for further treatment. The purpose of this study is to re-evaluate the management of diabetic ketoacidosis and time flow in the emergency department before and after the implementation of electronic medical records. One of the osteopathic tenets is "The human body is a functional unit. Mind, body, and spirit are interconnected." An analysis of how the body is managed during diabetic ketoacidosis will potentially lead to improvements of the mind, body and spirit of the patient.

Research Methods: A retrospective medical record review of 247 participants between the ages of 0-18 years admitted to the pediatric intensive care unit with a diagnosis of diabetic ketoacidosis and bicarbonate level $<15$ was conducted. Data collected included the participants' gender, age, race, weight, height, initial vitals at emergency department admission, emergency department diagnosis, time from admission to emergency department to decision to admit to the pediatric intensive care unit, time from decision to admit to the pediatric intensive care unit to transfer to the pediatric intensive care unit, fluids $(0.9 \% \mathrm{NaCl})$ given in the emergency department, amount of insulin given in the emergency department, the route of administration (IV drip vs bolus), blood gas results collected, and blood glucose results collected.

Data Analysis: A total of 247 patients were included in this analysis with 154 patients repre- senting care prior to electronic medical records and 93 patients representing care after implementation of electronic medical records. $44.1 \%$ were male, $55.9 \%$ were female, $48.4 \%$ were African American, $48.8 \%$ were Caucasian, $0.4 \%$ were Hispanic, $2 \%$ were Asian, $0.4 \%$ were classified as other. $0.4 \%$ were greater than 17 years, $60.7 \%$ were $13-17$ years, $32.8 \%$ were 6-12 years, and $5.3 \%$ were $1-5$ years. $97.6 \%$ were Type 1 Diabetes Mellitus and 2.4\% were Type 2 Diabetes Mellitus. 28.3\% were experiencing their first instance of diabetic ketoacidosis and $71.7 \%$ were experiencing a recurrence of diabetic ketoacidosis. The average throughput times prior to the use of electronic medical records in the emergency department were as follows: time from admission to the emergency department to decision to admit to the pediatric intensive care unit was 2.02 hours \pm 1.65 . The time from the decision to admit to the pediatric intensive care unit to transfer to the pediatric intensive care unit was 1.15 hours \pm 1.10 . These results were compared with throughput after implementation of electronic medical records in the emergency department, which were as follows: time from admission to the emergency department to decision to admit to the pediatric intensive care unit was 0.66 hours \pm 1.30 . The time from the decision to admit to the pediatric intensive care unit to transfer to the pediatric intensive care unit was 2.10 hours \pm 1.39 .

Results: Comparing the averages between the two groups, we found there was a statistically significant decrease in time from admission to the emergency department to decision to admit to the pediatric intensive care unit of 1.36 hours $(82$ minutes $) \quad(p<0.001)$ after the 
implementation of electronic medical records. Additionally, there was a statistically significant increase in time from the decision to admit to the pediatric intensive care unit to transfer to the pediatric intensive care unit of 0.45 hours (27 minutes) $(\mathrm{p}<0.001)$ after the implementation of electronic medical records.

Conclusion: The overall results of the implementation of the electronic medical records was shown to be interesting. More specifically, the implementation of electronic medical records allowed for quicker time from admission to the emergency department to decision to admit to the pediatric intensive care unit. However, the time from the decision to admit to the pediatric intensive care unit to transfer to the pediatric intensive care unit increased after the implementation of electronic medical records. Overall the data showed there is some promise to having electronic medical records to aid in this process, but further investigation is required to determine the total amount of effectiveness that electronic medical records will serve.

Acknowledgment/Funding Source: This study was approved by Spartanburg Regional Healthcare System Institutional Review Board.

We would like to acknowledge Dr. Sahhar for his assistance, direction, and encouragement as we completed this study. We would also like to acknowledge Dr. Cheng for her assistance in calculating the statistics and our fellow students (Christine Angeles, OMS IV, Mackenzie Dreher, OMS IV, and Brittany Skaggs OMS IV) for their assistance in data collection.
Poster No. *C16

Abstract No. 16

Category: Clinical

Research Focus Area: Osteopathic Philosophy

\section{Retrospective Case Review of Stroke} Readmissions Due to Infection

Shivansh Chawla, OMS II ; Ruth Bailey, BSN, RN²; Amy Beller, BSN, RN, CMSRN, SCRN²; Sarah Keller, MSN, RN-BC, AGCNS-BC, SCRN²; Richard Lukose, DO ${ }^{2}$ ${ }^{1}$ Philadelphia College of Osteopathic Medicine, Philadelphia, PA; ${ }^{2}$ Department of Neurology, Tower Health, Reading, PA

Statement of Significance: During a hospital admission for acute stroke, patient outcomes can be improved with a holistic philosophy. Such an approach may reduce common infections encountered during hospitalizations, reducing patient morbidity and health care costs.

Research Methods: Medical records of 99 Patients at Reading Hospital from January 1, 2019 to June 31, 2020, with final ICD-10 codes of I60, I61, and I63, and readmitted within 30 days were reviewed on EPIC. Data points in the initial report include age, sex, race, ethnicity, medical history, index admission and discharge dates, thirty-day readmission admit and discharge dates, length of stay, length between admissions, discharge disposition and discharge destination. After determining the primary cause of readmission, various factors related to the hospitalization were collected; stroke subtype, alteplase treatment, thrombectomy history, NIHSS-Admission Index, NIHSS-Discharge Index, external catheter usage, straight catheter usage, indwelling catheter usage, urine culture, ventilator usage, history of urinary incontinence, history of chronic disease, dysphagia, diet modifications, and toileting abilities. Formal IRB review was deemed unnecessary by 
the IRB office as the project was classified as a Quality Improvement project.

Data Analysis: Data was organized and analyzed using Microsoft Excel.

Results: Infections were the primary cause of readmission in $34 \%$ of patients at Reading Hospital from January 1, 2019 to June 31, 2020 (34 total infections / 99 total readmission). Of these infections, the majority were due to pneumonia (45\% and 16 patients) or urinary tract infection (47\% and 17 patients). Pneumonia was associated with ventilator usage (25\% of pneumonia patients vs $11.8 \%$ of UTI patients) and a history of chronic lung disease $(37.5 \%$ vs $17.6 \%)$. UTI, on the other hand, was associated with indwelling catheter use $(17.6 \%$ of UTI patients vs $6.25 \%$ of pneumonia patients), under the care of a urologist $(11.8 \%$ vs $0 \%)$, urinary retention (35.3\% vs $18.75 \%)$, and history of UTI (23.5\% vs $6.25 \%)$. Patients readmitted for infection also had a higher NIHSS-Admission Index (8 vs $4.1, \mathrm{p}<0.05$ ).

Conclusion: Patients readmitted for infection had a higher NIHSS-Admission Index, suggesting a more severe initial hospital course. Patients with history of chronic lung disease and who required intubation should be suspected for readmission due to pneumonia. Patients who required indwelling catheter usage, had a history or being under the care of a urologist, or had a history of urinary retention should be suspected for readmission due to UTI. These data may be used to formulate interventions to target at risk populations for readmissions for infections in our stroke population, with the goal of reducing morbidity and health care costs.

Acknowledgment/Funding Source: This project was possible through support from the 2020 Summer Internship Program at Reading Hospital.
Poster No. ${ }^{*} \mathrm{C} 18$

Abstract No. 18

Category: Clinical

Research Focus Area: Osteopathic Philosophy

AOA Grant Number: 1907746

\section{Do Males and Females Exhibit the} Same Physiological Stress Response to Medical School Exams?

Alexander John Hedley Morris, OMS III'; Ravi Chinsky, OMS III2; Danielle Wolin, OMS III²; Arline Allera, $\mathrm{BA}^{3}$; Eleanor Yusupov, DO, $\mathrm{MPH}^{2}$; Joanne Donoghue, $\mathrm{MS}, \mathrm{PhD}^{4}$; Jerry Balentine, $\mathrm{DO}^{1}$; William Blazey, DO ${ }^{2}$; Bhuma Krishnamachari, $\mathrm{PhD}^{2}$ ${ }^{1}$ New York Institute of Technology (NYIT), Old Westbury, NY; ${ }^{2}$ Department of Clinical Specialties, New York Institute of Technology (NYIT), Old Westbury, NY; ${ }^{3}$ Academic Health Care Center, New York Institute of Technology (NYIT), Old Westbury, NY; ${ }^{4}$ Department of Osteopathic Manipulative Medicine, New York Institute of Technology (NYIT), Old Westbury, NY

Statement of Significance: Physician burnout is frequently attributed to stress, and research suggests that stress often begins in medical school. Stress can be chronic or acute, and acute stressors such as examinations are unavoidable due to the need to measure knowledge and proficiency. Acute stress may cause a number of physiological responses including increased heart and respiratory rates. These responses may differ between males and females. The osteopathic philosophy is committed to well-being on all levels and is deeply rooted in the concept of human equality. Understanding stress responses in students and understanding which groups of students are at the highest risk may give us insight into how to create targeted interventions.

Research Methods: In an IRB approved pilot study, 20 first and second year osteopathic 
medical student participants were given a Hexoskin ProShirt, a biometric shirt containing built-in electrocardiogram (ECG) and respiratory sensors allowing measurements of heart and respiratory rate. Of the 20 participants, 10 were born as and identified as male and 10 were born as and identified as female. The medical students wore the shirts from 10 minutes prior to an examination until the end. Measurements were collected at two separate examinations taken 11 days apart, resulting in 40 separate data points.

Data Analysis: The data produced during each examination were uploaded onto Hexoskin's online platform. This was then converted to an excel spreadsheet and data analysis was performed using the JAMOVI software. We examined heart rate and respiratory rate at 1 minute before the examination start time, 1 minute after the examination start time, and 10 minutes after the examination start time.

Two-tailed paired $t$ tests were performed comparing heart rate and respiratory rate at the three time points. The two-tailed paired t tests were repeated as subgroup analyses of first- and second-year students, and male and female students. Next, twotailed independent $t$ tests were performed comparing the heart rate of male and female students at all three time points. The significance threshold was set at $\mathrm{p}=.05$.

Results: From 40 student-examinations we acquired 36 respiratory rates and 38 heart rates. Vital signs were sampled in 1 second intervals. The average of 60 seconds of each vital sign was calculated to generate a respiratory rate or heart rate for each of our time points of interest. Due to technical difficulties the respiratory rate data was either not recorded or obviously mis-measured by the ProShirt in 4 student-examinations, and the heart rates were missing in 2 student-examinations. Two-tailed paired $t$ tests revealed a significant difference $(p<0.01)$ between the heart rate 1 minute prior to examination start and 10 minutes after examination start, with a mean difference of $5.62 \mathrm{bpm}$, and also a significant difference $(\mathrm{p}<0.01)$ between the heart rate 1 minute after examination start and 10 minutes after examination start, with a mean difference of 4.50. There was no significant difference in heart rate 1 minute prior to examination start and 1 minute after examination start. There was no significant difference in respiratory rate between any of the 3 time points. In the subgroup analysis it was found that the significant decrease in heart rate at 1 minute prior to examination start and 10 minutes after examination start, and the significant decrease in heart rate at 1 minute after examination start and 10 minutes after examination start remained in both first and second year students, and male and female students. Two-tailed independent $t$ tests revealed no significant difference found in heart rate and respiratory rate at all three time points between sexes.

Conclusion: These results suggest there is a significant decrease in osteopathic medical students' heart rates from the start of an examination to 10 minutes afterwards. This result appears to be generalizable between both male and female students and does not significantly differ between sex. The observed change in heart rate is likely a physiological response to the stress and anxiety present at the beginning of a high stakes examination. Once the students are further into the examination, their heart rate lowers, likely indicating they are now focused on the examination and no longer actively feeling stressed or worrying about their performance. While these results appear to be common sense based off of the common experience of undergoing high stakes testing, it provides us with confirmation of a physiological stress response we can attempt to reduce with 
targeted interventions. Possible interventions for future study may include education in mindfulness techniques, meditation, and other stress reducing methods.

Acknowledgment/Funding Source: Funding for this pilot study was provided by AOA grant number 1907746 .

\section{References}

1. Worly, B, Verbeck, N, Walker, C, Clinchot, DM. Burnout, perceived stress, and empathic concern: differences in female and male Millennial medical students. Psychol Health Med. 2018;:1-10.

2. Ayala, EE, Winseman, JS, Johnsen, RD, Mason, HRC. U.S. medical students who engage in self-care report less stress and higher quality of life. BMC Med Educ. 2018;18(1):189.

3. Kim, HG, Cheon, EJ, Bai, DS, Lee, YH, Koo, BH. Stress and Heart Rate Variability: A Meta-Analysis and Review of the Literature. Psychiatry Investig. 2018;15(3):235-245.

4. Villar, R, Beltrame, T, Hughson, RL. Validation of the Hexoskin wearable vest during lying, sitting, standing, and walking activities. Appl Physiol Nutr Metab. 2015;40 (10):1019-24.

5. Pion-massicotte, J, Godbout, R, Savard, P, Roy, JF. Development and validation of an algorithm for the study of sleep using a biometric shirt in young healthy adults. J Sleep Res. 2018.

\section{Poster No. C21}

Abstract No. 21

Category: Clinical

Research Focus Area: Chronic

Diseases \& Conditions

Physical Function Over the Treatment Period of Three Randomized

Controlled Phase III Trials of Subcutaneous Tanezumab in Patients With Osteoarthritis

Steven P. Stanos, DO ${ }^{1}$; Wilson J. Chang, MD²; Cory Hultman, $\mathrm{DC}^{3}$; Mojgan Sadrarhami ${ }^{4}$; Takaharu Yamabe, $\mathrm{PhD}^{5}$; Peter W. Park, $\mathrm{PhD}^{4}$ ${ }^{1}$ Swedish Pain Services; ${ }^{2}$ Swedish Pain Services, Physical Medicine and Rehabilitation, Seattle, WA; ${ }^{3}$ Eli Lilly and Company, Indianapolis, IN; ${ }^{4}$ Pfizer Inc., New York, NY; ${ }^{5}$ Pfizer Inc., Groton, CT
Statement of Significance: Osteoarthritis is a common condition that can cause significant pain and physical disability [1]. It is among the top 10 most disabling diseases in developed countries $80 \%$ of patients experience limitations in movement and $25 \%$ are left unable to perform their major daily activities [2]. Estimates of OA prevalence vary but may approach 5\% globally, with around $10 \%$ of men and $18 \%$ of women aged 60 years or over experiencing symptoms [2,3]. Recent treatment guidelines for hip and knee OA reflect the need for additional options, as current standard of care analgesics are ineffective or inappropriate for a number of patients [4,5]. If tanezumab is effective in patients with $\mathrm{OA}$, it may offer an alternative treatment option.

Research Methods: This analysis presents patientlevel physical function time course data from three multinational Phase III clinical studies. All studies enrolled patients with moderate to severe OA of the knee or hip who had inadequate response to, or could not tolerate, standard of care analgesics. The index joint was the most painful at baseline with a qualifying Western Ontario and McMaster Universities Osteoarthritis Index (WOMAC) Pain score $(\geq 5 / 10)$ and Kellgren-Lawrence Grade $(\geq 2)$ as confirmed by the Central Reader. Study 1 [6] (NCT02697773) was a dose-titration study where patients received subcutaneous doses of either: placebo at baseline and week 8; tanezumab 2.5 $\mathrm{mg}$ at baseline and week 8 ; or tanezumab $2.5 \mathrm{mg}$ at baseline and $5 \mathrm{mg}$ at week 8 . In Study 2 [7] (NCT02709486), patients received three subcutaneous doses of placebo, tanezumab $2.5 \mathrm{mg}$, or 5 $\mathrm{mg}$ at baseline, week 8, and week 16. In Study 3 [8,9] (NCT02528188), patients received a stable dose of nonsteroidal anti-inflammatory drug (NSAID) before randomization to double-dummy tanezumab $2.5 \mathrm{mg}$ or $5 \mathrm{mg}$ (at baseline and every 8 weeks during a 56-week treatment period) or twice-daily oral NSAID. Patients completed a 
WOMAC Physical Function subscale questionnaire $(0-10$ with higher score indicating increasingly poorer function) at each clinic visit. All studies were approved by relevant institutional review boards.

Data Analysis: This analysis presents change from baseline in WOMAC Physical Function score throughout the treatment period for all randomized patients. For study 1, this included scores provided at weeks 2, 4, 8, 12 and 16 . Study 2 additionally included week 24 . For study 3 , this included scores at baseline and weeks 2, 4, $8,16,24,32,40,48$, and 56. Data were analyzed using analysis of covariance modelling with multiple imputation for missing values (terms: baseline score, baseline diary average pain, index joint [hip or knee], Kellgren-Lawrence grade, NSAID cohort [diclofenac, celecoxib or naproxen; study 3 only] and treatment group, with study site as a random effect). The least squares (LS) mean (standard error [SE]) change from baseline is shown for each timepoint and significance was calculated vs placebo (studies 1 and 2) or NSAID (study 3).

Results: In total, 4541 patients were evaluated in the three studies (study 1: $\mathrm{n}=696$; study $2: \mathrm{n}=$ 849; study 3: $\mathrm{n}=2996$ ). Throughout the treatment period of study 1 , a significantly better improvement from baseline in WOMAC Physical Function was observed in patients receiving either $2.5(\mathrm{n}=231)$ or $2.5 / 5 \mathrm{mg}(\mathrm{n}=233)$ tanezumab, as compared with those receiving placebo ( $\mathrm{n}=232$; weeks $2,4,8,12$, and 16). Similar findings were observed in study 2 , where there were statistically significant improvements from baseline throughout the 24-week treatment period for both tanezumab treatment groups $(2.5[\mathrm{n}=283]$ and $5 \mathrm{mg}[\mathrm{n}=284])$ vs placebo $(\mathrm{n}=282)$. In study 3 , patients receiving $2.5 \mathrm{mg}$ tanezumab $(\mathrm{n}=1002)$ showed a significantly better improve- ment in physical function from baseline at weeks 2 and 4 as compared with patients receiving an NSAID $(\mathrm{n}=996)$. At weeks 4,8 , and 16 , patients receiving $5 \mathrm{mg}$ tanezumab $(\mathrm{n}=998)$ showed a significantly better improvement from baseline compared with those receiving an NSAID. Differences were not significant at other clinic visits during the 56-week treatment period.

Conclusion: Statistically significant improvements in WOMAC Physical Function were observed across the full treatment period (16 or 24 weeks) in two studies assessing the efficacy of subcutaneous tanezumab vs placebo. In an active comparator study, tanezumab was associated with significant improvements in physical function over NSAID treatment at several time points up to week 16. Improving physical function in $\mathrm{OA}$ is important so that patients are able to maintain or regain their ability to perform important daily activities.

Acknowledgment/Funding Source: These studies were sponsored by Pfizer and Eli Lilly and Company. Editorial support was provided by Jennifer Bodkin of Engage Scientific Solutions and was funded by Pfizer and Eli Lilly and Company.

\section{References}

1. Neogi, T. Osteoarthr Cartil. 2013;21(9):1145-33.

2. World Health Organization. Chronic diseases and health promotion; Chronic rheumatic conditions. https://www.who.int/ chp/topics/rheumatic/en/. Accessed 27 May 2020.

3. Cross, M. et al. Ann Rheum Dis. 2014;73(7):1323-30.

4. Kolasinski, SL. et al. Arthritis Rheumatol. 2020;72(2):220-33

5. Bannuru, RR. et al. Osteoarthr Cartil. 2019;27(11):1578-89.

6. Schnitzer, T. et al. JAMA. 2019;322(1):37-48

7. Berenbaum, F. et al. Ann Rheum Dis. 2020;79(6):800-10.

8. Hochberg, MC. et al. Arthritis Rheumatol. 2019;71(s10). Abstract 1302.

9. Hochberg, MC. et al. Arthritis Rheumatol. 2019;71(s10). Abstract 2756 . 
$\star$ Poster No. ${ }^{*} \mathrm{C} 26$

Abstract No. 26

Category: Clinical

Research Focus Area: Chronic

Diseases \& Conditions

Multisystem Inflammatory Syndrome

in Infant With Negative SARS-CoV-2

RT-PCR and Antibodies

Karly Derwitz, OMS IV; Hanna S. Sahhar, MD

Edward Via College of Osteopathic Medicine (South

Carolina), Spartanburg, SC

Statement of Significance: Since the declaration of the SARS-CoV-2 pandemic in March 2020 by the World Health Organization, there has been an emergence of a new syndrome termed Multisystem Inflammatory Syndrome in Children (MIS-C) associated with coronavirus disease (COVID-19). We document this case to aid in the understanding of this syndrome and provide guidance for similar cases encountered by osteopathic physicians in the future. To the best of our knowledge and through extensive research, we are reporting the youngest pediatric patient with the diagnosis of MIS-C. According to the Centers for Disease Control and Prevention (CDC), this is also the first reported case of MIS-C in South Carolina. ${ }^{1}$

Research Methods: A 3-month-old African American male with no significant medical history presented with a two-day history of fevers, abdominal pain, dry cough, and diarrhea. Illness started with fever and erythematous rash on the face and chest with papules on the torso and axilla. The following day, he presented to the emergency department with worsening fever, bilateral conjunctivitis and purulent eye discharge. He was diagnosed with a viral illness during both presentations and discharged home on supportive care. The patient returned on day 5 of his illness with decreased oral intake, decreased urine output and about $10 \%$ body weight loss over 2 days. On physical examination, the patient was illappearing, nontoxic, tachycardic with dry lips and tender abdomen diffusely. He was not in respiratory distress and lungs were clear to auscultation. Data collection was obtained through the following: complete blood count, complete metabolic panel, C-reactive protein (CRP), arterial blood gas, cerebrospinal fluid (CSF) studies, Filmarray respiratory panel test, SARS-CoV-2 RT-PCR, antigen, IgG and IgM, chest x-ray, blood culture, urinalysis, urine culture, stool PCR, echocardiogram, abdominal ultrasound, D-dimer, ferritin, troponin, triglycerides, liver function tests, erythrocyte sedimentation rate (ESR), and procalcitonin. The choice of studies was determined by the criteria for MIS-C according to the CDC guidelines[2] and included additional studies required to rule out other source of infection.

Data Analysis: MIS-C is defined by the presence of fever for more than 24 hours, systemic inflammation, and multi-organ dysfunction in the context of COVID-19 positivity or known exposure within four weeks of onset of symptoms. ${ }^{2}$ Our patient was admitted to the hospital on day 5 of his fever. Laboratory values supporting signs of inflammation included reduced lymphocytes at $8 \%$, hypoalbuminemia of $2.5 \mathrm{~g} / \mathrm{dL}$, elevated CRP of $24.90 \mathrm{mg} / \mathrm{dL}$, ESR of 82 millimeters/hour, and D-dimer of $3.54 \mathrm{ug} / \mathrm{mL}$. Multisystem organ involvement included the gastrointestinal, dermatological and neurological systems due to diarrhea, rash and aseptic meningitis confirmed by CSF results. A full sepsis workup provided there 
was no explanation for other source of infection. Echocardiogram was performed to assess cardiac function and coronary artery involvement and revealed normal results. Evaluation for Kawasaki disease showed purulent conjunctivitis rather than serous, lack of cervical adenopathy, lack of strawberry tongue or mucosal changes and lack of extremity swelling. Gallbladder ultrasound was ordered to rule out hydrops of the gallbladder, which revealed gallbladder wall thickening and pericholecystic/perisplenic edema. SARS-CoV-2 RT-PCR, SARS-CoV-2 antigen, SARS-CoV-2 IgG and $\operatorname{IgM}$ were all negative, but we believe there may have been unknown exposure within 4 weeks of onset of symptoms. There were no recent illnesses in any family member, although the father reported a positive COVID-19 case at place of employment.

Results: As the patient met all of the criteria for diagnosis of MIS-C, the decision was made to start IVIG infusion. The patient showed significant clinical improvement, and laboratory values began to normalize. High dose aspirin $(80 \mathrm{mg} / \mathrm{kg} /$ day $)$ was given until he remained afebrile for 48 hours. There was an increase in white blood cell count, decrease in hemoglobin, and decrease in albumin following treatment demonstrating the normal effect of IVIG. CRP decreased markedly. The patient was discharged home on aspirin $5 \mathrm{mg} / \mathrm{kg} /$ day for 2 weeks until follow up visit with repeat echocardiogram and ESR.

Conclusion: The number of cases of MIS-C are expected to increase as the SARS-CoV-2 pandemic progresses. Our understanding of the underlying pathophysiology and potential mani- festations will improve with additional reports and research. The clinical presentation of MIS-C is variable, with the majority of cases reporting significant gastrointestinal symptoms, cardiac disease, mild or absent respiratory symptoms, rash, conjunctivitis, and oral mucous membrane changes. Limitations of our study include absence of COVID-19 diagnosis due to two negative RT-PCR tests, negative antigen test and negative IgG and IgM test. There also was no presence of COVID-19 illness in the family reducing the likelihood of exposure in this patient. The timing and sensitivity of available SARS-CoV-2 tests may affect the accuracy of the results and unknown exposure to COVID-19 should not exclude MIS-C from the differential diagnosis. Treatment with IVIG in the absence of complete or incomplete Kawasaki disease, shock, or cardiac involvement was successful. Given that the understanding of MIS-C is still evolving, it is important to closely follow potential MIS-C patients as the physical examination findings do not appear simultaneously but rather evolve over several days. Increased index of suspicion and early decision to initiate intensive care are critical in successfully treating MIS-C. If MIS-C goes undiagnosed the deterioration can be quite rapid and severe resulting in significantly increased mortality rates.

\section{References}

1. Infographic: Early Cases of MIS-C: Multi-System Inflammatory Syndrome in U.S. Children. Centers for Disease Control and Prevention. https://www.cdc.gov/coronavirus/2019-ncov/ covid-data/infographic-mis-c.html. Published July 16, 2020. Accessed July 20, 2020.

2. CDC. Multisystem Inflammatory Syndrome in Children (MIS-C) Associated with Coronavirus Disease 2019 (COVID—1919). Emergency Response and Preparedness website. https:// emergency.cdc.gov/han/2020/han00432.asp, 2020. 
Poster No. ${ }^{*} \mathrm{C} 27$

Abstract No. 27

Category: Clinical

Research Focus Area: Impact of Osteopathic Manipulative Medicine (OMM) \& Osteopathic Manipulative Treatment (OMT)

AOA Grant Number: 2891807729

\section{The Effects of Osteopathic} Manipulative Treatment on HbA1c in Type 2 Diabetes and Pre-diabetes: A Randomized-Control Clinical Trial Sudipta Sureshbabu, OMS III ${ }^{1}$; Karen Sheflin, DO²; To Shan Li, DO ${ }^{3}$; Mathew Heller, DO ${ }^{2}$;

Eleanor Yusupov, DO ${ }^{4}$; Bhuma Krischnamachari, $\mathrm{PhD}^{5}$; Arline Allera, BA ${ }^{6}$; Sonia Rivera-Martinez, $\mathrm{DO}^{7}$ ${ }^{1}$ New York Institute of Technology (NYIT), Old Westbury, NY; ${ }^{2}$ Department of Family Medicine, New York Institute of Technology (NYIT), Old Westbury, NY; ${ }^{3}$ Department of OMM, New York Institute of Technology (NYIT), Old Westbury, NY; ${ }^{4}$ Department of Clinical Specialties, New York Institute of Technology (NYIT), Old Westbury, NY;

${ }^{5}$ Department of Research, New York Institute of Technology (NYIT), Old Westbury, NY; ${ }^{6}$ Academic Health Care Center, New York Institute of Technology (NYIT), Old Westbury, NY; ${ }^{7}$ Department of Family Medicine, New York Institute of Technology (NYIT), Old Westbury, NY

Statement of Significance: In the United States, T2DM is a major cause of morbidity and mortality. The prevalence of this disease continues to rise despite advances in medical management. Diabetes is commonly managed using lifestyle modifications and pharmacological treatment, most commonly, metformin and insulin. Despite these strategies, lifetime control of blood glucose is both difficult and expensive. OMT techniques have been demonstrated by Dr. Bandeen, in
Osteopathic Prof. published in 1949, to decrease blood glucose levels in diabetic patients. With increasing insulin prices and prevalence of diabetes, modalities such as OMT can enhance $\mathrm{HbA} 1 \mathrm{c}$ control and mitigate financial expenses by reducing insulin requirements for patients.

Research Methods: This randomized-control clinical trial was reviewed and approved by NYIT IRB (IRB number: BHS-1383). In this ongoing clinical trial, participants with or without prediabetes or T2DM diagnosed at least 5 years prior were enrolled in the study. The participants were blinded and randomized to two groups, control vs treatment. The control group received sham OMT and the treatment group received OMT demonstrated by Dr. Bandeen to treat diabetics. The techniques used in the treatment group include suboccipital release, thoracic outlet release, rib raising (left ribs 2-9, right ribs 5-12), ribless rib raising (right L1-L2), chapman's points for pancreas and liver, fascial stretch of the pancreas in longitudinal axis, test and treatment of pancreatic motility, pancreatic fascial technique, pancreatic stimulation through angle of ribs 3-5, celiac ganglion inhibition, and liver pump. The study was conducted over 6 treatment sessions which were scheduled every 2 weeks. Blood glucose levels were collected twice per treatment session (pre- and post-treatment) for a total of 12 times. HbA1c was collected during the 1 st, $2 \mathrm{nd}$, 4th, and 6th treatment sessions. In addition, vitals were also performed before treatment on all participants. Furthermore, all participants continued with their recommended diabetic medication regimens and medical management as prescribed by their primary provider. There were also no changes in diet or physical activity. 
Data Analysis: All data measurements were inputted into NYITCOM RedCap. At the time of analysis all data was extracted from RedCap into an excel spreadsheet and analysis was conducted using SAS software. All patients were analyzed using intention-to-treat methods. We stratified the participants in each group by sex and diabetes diagnosis (healthy, prediabetes, or diabetes). A repeated measures ANOVA was conducted comparing $\mathrm{HbAlc}$ values between control and treatment within each stratification category. Significance was determined by a threshold of $(\mathrm{p}<0.05)$

Results: A total of 48 participants were included in this analysis (Healthy: $n=15$, Prediabetic: $\mathrm{n}=10$, Diabetic: $\mathrm{n}=23$ ). The study enrolled 29 females and 19 males. The mean pre-treatment and post-treatment $\mathrm{HbAlc}$ values for healthy females were $4.9 \%$ and $5.1 \%$, respectively. The mean pre-treatment and post-treatment $\mathrm{HbA} 1 \mathrm{c}$ values in healthy males were $5.1 \%$ and $4.9 \%$, respectively. The mean pre-treatment and posttreatment $\mathrm{HbA} 1 \mathrm{c}$ values in prediabetic females were $5.3 \%$ and $5.1 \%$, respectively. The mean pretreatment and post-treatment $\mathrm{HbA} 1 \mathrm{c}$ values in prediabetic males were $5.3 \%$ and $5.1 \%$, respectively. The mean pre-treatment and post-treatment HbA1c values in diabetic females were $7.1 \%$ and $6.0 \%$, respectively. The mean pre-treatment and post-treatment $\mathrm{HbA} 1 \mathrm{c}$ values in diabetic males were $8.4 \%$ and $7.2 \%$, respectively. There was a significant difference in $\mathrm{HbAlc}$ between the two treatment groups across visits in healthy females $(\mathrm{p}=0.0268)$ and in prediabetic and diabetic males ( $p=0.0357$ ). In addition, there was a significant difference in $\mathrm{HbA} 1 \mathrm{c}$ across visits in prediabetic and diabetic females $(\mathrm{p}=0.0052)$ but no significance between the two treatment groups.

Conclusion: These results suggest that there was a decrease in the mean $\mathrm{HbA1c}$ post-treatment in the both males and females diagnosed with prediabetes or T2DM. Furthermore, there was significant difference between the sham treatment and OMT in healthy females and prediabetic and diabetic males. The data suggests that OMT can be a useful treatment modality to help patients reach target $\mathrm{HbA} 1 \mathrm{c}$ in the prediabetic and diabetic populations. The study is limited by the small population size and loss of follow-up in patients. Further evaluation on the effects of OMT in aiding in the treatment of Type II diabetes mellitus is warranted as the management of this disease becomes more difficult due to increasing insulin prices and unwanted side-effect profiles.

Acknowledgment/Funding Source: Funding for this clinical trial was provided by AOA grant number 2891807729.

\section{References}

1. Bandeen, SG. Diabetes: Report covering twenty-five years research on stimulation of pancreas, blood chemical changes Osteopath Prof. 1949;17(1):11-15, 38-47

2. Johnson, AW, Shubrook, JH. Role of Osteopathic Structural Diagnosis and Osteopathic Manipulative Treatment for Diabetes Mellitus and Its Complications. JAOA 2013;113(11):829-836

3. Licciardone, JC, Fulda, KG, et al. A case-control study of osteopathic palpatory findings in type 2 diabetes mellitus. Osteopath Med and Primary Care 2007;1(6):1-12

4. Nelson, KE, Mnabhi, AKS, Glonel, T. The accuracy of diagnostic palpation: the comparison of soft tissue findings with random blood sugar in diabetic patients. Osteopathic Family Physician 2010;2:165-169

5. Shubrook, JH. An Osteopathic Approach to Type 2 Diabetes Mellitus. JAOA 2011;111(9):531-537 
Poster No. ${ }^{*} \mathrm{C} 31$

Abstract No. 31

Category: Health Services

Research Focus Area: Chronic

Diseases \& Conditions

Perspectives on Health care Decisions and Total Joint Arthroplasty from the Southside of San Antonio, a Majority Minority Region

Katelyn Jane Franck, OMS III ${ }^{1}$; Margaret E. Jonas, OMS III ${ }^{1}$; Jaydee J. Foster, MA $^{2}$; Roberto J. Fajardo, $\mathrm{PhD}^{3}$; Matthew Morrey, $\mathrm{MD}^{4}$; Scott E. Smith, $\mathrm{PhD}^{5}$

${ }^{1}$ University of the Incarnate Word School of Osteopathic Medicine, San Antonio, TX; ${ }^{2}$ Department of Research and Innovation, University of the Incarnate Word School of Osteopathic Medicine, San Antonio, TX; ${ }^{3}$ Department of Clinical and Applied Science Education, University of the Incarnate Word School of Osteopathic Medicine, San Antonio, TX; ${ }^{4}$ Ortho San Antonio, San Antonio, TX; ${ }^{5}$ Department of Mathematics and Statistics, University of the Incarnate Word School of Osteopathic Medicine, San Antonio, TX

Statement of Significance: TKA is a common and successful OA treatment that significantly reduces pain level and improves quality of life. Women have a higher prevalence of knee arthritis but are less likely to undergo an arthroplasty than men.

This study encompasses behavioral model of osteopathic medicine, investigating mental, emotional, and spiritual impacts on patient care. In order to rectify TKA underuse it is critical to understand patient perspectives on their own health care and decisions regarding OA treatment. Impediments to patient care could be financial, logistical, emotional, spiritual, and other stressors. A better understanding of these factors is necessary to more effectively counsel patients on their health care and OA treatment options.

Research Methods: This IRB approved study consisted of surveys with a combination of spe- cific and Likert scale questions. Participants were recruited at community centers and events around Districts 3 and 4 on the southside of San Antonio, Texas. The participants were 50-89 years old, Bexar County residents, and fluent in English. Responses were kept anonymous.

Data Analysis: Of the 113 participants, $62.5 \%$ identified as Hispanic, $98 \%$ of participants have health insurance, $78.5 \%$ indicated their insurance does a good job of covering their health care needs, and $73.2 \%$ were comfortable with their medical knowledge. Among those who reported daily hip/knee pain, $47.1 \%$ indicated financial concerns would prevent them from pursuing surgery. Half believed joint replacement surgery is common and successful for hip and knee arthritis, but 40.8 and $43.1 \%$, respectively reported they would avoid a total joint arthroplasty (TJA) due to fear or cost even though surgery could alleviate joint pain.

Results: Sex-based comparisons indicated females were more likely to choose a doctor based on geographic proximity $(\mathrm{p}=0.021)$, were less likely to ask for help $(\mathrm{p}=0.006)$ and prefer their health does not affect others $(\mathrm{p}=0.004)$. Men were more comfortable with help making health care decisions $(p=0.008)$. Although females reported a higher rate of clinical diagnosis of knee osteoarthritis $(\mathrm{p}=0.037)$, there was no significant difference in reporting of daily pain. Among participants with daily knee pain, a strong trend indicated that females were less likely to be influenced by another's surgical experience or outcome $(\mathrm{p}=0.107)$. Compared with NHW, Hispanics were more likely to report an above average high pain tolerance $(\mathrm{p}=0.035)$, fear of TJA ( $p=0.007)$, and an avoidance of TJA $(p=0.047)$.

Conclusion: These results provide a glimpse into the complexity of the patient health care 
experience in San Antonio. Although most participants have health insurance, finances still influence a patient's medical decision-making process. While patients perceive joint replacement surgery favorably, fear, costs, and doubts about the benefit of joint replacement surgery appear to drive the decisions to not pursue surgery.

Acknowledgment/Funding Source: We would like to thank San Antonio Districts 3 and 4 council women Rebecca Viagran and Adriana Rocha Garcia's offices for their support as well as the Harvey Najim YMCA, Cisneros and Southside Lions Senior centers, and District 3 farmers market for allowing us to recruit survey participants at their locations.

Poster No. ${ }^{*} \mathrm{C} 32$

Abstract No. 32

Category: Clinical

Research Focus Area: Osteopathic Philosophy

\section{Levels of Resilience \& Coping of Osteopathic Medical Students during COVID-19: Implications for Mental Health Innovations for Physicians in Training}

Michelle Elizabeth Lanspa, OMS II, MBA; Robin Jacobs, PhD, MSW, MS, MPH Nova Southeastern University Dr. Kiran C. Patel College of Osteopathic Medicine, Fort Lauderdale, FL

Statement of Significance: In addition to fears about one's own health, which consequentially precipitate changes in health and coping behaviors, medical students have added concerns regarding the interruption of their studies. While both allopathic and osteopathic students must complete a minimum number of hours of in-person training before clinical rotations, osteopathic medical education also requires 300 hours of training in Osteopathic Manipulation Therapy (OMT). Given the restrictions imposed by COVID-19, concerns of keeping one's skills practiced for board examinations, professional competence in OMT, and uncertainty regarding education timelines are significant sources of stress and raise concerns regarding the emotional wellbeing of osteopathic medical students.

Research Methods: Data were collected from 202 medical students via an online questionnaire developed by the researchers titled Emotional Wellbeing in Medical Students Questionnaire (EWB-Q). Anecdotal evidence and published reports guided the development of the major study variables and the creation of the EWB-Q, which included validated measures to assess the levels of coping strategies, personal resilience (the ability to "bounce back" after a setback), and certain health behaviors (i.e., healthy eating and exercise patterns) among osteopathic medical students and how these variables may contribute to their emotional wellbeing. Data on participants' sociodemographic characteristics were also collected. The study was approved by the researchers' university Institutional Review Board. The survey took approximately 10 minutes to complete.

Data Analysis: Resilience. The 6-item Brief Resilience Scale (BRS) assesses the ability to bounce back or recover from stress and is negatively related to anxiety, depression, negative affect, and physical symptoms when other resilience measures and optimism, social support, and Type D personality are controlled. Responses are scored on a five-category ordinal scale, with higher scores indicating higher levels of resilience.

Coping. The Brief COPE is a 28 -item scale with scores ranging from 1 to 4 , with higher scores indicating greater use of coping strategies. Responses ranged from 1 = I haven't been doing 
this at all, and $4=$ I've been doing this a lot. Categories under which items are reflected include, but are not limited to, self-distraction, active coping, denial, use of emotional support, positive reframing, acceptance, and religion.

Emotional wellbeing. Higher Mental Health Continuum (MHC-SF) is a 14-item scale with scores ranging for 1 to 6 . People can be classified as flourishing (higher scores) or languishing (lower scores) in regard to emotional wellbeing. Responses are scored on a six category ordinal scale ranging from $1=$ never, to $6=$ every day.

Health behaviors. These items were scored using a 4-point ordinal scale where $1=$ haven't been doing this at all, 2 = I've been doing this a little bit, 3 = I've been doing this a medium amount, and $4=$ I've been doing this a lot.

Results: The mean age of the participants was 26.7 years (range $21-57$ years; $\mathrm{SD}=4.05$ ); $59.1 \%$ $(n=110)$ were women. About half $(n=110$; $54.5 \%)$ of participants were in preclinical training (years 1 and 2) and the remainder ( $\mathrm{n}=92 ; 45.5 \%)$ were currently in clinical rotations in outpatient settings/hospitals (years 3 and 4). Multiple linear regression analysis indicated that the level of coping, personal resilience, and certain health behaviors (i.e., eating and sleeping well) explain a significant amount of the variance in emotional wellbeing scores in U.S. osteopathic medical students during the first months of the COVID-19 pandemic in the United States. A significant regression equation was found, $\mathrm{F}(4,171)=$ $17.481, \mathrm{p}<.000, \mathrm{R} 2=.290, \mathrm{R} 2$ adjusted $=.274)$. Higher levels of resilience, greater use of coping strategies, getting enough sleep, and eating well made significant contributions to emotional wellbeing.

Conclusion: This initial study will help guide efforts to develop mental health interventions for osteopathic medical students. The findings can also help to identify and address any novel or changing mental health needs of U.S. osteopathic medical students due to COVID-19, and to evaluate any suggested changes their educational environments and career progression planning. As the importance of supporting mental health among health care providers continues to be an area of professional concern, it is especially relevant during crises and pandemic to consider the mental health of medical students. Cultivating emotional wellbeing should continue to be a goal for educators and administrators of medical schools now more than ever as they design curriculum and campus-based initiatives to help students bolster their skills in personal resilience and encourage healthy coping behaviors. While this is particularly evident during times of crises and education and professional uncertainty, better mental health training as a proactive stance to promote personal resilience is critical for medical students who will soon be providing front-line patient care and influencing health care policy. Osteopathic medical schools and educators should be providing a model for comprehensive care through compassionate and holistic evaluations of students' physical and mental therapy health needs in order to produce providers capable of dealing with an increasingly uncertain and unstable health care environment.

\section{References}

1. Brazeau, CM, Shanafelt, T, Durning, SJ, Massie, FS, Eacker, A, Moutier, $\mathrm{C}$, et al. Distress among matriculating medical students relative to the general population. Academic Medicine 2014;89:1520-25

2. Goebert, D, Thompson, D, Takeshita, J, Beach, C, Bryson, P, Ephgrave, $\mathrm{K}$, et al. Depressive symptoms in medical students and residents: a multischool study. Academic Medicine 2009;84:236-41.

3. Dyrbye, LN, Thomas, MR, Shanafelt, TD. Systematic review of depression, anxiety, and other indicators of psychological distress among U.S. and Canadian medical students. Academic Medicine 2006;81:354-73. 
4. Cao, W., Fang, Z., Hou, G., Han, M., Xu, X., Dong, J., et al. 2020. The psychological impact of the COVID-19 epidemic on college students in China. Psychiatry Res. 285, 112934.

5. Houpy, J, Lee, W, Woodruff, J, Pincavage, A. (2017) Medical student resilience and stressful clinical events during clinical training. Medical Education Online, 22:1, 1320187, DOI: 10.1080/10872981.2017.1320187

Poster No. ${ }^{*} \mathrm{C} 33$

Abstract No. 33

Category: Basic Science

Research Focus Area: Chronic Diseases \& Conditions

\section{Does Chronic Stress Affect Fertility and Survival Rate Among Laboratory Mice and Their Offspring? \\ Lindsey Leggett, OMS III ${ }^{1}$; Jessica F. Brinkworth, $\mathrm{PhD}^{2}$; Kimberly A. Congdon, $\mathrm{PhD}^{1}$ \\ ${ }^{1}$ Touro University Nevada, Henderson, NV; ${ }^{2}$ Department of Anthropology, University of Illinois Urbana-Champaign, Champaign, IL}

Statement of Significance:Fertility rates in America have been declining for several years. Numerous factors have been implicated including the increasing age of women during their first pregnancy pointing to a decline in ovarian reserve as a possible factor. It is often suggested that stress plays a role as well, but the impact of distress in human samples has been difficult to investigate. In this study, we seek to apply the osteopathic holistic approach to the scientific question of evaluating potential factors for this decrease in fertility rates. Should stress be deemed a factor, this would be incredibly significant information to the osteopathic physician and make this a priority when treating the patient to ensure the appropriate interdisciplinary care.

Research Methods: Mus musculus were kept within control and experimental stressed groups. There were 2 females and 1 male within each cage. Animals were socially stressed by changing breeding harems and cages every few days. Animals bred and once the offspring finished nursing, were kept and monitored within these same conditions. The interval length between each litter as well as the survival rates of the offspring were recorded. Preliminary data from 3 parent generation mice of both study groups, 2 first generation mice from both study groups, 1 second generation mice of the control group, and 3 second generation mice of the experimental group was examined for the purposes of this study. IRB approval for this study was obtained.

Data Analysis: Data was analyzed for statistical significance using the Student's 2 sample T test and ANOVA.

Results: The parent generation of stressed mice had an average offspring survival rate of $75.3 \%$ vs the control parent generation rate of $74.9 \%(p=0.98)$. The experimental stress parent group had a mean interval litter length of 37.8 days vs the control parent generation at 28.6 days $(\mathrm{p}=0.12)$. The first generation of stressed offspring had a mean survival rate of $37.5 \%$ vs the same control group with a rate of $85 \%$ ( $p=0.014)$. This first generation of stressed offspring also had a mean inter-pregnancy interval length of 33.7 days vs 25.9 days in the same control group ( $\mathrm{p}=0.20$ ). Lastly, the second generation of stressed offspring mice had a mean offspring survival rate of $97 \%$ vs the same control group at $56.3 \%$ $(\mathrm{p}=0.21)$. The inter-pregnancy interval length between these two groups was 39 days for the stressed group and 37 days for the control $(p=0.89)$.

Regarding the comparison of mean survival rates among generations of stressed mice, there was a p-value of 0.001 vs the control groups had a $\mathrm{p}$-value of 0.41 . Comparing the inter-pregnancy interval length among the generations of stressed mice yielded a p-value of 0.77 vs the control groups with a p-value of 0.38 .

Conclusion: The first generation of stressed offspring appear to be an outlier in that the survival 
rate of their offspring provided the only statistically significant difference among groups. It is possible that these first offspring were the most negatively affected by either their own stress or by their parent's stress and thus were unable to overcome the challenges associated with a successful pregnancy. This would indicate that the second generation of offspring is able to overcome this generational chronic social stress to produce more appropriately viable offspring. Although the stressed groups had an overall increase in interval length between pregnancies compared with their control counterparts, this difference was not statistically significant. Our data is limited by the small sample sizes and because we did find trends in data, but little statistical significance, we question whether a larger power size would have yielded statistically significant results. We believe that this data warrants further investigation with a larger sample size.

\section{$\star$ Poster No. ${ }^{*}$ C35}

Abstract No. 35

Category: Clinical

\section{Research Focus Area: Chronic Diseases \& Conditions}

Traumatic Falls, Comorbid Diseases, Hospital Stays and Discharge: Does a Disease Make the Difference?

Samuel Gregerson, OMS IV ${ }^{1}$; Mandip Atwal, DO ${ }^{2}$ ${ }^{1}$ Michigan State University College of Osteopathic Medicine, East Lansing, Ml; ${ }^{2}$ Department of Surgery, McLaren Macomb Hospital, Mount Clemens, MI

Statement of Significance: Falls lead to diminished function and loss of mobility, accounting for nearly half of the traumas reported in the United States. As falls recently ranked among leading conditions that altered life expectancy and the second leading cause of death due to unin- tentional injury, the importance of fall prevention cannot be overstated. Disease prevention and the maximization of quality of life is an important osteopathic practice. This study identified patient populations that more often required complicated discharge assignments compared with healthy patients after a traumatic fall. These findings can help lead to proactive safeguards against falls in these populations and may ultimately help reduce health care costs.

Research Methods: After IRB approval, a retrospective review of all posttraumatic fall patients presenting to our institution's emergency department dating from January 2016 to December 2017 was performed. The hospital's electronic trauma data base was utilized to obtain the presence of comorbid disease, hospital length of stay and discharge assignment for each patient. At our institution, patients received discharge assignments based on a multidisciplinary medical team approach; this included physical therapy evaluations, coordination with a case manager and communication amongst physicians. Discharge assignment options included: home with selfcare/no additional medical services; home with services; inpatient rehab; skilled nursing facility; acute care facility; long-term care and hospice. In this study, patients were assigned either a "routine discharge" or a "more complicated discharge". A routine discharge was a discharge assignment of home self-care/no additional medical services. Any other discharge assignment, as listed previously, was considered a more complicated discharge because it required some level of additional medical resource. We attempted to standardize traumatic falls for analysis by only including falls under 1 meter in height from the ground. The study age range was limited to 20-70 years old to provide some control over age-related confounding variables of the 
very young and very old. Diabetes, hypertension and smoking were the comorbid conditions included in the study.

Data Analysis: Our study included 6 different patient groups: healthy (no comorbid conditions); diabetes; hypertension; smoker; hypertension and smoker; hypertension and diabetes. In all, our study included 921 patient falls. Statistical analysis consisted of student's two sample $t$ tests to analyze hospital length of stay data. Chi-square tests were calculated to compare the discharge data in each patient cohort compared with healthy patients. P-values less than 0.05 were deemed significant; p-values less than 0.01 were deemed highly significant.

Results: We found no difference in average hospital length of stay between the 6 groups. Next we asked if the patient groups varied in hospital discharge patterns. Smokers and smokers with hypertension displayed a similar discharge pattern compared with healthy patients. Patients with diabetes [X2: 12.55 ( $\mathrm{p}$ value $<0.001)$ ], hypertension $[\mathrm{X} 2: 4.39(\mathrm{p}$ value $=0.04)]$, or a combination of both conditions [X2: 7.16 ( $\mathrm{p}$ value $=0.007)]$ received a higher proportion of complicated discharges compared with healthy patients.

Conclusion: Our results indicated that comorbidities including hypertension and diabetes are associated with a higher proportion of complicated hospital discharge assignments in posttraumatic fall patients compared with healthy posttraumatic fall patients. This data suggests the importance of fall prevention and education in these particular patient populations.

Acknowledgment/Funding Source: The authors report no conflicts of interest to report.
Poster No. *C38

Abstract No. 38

Category: Health Services

Research Focus Area: Osteopathic

Philosophy

\section{Health Literacy in Underrepresented} and Underserved Adolescents

Ashley Pinckney, OMS IV, MBS ${ }^{1}$; Salma Mami Maryanne Lubas, OMS IV ${ }^{1}$; Sandra Ross, LSW ${ }^{3}$; Liana Gefter, MD, MPH ${ }^{4}$

${ }^{1}$ Philadelphia College of Osteopathic Medicine, Philadelphia, PA; ${ }^{2}$ Temple University, Philadelphia, PA; ${ }^{3}$ Main Line Health System Undergraduate Medical Education, Wynnewood, PA; ${ }^{4}$ Health Career Collaborative, Inc. Burlingame, CA

Statement of Significance: Higher health literacy is associated with better health outcomes. For underserved adolescents in low-income communities who are at high risk of poor health outcomes, understanding how to improve health literacy is important. Understanding a target population's health literacy allows health care providers and researchers to provide tailored interventions and better holistic patient care. In this study, we explore whether participation in the Health Career Collaborative's pathway program has an impact on the health literacy of adolescent participants.

Research Methods: Medical school mentors volunteered to conduct weekly visits to high school classrooms in traditionally underserved areas as part of the Health Career Collaborative pathway program. With the guidance of a medical student mentor, 9th and 10th grade student participants worked through interactive ER cases; and 11th grade student participants participated in project-based learning across various public health modules. After receiving IRB exemption through the Main Line Health System, this study recruited Health Career Collaborative 
student participants from Palo Alto, California; Boston, Massachusetts; and Austin, Texas. The survey consisted of thirty-three word association questions, based on established health literacy frameworks, that evaluated students' understanding of health-related terms and concepts prior to and after one academic year of participation in the HCC program. Each survey focused on language that is central in the health topics taught in the HCC curriculum.

HCC mentors administered the paper survey at the beginning of the school year to students who agreed to participate, and then again at the academic year's completion.

Data Analysis: Responses from paper surveys were entered into a database by study author (SM) and coded in a binary fashion of either correct or incorrect. Paired t tests were used to compare pre- and post-program survey responses and confidence intervals were also calculated using Excel 365 Version 2017.

Results: A total of 20 student participants completed both pre- and post-program surveys. Student participants in Grades 9-11 were 75\% female, $60 \%$ Hispanic and $10 \%$ African American. They ranged between 14 and 17 years old, and came from schools that serve low-income communities with $88 \%$ of students in East Palo Alto, California; 55\% in Boston, Massachusetts; and $77 \%$ in Austin, Texas receiving free or reduced lunch.

On average, student participants answered 18 items correctly out of 33 on the pre-program assessment, and 22 out of 33 on the post-program assessment. $95 \%$ of student participants showed improvement in their post-program assessment scores. The difference in pre- and post-program assessment scores was significant $(p=0.004)$, and student participants' score increases ranged from 1 to 6 additional correct answers.

Conclusion: When comparing the average results of the pre- and post-program assessments, there was a marked improvement in the overall number of items correct on the post test, in comparison to the pre-test. Our results suggest that over the span of the academic year, the HCC program helped improve the health literacy of its participants. While the sample size for this study was small, the results of this pilot suggest further investigation is warranted. Future studies should administer these surveys to a greater number of student participants to see if similar results are yielded.

Acknowledgment/Funding Source: Main Line Health System of Wynnewood, Pennsylvania

\section{Reference}

1. Nutbeam, D. "The evolving concept of health literacy." Social Science \& Medicine. 2008, 67:2072-2078

Poster No. ${ }^{*} \mathrm{C} 41$

Abstract No. 41

Category: Clinical

Research Focus Area: Chronic

Diseases \& Conditions

Females With Young Onset

Parkinson's Disease: A Forgotten

Demographic? An Investigational

Inquiry of Health and Social Factors

Laura Ketigian, OMS III; Kaylie Mcgivney, OMS II; Nicholas Piniella, OMS II; Rosemary Gallagher, PT, DPT, PhD; Adena Leder, DO, FAAN

New York Institute of Technology (NYIT), Old

Westbury, NY

Statement of Significance: Males are 1.5 times more likely to be diagnosed with PD than females, with prevalence favoring older adults. 
However, females diagnosed with YOPD present with symptoms, social and personal factors that differ from the typical older males with PD. Our aim is to identify potential correlations between disease characteristics and past medical, family and social history in females with YOPD. Osteopathic philosophy considers the whole person, not just the disease. In the current study, we investigate the mind, body, and spirit of women with YOPD with the goal of learning how to take a more holistic and effective approach to disease management.

Research Methods: This New York Institute of Technology College of Osteopathic Medicine (NYITCOM) Institutional Review Board exempt study recruited 39 females with a diagnosis of YOPD from the Young Women's Patient Education Virtual Forum held on May 28th, 2020. This forum provided an interactive educational platform between women worldwide with YOPD and professionals with expertise in PD. Participants who met the eligibility criteria were asked to complete a custom online survey with an incentive of a $\$ 25$ gift card to the first 50 responders. Questions included topics such as general demographics, PD course, medications, and medical, surgical, family, and social history. Topics specific to the females with YOPD were also included such as obstetric and gynecological history. Inclusion criteria: Females with diagnosis of YOPD between the ages of 21-50, stages I-IV Hoehn and Yahr scale. Exclusion criteria: Males; females with Juvenile Parkinson's Disease diagnosed at age 21 years and younger; females with Parkinson's disease diagnosed at ages 51 years and older.

Data Analysis: The survey was distributed using Research Electronic Data Capture (REDCap), a secure database. Data was de-identified for participant anonymity and exported into excel where means and percentages were calculated.

Results: Twenty six of 39 females (mean age 48.7 years; SD 5.9, range 38-62) completed the survey (66.7\% response rate). Most respondents (73.1\%) were Caucasian. Mean age at diagnosis was 41.8 years (SD 6.1, range 27-50 years). At diagnosis, the most common motor symptoms were tremor, stiffness and poor handwriting; the most common motor symptoms reported currently are stiffness, rigidity, and bradykinesia. Common non-motor symptoms include fatigue, pain/aches/tingling/ cramps, sleep problems and constipation, and neuropsychiatric symptoms including anxiety, depression, and apathy, which are similar to those at time of diagnosis. Regarding health history, $31 \%$ reported iron deficiency anemia; $0 \%$ reported cancer. Common family history includes PD, breast cancer, and heart disease. Average age of first menses was 13 years and 46\% reported having used contraceptives. Forty six percent reported worsening or new PD symptoms during menstruation, and $81 \%$ reported overall changes in libido and sexual function. Twenty one have been pregnant, of which $52 \%$ had at least one c-section. Fifty two percent had at least one miscarriage, abortion or stillbirth. Twenty seven percent reported a history of emotional and/or sexual abuse. Eighty five percent reported difficulties with sleep; on average, participants reported 7 hours of sleep before diagnosis and 5.2 hours after diagnosis. Ninety two percent take PD medications, $81 \%$ drink caffeine and $85 \%$ exercise weekly.

Conclusion: This study contributes to the knowledge of the medical and social background, as well as the manifestation of PD in a young, female population. Young females with PD present with differences in symptoms at the time of diagnosis compared with later in the disease 
process, but also present with singularly feminine manifestations of the disease, such as worsening of symptoms during menses, the number of miscarriages, and changes in libido and sexual function, suggesting possible hormonal associations. Knowledge of the unique symptoms and challenges specific to this cohort may provide insight into potential risk factors for PD in young females, improve accuracy in diagnosis, and lead to more targeted treatment, which may result in an increased quality of life. Osteopathic physicians, trained in the philosophy of addressing the whole person, are strategically positioned to manage the health and well-being of this often overlooked group. Limitations lie in our lack of racial diversity and small sample size. However this study is ongoing and our sample size will increase as we reach beyond the participants from the symposium. Future directions include collecting biomarkers such as found in saliva, and investigating temporal associations of symptoms and PD onset with medical and social history, and the inclusion of women from different racial backgrounds.

\section{Acknowledgment/Funding Source: The} researchers appreciate private funding from the Richter family for the gift card incentive supplied to the current study.

\section{References}

1. Shulman, L. M., \& Bhat, V. (2006). Gender disparities in Parkinson's disease. Expert Review of Neurotherapeutics, 6(3), 407-416. https://doi.org/10.1586/14737175.6.3.407

2. Mehanna, R., \& Jankovic, J. (2019). Young-onset Parkinson's disease: Its unique features and their impact on quality of life Parkinsonism \& Related Disorders, 65, 39-48. https://doi.org/10. [ABS]1016/j.parkreldis.2019.06.001

3. Haaxma, C. A., Bloem, B. R., Borm, G. F., Oyen, W. J. G. Leenders, K. L., Eshuis, S., ... Horstink, M. W. I. M. (2007). Gender differences in Parkinson's disease. Journal of Neurology, Neurosurgery \& Psychiatry, 78(8), 819-824. https:// doi.org/10.1136/jnnp.2006.103788

4. Calne, S. M., \& Kumar, A. (2008). Young onset Parkinson's disease. Practical management of medical issues. Parkinsonism \& Related Disorders, 14(2), 133-142. https://doi.org/10.1016/j. parkreldis.2007.07.007
Poster No. C42

Abstract No. 42

Category: Clinical

Research Focus Area: Chronic

Diseases \& Conditions

Virtual Shared Medical Appointments

for Veterans With Type II Diabetes A Resident-Driven Quality

Improvement Pilot Project

Trevor G Archibald, DO ${ }^{1}$; Marina Izzi, PharmD, PGY-2 Pharmacy Resident ${ }^{2}$; Tracy Masi, PsyD, PCMHI-ICT Post-doctoral Psychology Fellow ${ }^{3}$;

Sarai Ambert-Pompey, MD ${ }^{1}$; Amber Fisher, PharmD ${ }^{2}$

${ }^{1}$ Internal Medicine Residency, University of Washington, Boise, ID; ${ }^{2}$ Pharmacy Residency, Idaho State University, Boise, Idaho; ${ }^{3}$ Boise VA Medical Center, Boise,ID

Statement of Significance: Few interventions provide the wraparound support needed in chronic diseases such as T2DM. Shared-medicalappointments (SMAs) are a health care delivery innovation, which have proven benefit for patient access and outcomes in chronic diseases $[1,3,4]$. Inherent benefits such as collaboration, wholeperson care, and emphasis on patient co-production are central drivers of SMAs improved patient satisfaction and chronic care measures. Fundamentally SMAs promote a sociocultural exchange regarding diabetes care and lifestyle modification [4]. In this pilot project, we show that use of the VA Veteran Video Connect (VVC) video telehealth platform to deliver a shared interprofessional curriculum can produce these known beneficial impacts of SMAs.

Research Methods: Patients with Type 2 diabetes $(\mathrm{Hgb} \mathrm{A} 1 \mathrm{C}>6.5 \%)$ enrolled in VA outpatient clinics of the Boise Veteran Affairs Medical Center were recruited to undergo 4 monthly group visits utilizing a virtual telehealth curriculum created and facilitated by an interprofessional team of medicine, pharmacy, and psychology 
trainees. Seven selected patients agreed to connect to the VVC platform via personal electronic device to participate in the group intervention. We gathered pre- and post-intervention biophysical parameters, diabetes-related quality of life modified (dQOLm) scores and opinions related to intervention delivery for feasibility analysis.

At each appointment, an individual evaluation assessed for complications, co-morbidities, hypoglycemia, and need for specialist consultation. Medical record review prior to each meeting elucidated patient history of complications and co-morbidities as well as receipt of guideline-recommended screenings and therapies. A portion of each group visit was dedicated to a sequential, structured curriculum delivered by the trainee clinicians regarding diabetes pathophysiology, interprofessional care, and biopsychosocial modifications.

Disease control parameters (A1C, blood pressure, kidney function) and body weight were desired for follow-up, but elective monitoring was suspended due to outbreak of COVID-19 disease activity. Fortunately, delivery of this primarily psychosocial intervention continued as scheduled in a virtual format.

Data Analysis: Participants were asked to complete a voluntary verbal dQOLm scale using a normative Likert Scale and asked open-ended questions regarding the virtual delivery format, group experience, length/frequency of sessions, appropriateness of trainee facilitators, and suggestions for future groups.

Five patients completed the intervention and follow-up. One patient was excluded from analysis with incomplete data and one recruited patient was lost to follow-up. At least two trainees evaluated the participant responses and coded them to a numerical score for the dQOLm.
Disagreements were were settled by consensus discussion. The pre- and post-intervention dQOLm scores were compared using a paired $\mathrm{T}$ test. Narrative responses to open-ended questions were combined in a narrative synthesis reviewed by at least two researchers and resolved through consensus.

Results: Between January 2020 and May 2020 patients attended an average of three out of four planned SMAs (75\%). The average age was 70 $(+/-8.38)$ and $100 \%$ male. Statistically significant improvement in diabetes-related quality-of-life (dQOLm) was observed during the study period (mean 36.2 vs 25.8 [ $p$ value $=0.0211$ ]) signifying improved satisfaction with diabetes care and confidence in self-care abilities.

Mostly positive sentiments resulted from qualitative patient surveys regarding intervention format and perceptions of care. Narrative analysis suggests VVC technology had equal efficacy and improved convenience compared with in-person care. The virtual group intervention was also largely preferred to in-person groups, due to receipt of individualized advice, which also diffused attention. Some patients expressed hesitation regarding groups initially, but at completion they preferred a group intervention compared with individual visits.

Trainees expressed positive perceptions of the interprofessional intervention delivery, with clarity added to the scope of other professions. The provider outlook of the group experience found patients were more relaxed, which fostered an interactive and conversational style. Drawbacks included a notable investment in clinic setup by providers, while some found VVC blunted the social cues apparent during an in-person visit with amplified conversation pauses and magnification of more verbose patients. 
Conclusion: Patients with diabetes attained improved satisfaction and confidence in self-care abilities in conjunction with quality interprofessional care through this focused 4-month virtual intervention. The creation of a new clinic and delivery structure was additionally feasible within the regulatory and reimbursement framework of the Boise VA Medical Center. The quality improvement paradigm of this pilot project brought several suggestions for improvement. Many barriers to SMA implementation were surmounted by a virtual telehealth delivery system, though these were replaced by some technological issues.

While virtual SMAs are a feasible method to deliver intensive lifestyle intervention and quality care to select military Veterans through VVC, there are notable drawbacks to greater implementation. Clearly mobile telehealth platforms are not suitable for every patient, as some populations may not have access to electronic devices or internet connectivity. Fortunately, Veterans Health Administration is putting resources behind telehealth in this regard with provision of internet connected devices and monitoring equipment for veterans in certain cases. Remote evaluation via Accessing Telehealth through Local Area Stations (ATLAS) is also available.

Given the evidence provided by this small pilot study population, VVC telehealth SMAs may be a feasible method of improving diabetes care quality while promoting partnership with patients as co-producers of beneficial health outcomes.

Acknowledgment/Funding Source: Boise VA Medical Center - Center of Excellence in Primary Care Education, University of Washington, University of Idaho

\section{References}

1. Wadsworth, K, Archibald, T, Payne, E, Cleary, K, Haney, B, Hoverman, A. Shared medical appointments and patient-centered experience: a mixed methods systematic review. BMC Family Practice 20, Article number: 97 (2019). https://rdcu.be/bJmNw

2. Batalden, M, Batalden, $P$, Margolis, P, Seid, M, Armstrong, G, Opipari-Arrigan, $L$, et al. Coproduction of healthcare service. BMJ Qual Saf. 2016; 25:509-17.3.

3. Booth, A, Cantrell, A, Preston, L, Chambers, D, Goyder, E. What is the evidence for the effectiveness, appropriateness and feasibility of group clinics for patients with chronic conditions? A systematic review. Southampton: NIHR Journals Library; 2015. http://www.ncbi.nlm.nih.gov/books/NBK333454/. Accessed 9 Feb 2016

4. Edelman, D, Gierisch, JM, McDuffie, JR, Oddone, E, Williams, JW. Shared medical appointments for patients with diabetes mellitus: a systematic review. J Gen Intern Med. 2015;30:99-106.

$\star$ Poster No. ${ }^{*} \mathrm{C} 44$

Abstract No. 44

Category: Basic Science

Research Focus Area: Chronic

Diseases \& Conditions

\section{Heat Shock Protein Regulation of Intestinal Glucose Transport: Mechanism to Prevent Obesity}

Nicole Eck, OMS II'; Julia Moore ${ }^{2}$; Kristina Martinez-Guryn, $\mathrm{PhD}^{2}$; Mae J. Ciancio, $\mathrm{PhD}^{2}$ ${ }^{1}$ Midwestern University Chicago College of Osteopathic Medicine, Downers Grove, IL; ${ }^{2}$ Biomedical Sciences Program, College of Graduate Studies, Midwestern University Chicago College of Osteopathic Medicine, Downers Grove, IL

Statement of Significance: According to the World Health Organization (WHO), over 1.9 billion adults are overweight and over 650 million of those adults are obese. Obese adults are at risk for a host of health-related complications, such as type 2 diabetes and cardiovascular disease. HSP70 is a highly conserved, inherent, cytoprotective protein which maintains and structural and functional property of cells during stress. Identifying inherent mechanisms within the body that may alter nutrient uptake without changing diet will be beneficial in creating new strategies to prevent obesity in millions of people 
while following the osteopathic tenet that the body is capable of self-healing and self-regulation.

Research Methods: Male, HSP70 TG mice ( $n=5)$ and wild-type C57B1/6 (WT; n=6) mice were gavaged with $2 \mu \mathrm{Ci}$ 14C-glucose after an overnight fast. Mice were euthanized 4 hours later, plasma was collected following cardiac puncture, and intestinal mucosal scrapings (duodenum, jejunum, ileum), liver, fat (epididymal, retroperitoneal, inguinal, and mesenteric), muscle, and stool were harvested and solubilized overnight (Solvable). Scintillation fluid was added to each sample prior to determining their respective levels of radioactivity (dpms/ $\mu$ l for plasma and $\mathrm{dpms} / \mathrm{mg}$ for tissue) by scintillation counting.

Data Analysis: Data was analyzed using the Kolmogorov-Smirnov and Shapiro-Wilk tests to assess for normality, followed by a parametric $\mathrm{T}$ test for normal data or a Mann-Whitney U test for non-normal data. Significance was determined at $\mathrm{p}<0.05$ with a sample size of 6 for WT mice and 5 for HSP70 TG mice. Outliers were removed using the ROUT method of outlier detection.

Results: 14C-glucose levels were significantly elevated in the plasma, and duodenal and jejunal scrapings in the HSP70 TG mice compared with the WT controls. Ileal scrapings and stool yielded insignificant differences. Tissue uptake was greater in the liver, gonadal fat, and retroperitoneal fat tissue samples of the HSP70 TG mice compared with the WT controls. No significant differences between the genotypes were observed in the mesenteric and inguinal fat pads.

Conclusion: HSP70 TG mice demonstrated slower glucose uptake compared with the WT controls, as indicated by the significantly higher 14C-glucose levels in the plasma, duodenal and jejunal scrapings compared with the WT mice. Elevated glucose levels in the liver, gonadal and retroperitoneal fat was unanticipated, especially since previous studies
(2) demonstrated reduced adiposity in HSP70 TG mice compared with controls. It is unclear how the current findings relate to these mice having lower body weight and body fat. One explanation is that despite increased glucose uptake, there is an increase in energy expenditure, which is yet to be examined. Current studies are ongoing in the Ciancio laboratory to determine the total SGLT-1 and GLUT-2 gene expression in jejunal epithelial cells of HSP70 TG mice and whether the differential localization or activity of these two glucose transporters differs depending on genotype.

Acknowledgment/Funding Source: This project was funded by the Kenneth Suarez Summer Research Fellowship, Chicago College of Osteopathic Medicine, and the College of Graduate Studies at Midwestern University.

\section{References}

1. Chung, J. et al. HSP72 protects against obesity-induced insulin resistance. Proceedings of the National Academy of Sciences 105, 1739-1744 (2008).

2. Glawe, A. et al. 592 Continuous Epithelial Cell Heat Shock Protein 70 Expression Protects Against High Fat, Diet-Induced Obesity in Mice. Gastroenterology 146, S-111-S-112 (2014).

\section{Poster No. ${ }^{*} \mathrm{C} 46$}

Abstract No. 46

Category: Clinical

Research Focus Area: Impact of Osteopathic Manipulative Medicine (OMM) \& Osteopathic Manipulative Treatment (OMT)

The Use of Osteopathic Manipulation to Facilitate Bone Remodeling in a Postmenopausal Osteoporotic Woman Yisroel Grabie, OMS II; Nawshin (Zara) Jamil, OMS III; Naim Habib, OMS II; Joseph J. Simone, DO New York Institute of Technology (NYIT), Old Westbury, New York

Statement of Significance: In this pilot study, we investigated the efficacy of OMT in the treatment 
of osteoporosis by monitoring OSCAL and SNTX. We hypothesized that OMT could help facilitate the bone remodeling process and be used in conjunction with established therapies to maintain or increase BMD in a postmenopausal 60-year-old osteoporotic female. Whereas conventional WBE is an active process, OMT is rather passive, enabling treatment in patients with physical limitations. We used two common techniques; seated articulatory technique and pedal pump. We hypothesized that OMT would induce a significant change in OSCAL and SNTX- demonstrating a positive effect on the bone remodeling process in our sixty-year-old postmenopausal osteoporotic volunteer

Research Methods: Twelve biweekly treatments consisting of seated articulatory technique and a vigorous pedal pump were administered in an outpatient facility. Since bone deposition occurs in proportion to compressional load, the seated articulatory technique would introduce both a rotary and translatory force into the thoracic vertebrae (T1-T12). Similarly, the pedal pump would create axial compressive force and aid in lymphatic drainage throughout the lower body.

Per session, the seated articulatory technique was performed for five minutes and the pedal pump was performed for ten minutes. We used OSCAL and SNTX as measures for bone remodeling. Baseline laboratory values were obtained prior to the start of the treatment period and after the six-week treatment.

Data Analysis: Analysis of this pilot study indicates that OMT induced a significant increase in SNTX and a negligible change in serum OSCAL. We are currently in the process of replicating this study on a larger scale in order to address limitations inherent to the study's design and sample size
Results: SNTX measures were significantly increased compared with baseline, while there was no significant change in OSCAL. Prior to treatment, OSCAL and SNTX were $23 \mathrm{ng} / \mathrm{mL}$ and $4.0 \mathrm{nM} \mathrm{BCE} / \mathrm{L}$, respectively. Following treatment, OSCAL was $21 \mathrm{ng} / \mathrm{mL}$ and SNTX was $15.2 \mathrm{nM} \mathrm{BCE} / \mathrm{L}$.

Conclusion: Increased SNTX is consistent with bone remodeling. We hope to investigate the lack of OSCAL change in our follow up study. We believe it is possible that OMT could provide synergism in the bone remodeling process. We hope to repeat this protocol with a larger cohort and measure osteoclastic and osteoblastic activity via additional markers of bone remodeling such as PTH, vitamin D, calcitonin, and confirmative densitometry. The results of this study demonstrate the need for collaboration in the osteopathic community to facilitate both the investigation and application of "passive mechanotransduction" to replicate the remodeling benefits associated with weight-bearing activities.

Poster No. *C51

Abstract No. 51

Category: Clinical

Research Focus Area: Osteopathic Philosophy

Changes in Heart Rate, Activity Level, and Heart Rate Variability in Cardiac Patients With Implantable Loop Recorders During the COVID-19 Pandemic Emily Dries, OMS III; Alyssa A. Curcio, OMS II; Nicole A. Falus, OMS II; Todd J. Cohen, MD New York Institute of Technology College of Osteopathic Medicine, Old Westbury, New York

Statement of Significance: This natural experiment can elucidate the physiological changes 
patients experience in the presence of a national stressor. Osteopathic physicians can use these findings to adapt their holistic treatment of their patients, knowing the potential mental and physiologic changes that can arise during a national stressor. This information can better inform physician treatment and counseling plans to improve patient health and well-being.

Research Methods: The study "Diagnostic and Therapeutic Outcomes from the Long Island Heart Rhythm Center (LIHRC)", a registry study which retrospectively analyzes LIHRC clinical data, received IRB approval from New York Institute of Technology (BHS-145).

Cardiac patients of the LIHRC with Medtronic Reveal LINQ TM implantable loop recorders (ILRs) were considered for this study. ILRs are cardiac monitoring devices placed under the chest wall that serve as electrocardiograms, continuously monitoring heart rate and activity. ILRs have previously been shown to be safe and accurate monitoring devices.

Patients were included if their ILR records were available for the study dates of interest: 60-days before and 60-days after the declaration of a national emergency on 3/13/20. The "Pre-National Emergency" period runs from January 13 through March 12, 2020. The "Post-National Emergency" period runs from March 13 through May 12, 2020. Exclusion criteria included incomplete transmission history or missing data points from transmission history. Data was further excluded from each measure if the readings were out of range of the ILR measurements. This included heart rate below $60 \mathrm{bpm}$ or above $120 \mathrm{bpm}$, and heart rate variability below $40 \mathrm{~ms}$ or above $200 \mathrm{~ms}$.
Included patients' ILR records were studied for the dates of interest. Daily data on day-time heart rate, patient activity, and heart rate variability was extracted from ILR reports using Web Plot Digitizer and compiled for the dates of interest.

Data Analysis: Each participant's day-time heart rate, activity level, and heart rate variability were averaged for the "Pre-National Emergency" and "Post-National Emergency" periods. Patients served as their own "controls", and paired t tests were conducted for each measure to assess for any significant changes between the two time periods. Statistical analyses were performed using JASP statistical software. P-values $<0.05$ were considered statistically significant. Descriptive statistics were performed when appropriate.

Results: A total of 67 patients form the LIHRC were found to have Medtronic Reveal LINQ TM ILRs. Of these patients, 54 had reports within the study period, and of those, 36 had data for each day within the study period. Patients were further excluded from the activity measurements for missing data $(\mathrm{n}=1)$, from the heart rate measurements for missing data $(n=1)$ and data out of range of measurement $(\mathrm{n}=18)$, and from heart rate variability measurements for missing data $(n=3)$ and data out of range of measurement $(n=4)$.

The ages of included patients ranged from 24.6 years to 94.2 years, with the average being 66.6 years. The age of implant of the ILR ranged from 0.8 years to 3.5 years, with the average age of implant being 1.7 years. $20 \%$ of the included patients were male and $80 \%$ were female.

There was no significant difference between the average "Pre-Emergency" day-time heart rate $(82.2 \mathrm{bpm})$ and the average "Post-Emergency" 
day-time heart rate $(81.0 \mathrm{bpm})$ for each of the 17 included patients $(\mathrm{p}=0.112)$.

The "Pre-Emergency" average activity of the 35 included patients was 2.1 hours, and the "Post-Emergency" average was 1.8 hours. There was a significant decrease in the average activity time per patient between "Pre-Emergency" and "Post-Emergency" ( $\mathrm{p}<0.001)$.

The average heart rate variability during the "Pre-Emergency" period for the 29 included patients was $107.1 \mathrm{~ms}$, and the "Post-Emergency" period was $108.0 \mathrm{~ms}$. There was no significant difference between these averages $(p=0.514)$.

Conclusion: Cardiac patients with ILRs were found to have statistically significantly lower activity levels during the 60 days post-declaration of a National Emergency for the COVID-19 pandemic, as compared with the 60 days predeclaration. This finding is likely explained by the "shelter in place" recommendations during the COVID-19 pandemic and the closing of many businesses in the state of New York.

While there was no significant difference in patient heart rate, the authors hypothesize this could be because any increase in heart rate from the stress of the pandemic was offset by a decrease in heart rate from reduced activity.

Osteopathic physicians can use this knowledge to create treatment plans with their patients' lower activity levels in mind. For example, physicians can counsel patients on how to maintain healthy diets and exercise in the comforts of their own home, while still practicing safety and social distancing.

Limitations of this study include its retrospective, observational nature, small sample size and the proportion of excluded patients. It is impossible to determine if the changes measured were due to the COVID-19 pandemic or external factors. Additional research should confirm these findings in a larger population by incorporating new ILR patient data via a multi-center approach. Future spikes of COVID-19 may provide an opportunity for further ILR analysis and confirmation of these findings.

\section{References}

1. Ranjbar, F, Akbarzadeh, F, Kazemi, B, Ranjbar, A, Sharifi, Namin S, Sadeghi-Bazargani, H. Increased Likelihood of Arrhythmic Events Associated with Increased Anxiety in Patients with Implanted Cardiac Defibrillators after the Ahar-Varzaghan Earthquake in East Azerbaijan, 2012. Bull Emerg Trauma 2016;4(4):202-210.

2. Loop Recorder Implantation. Johns Hopkins Medicine. https:// www.hopkinsmedicine.org/health/treatment tests-and-therapies/ loop-recorder-implantation. Accessed June 10, 2020.

3. Maines, M, Zorzi, A, Tomasi, G, et al. Clinical impact, safety, and accuracy of the remotely monitored implantable loop recorder Medtronic Reveal LINQTM. Europace. 2018;20(6):1050-1057. doi:10.1093/europace/eux187

$\star$ Poster No. *C52

Abstract No. 52

Category: Basic Science

Research Focus Area: Chronic

Diseases \& Conditions

\section{Brain and Skull Interactions in Polish Crested Chickens as a Model for Hydrocephalus \\ Scott Matthew Landman, OMS II ${ }^{1}$; Yekaterina Okouneva, OMS II ${ }^{1}$; Paul M. Gignac, $\mathrm{PhD}^{2}$; Akinobou Watanabe, $\mathrm{PhD}^{3}$ \\ ${ }^{1}$ New York Institute of Technology (NYIT), Old Westbury, NY; ${ }^{2}$ Department of Anatomy and Cell Biology, Oklahoma State University Center for Health Sciences, Tulsa, OK; ${ }^{3}$ Department of Anatomy, New York Institute of Technology (NYIT), Old Westbury, NY}

Statement of Significance: Hydrocephalus occurs 1 in $\sim 1500$ births [2]. However, research remains limited due to lack of readily available strains that exhibit hydrocephalus. This study 
introduces a new model organism that exemplifies the unique phenotypes associated with hydrocephalus- one that is accessible and easily maintained. Elucidating how cerebral herniation and skull morphology are connected also has relevance to acquiring a nuanced perspective on how seemingly distinct organs interact with one another. With a better understanding of hydrocephalus and how its phenotypic symptoms are coupled, Osteopathic physicians may become better equipped to symptomatically treat patients in conjunction with more traditional treatment modalities such as shunt placement.

Research Methods: In this proposed study, we introduce a new model organism, the Polish crested chicken (PCC) a breed of domesticated chickens that possess a prominent cranial protuberance and a unique brain morphology; an inflated cerebrum that herniates into the superior aspect of the cranial vault during development ${ }^{3}$. Previous studies have provided evidence that hydrocephalus may be the driver of their unique brain and craniofacial anatomy [4]. Despite their highly divergent cranial and brain morphologies, typical domestic chickens (TDC) and PCCs are closely related lineages, forming a compelling comparative system to examine how the new brain and skull morphologies arise. Here, we employed high-resolution $\mathrm{x}$-ray micro-CT imaging on 21 specimens of PCCs and 9 specimens of TDC to create 3-D models of their brain morphology and skull. We then used a highdensity 3-D geometric morphometric approach to quantify the shape of the skull and brain and their individual developmental and functional regions.

Data Analysis: We measure the pattern and strength of anatomical interactions within and between the brain and the skull via covariation analysis. Using the R statistical programming language, we performed a suite of multivariate statistical analyses, including two-block partial least squares [5] analysis, to visualize, model, and test the pattern and magnitude of the covariation between individual skull and brain regions, as well as between the overall skull and brain shapes.

Results: Based on the analysis of TDC, the skull shape and brain shape are highly correlated with each other ( $r-P L S>0.8$ ). The brain, which develops earlier than the skull, largely influences the shape of the surrounding cranial vault, and interestingly, the face, specifically in the narrowness of the beak and relative size of the external naris. When we analyzed the correlation between each brain region to the overall skull shape, the cerebrum and optic lobes had the highest correlation with skull shape ( $r-P L S \geq 0.9 ; \mathrm{P}<0.05$ ). We anticipate similar findings in PCCs, supporting our hypothesis that the cerebrum and optic lobes have the greatest influence on skull shape, particularly in the skull roof and beak shape. In addition, the expanded cranial vault of PCCs is expected to have emerged without breaking the existing strong correlation between the skull roof and the beak observed in typical chickens.

Conclusion: High-density shape analysis reveals the heterogeneous influence of the brain on skull morphology, where certain brain and cranial regions show strong interactions. For instance, this study demonstrates that brain shape is correlated with not only the shape of the surrounding cranial vault but also the facial anatomy (beak and naris). This outcome supports a previous developmental study that shows key molecular signaling from the forebrain contributes to the beak formation in birds [6]. Therefore, major 
changes in brain morphology during development have amplified effects on the cranial vault and the face, but not on other parts of the skull. In addition, our study shows that Polish crested chickens have enormous potential to be a model system for hydrocephalus and for gaining a more holistic understanding of how new phenotypes can emerge through interactions across multiple organs and tissue types. However, because previous studies on PCC is very limited, confirmation that hydrocephalus is the key mechanism underlying their cerebral herniation and cranial expansion in PCCs is needed using modern techniques, such as antibody staining, volumetric comparisons of brain and ventricular volume during development, and measurement of CSF content.

Acknowledgment/Funding Source: Skulls Unlimited and Doug Warner at Charles River Laboratory provided PCC and TDC specimens respectively. PCCs were scanned at MICRO Facility (Fayetteville, AR) \& TDC specimens were scanned at Microscopy \& Imaging Facility at the American Museum of Natural History. Summer Research Program from NYITCOM, National Science Foundation (DEB-1406849 to A.W.; BCS-1725925 to P.M.G.), Jurassic Foundation (to A.W.), and Society of Vertebrate Paleontology funded the data collection and this study.

\section{References}

1. Rekate, H.L. (2008). Cerebrospinal Fluid Research 5, 1-Ip.

2. Schurr, P.H., Mclaurin, R.L., Ingraham, F.D. (1953). Journal of Neurosurgery $10,515-525$.

3. Yoshimura, K. Kinoshita, K., Mizutani, M., Matsuda, Y., Saito, N. (2012). Journal of Experimental Zoology Part B 318, 1-8. 10.1002/jez.b.22464.

4. Klatt, B. (1911). Zur Frage der Hydrocephalie beiden Haubenhühnern. Sitzungsbericht der Gesellschaft Naturforschender Freunde zu Berlin, No. 2, 75-84 + plate III.

5. Rohlf, F.J., Corti, M. (2000). Systematic Biology 49, 740-753.

6. Marcucio, R.S., Cordero, D.R., Hu, D., Helms, J.A. (2005). Developmental Biology 284, 48-61.
Poster No. *C54

Abstract No. 54

Category: Clinical

Research Focus Area:

Musculoskeletal Injuries and

Prevention

\section{Long-Term Self-Reported Functional Outcomes of Clavicle Fractures} Treated Nonoperatively vs Operatively Bryce Beatty, OMS $\|^{1}$; Kellie Gaura, OMS $\|^{2}$; Hailey Boyd, OMS II²; Edie Sperling, PT, DPT, OCS ${ }^{2}$; Johannie Spaan, $\mathrm{PhD}^{3}$; Robyn Dreibelbis, DO, FACOFP $^{4}$

${ }^{1}$ Western University of Health Sciences COMP, Lebanon, OR; ${ }^{2}$ Department of Medical Anatomical Sciences, Western University of Health Sciences COMP-NW, Lebanon, OR; ${ }^{3}$ Department of Basic Medical Sciences, Western University of Health Sciences COMP-NW, Lebanon, OR; ${ }^{4}$ Department of Family Medicine, Western University of Health Sciences COMP-NW, Lebanon, OR

Statement of Significance: Clavicle fracture treatment is well researched although it remains highly debated whether to treat the injury nonoperatively or operatively. Historically, nonoperative treatment has been the standard of care, however recent studies have found higher rates of impaired biomechanics with nonoperative management of displaced and shortened fractures (Luo et al., 2015; Waldmann et al., 2018). There is limited research that assesses functionality and biomechanics on a long-term basis as current research has focused on assessing functionality and biomechanics until fracture union in a retrospective manner without patient-reported outcomes (Luo et al., 2015). Therefore, in this study we addressed the gap in research in patient reported outcomes.

Research Methods: Current 2nd and 3rd-year students at Western University of Health Sciences in the colleges of Osteopathic Medicine, Pharmacy, Graduate Nursing, Veterinary Medicine, Optometry, Podiatric Medicine and 
Biomedical Sciences were invited to take a 10 minute online survey with varying number of questions dependent upon their history of having a prior clavicle fracture and whether they were treated nonoperatively or operatively. The data was analyzed both qualitatively and quantitatively to evaluate self-reported treatment protocol and functional outcomes including perceived strength, range of motion, and pain/symptoms. IRB approval number: X20/IRB/038.

Data Analysis: Generalized linear models with a binomial distribution and logit link function was used for each self-reported functional outcome and symptom (binary dependent variables) in patients who underwent surgery for a clavicle fracture vs those treated non surgically. The number of years since the most recent clavicle fracture was included in each model to account for the time differences of functional outcomes and symptoms between each patient. Visual inspection of model assumptions revealed no violation of assumptions. The model coefficients were back transformed and interpreted as odds ratios. Descriptive statistics used in this study was expressed as percentages. The statistical program $\mathrm{R}$ was used for all data analysis.

Results: 38 respondents completed an online survey. The average age of participants was 27.5 years. Average time since fracture was 11.4 years with a SD of 8.7 . Of the 38 respondents, 14 had surgery (37\%) and the other 24 $(63 \%)$ did not. The odds of perceiving upper extremity strength difference was reduced by $90 \%$ if the patient had surgery $(\mathrm{p}=0.07)$, while accounting for a $22 \%$ decrease in perceived strength difference for every year after the patient's most recent fracture $(\mathrm{p}=0.03)$. Although not statistically significant, odds of decreased range of motion on affected side was reduced by $66 \%(\mathrm{p}=0.38)$ and odds of currently having pain in the clavicle, shoulder, or scapula region was reduced by $58 \%(\mathrm{p}=0.39)$ if the patient had surgery when accounting for number of years since fracture. The most common movement that elicited pain was reaching overhead for objects. Patients who had surgery were 3 times more likely to receive physical therapy $>3$ times compared with those that did not have surgery $(p=0.02)$.

Conclusion: This data suggests that patients who had surgery have better perceived equality of strength between the fractured and non-fractured upper extremity. It was not found that there was a perceived difference in range of motion between the two upper extremities. Interestingly, patients who had surgery received more physical therapy compared with non-surgical patients. Our research was solely focused on patients perceived functional outcomes, but further research should be performed to determine if subjective strength can be shown objectively in a clinical setting for those who were treated surgically compared with those who were treated nonoperatively. A limitation of this study was the small sample size. Future research should be performed and include a larger sample size so that the results may be generalized to a greater population.

\section{References}

1. Bae, D., Shah, A., Kalish, L., Kwon, J., Waters, P. (2013). Shoulder motion, strength, and functional outcomes in children with established malunion of the clavicle. Journal of pediatric orthopaedics. 33(5):544-550. doi: 10.1097/ BPO.0b013e3182857d9e

2. Luo, T. D., Ashraf, A., Larson, A. N., Stans, A. A., Shaughnessy, W. J., \& McIntosh, A. L. (2015). Complications in the treatment of adolescent clavicle fractures. Orthopedics, 38(4), e287-e291. doi:10.3928/01477447-20150402-56

3. Waldmann, S., Benninger, E., \& Meier, C. (2018). Nonoperative Treatment of Midshaft Clavicle Fractures in Adults. The open orthopaedics journal, 12, 1-6. 
Poster No. *C55

Abstract No. 55

Category: Clinical

Research Focus Area: Osteopathic Philosophy

\section{Cardiovascular Clearance in}

\section{Collegiate Athletes}

Matthew Tarrash, OMS II; Emily Dries, OMS III; Matthew Heller, DO; Hallie Zwibel, DO, MPH;

Todd J. Cohen, MD

New York Institute of Technology (NYIT), Old

Westbury, NY

Statement of Significance: Current NCAA guidelines for cardiac clearance require completion of a standardized pre-participation physical examination (PPE). A 2019 study published in the Journal of the American Heart Association (AHA) found the NCAA-recommended PPE performed poorly compared with electrocardiograms (ECGs) for cardiovascular screening in high school athletes. New York Institute of Technology (NYIT), an NCAA Division II program, has access to both a dedicated Sports Medicine program and a cardiology clinic. This study of an interdisciplinary medical team can serve as a model for other collegiate athletic programs and osteopathic medical schools, and can help physicians improve cardiac screening protocols to ensure athlete health and safety.

Research Methods: This study included "Diagnostic and Therapeutic Outcomes from the Long Island Heart Rhythm Center (LIHRC)" a registry study which retrospectively analyzes clinical data from that center. The study received IRB approval from New York Institute of Technology (BHS-145).

All NYIT athletes were initially screened by NYIT Sports Medicine physicians using the Pre-participation Screening Monograph, 4th edition (PPE4), and given an ECG for their first visit. Patients were then referred to the LIHRC for cardiac workup and/or clearance if cardiac-related symptoms and/or abnormal ECG were present or if additional positive findings on the PPE4 were found. This was completed in order for athletes to continue participation in their respective sport at the collegiate level.

This retrospective study included athletes who (1) were members of an NYIT collegiate sports team and (2) presented with cardiac-related symptoms (pre-syncope, shortness of breath, chest discomfort and palpitations) requiring an ECG. Exclusion criteria consisted of incomplete charting or records.

For included patients, a comprehensive chart review was performed to identify patient symptoms, testing and results. Testing included ECGs, trans-thoracic echocardiograms (TTE), exercise stress tests, exercise stress echocardiograms, tilt table tests, Holter monitoring, implantable loop recorder monitoring, and electrophysiology (EP) studies.

Data Analysis: Patient medical records were analyzed for presenting symptoms, diagnostic tests, testing results, and treatment plans. Data on these measures was compiled and descriptive statistics were calculated when appropriate.

Results: Seven athletes (men=4, women=3), presented with cardiac-related symptoms including shortness of breath $(\mathrm{n}=5,71.4 \%)$, palpitations $(\mathrm{n}=3,42.9 \%)$, pre-syncope $(\mathrm{n}=4$, $57.1 \%)$ and chest discomfort $(n=2,28.6 \%)$. None patients had a family history of cardiovascular disease or abnormal findings on the physical examination.

All patients were given ECGs and had abnormal findings, including rSr' patterns, LVH by voltage, 
and early $\mathbf{J}$ point elevations (two of which had suggestions of possible pericarditis).

$100 \%$ of patients $(n=7)$ were given TTE's with $85.7 \%$ having normal readings, and $85.7 \%(n=6)$ were given exercise stress tests, with $100 \%$ having normal readings.

Patients with one cardiac complaint and normal TTE and exercise stress test findings $(n=2)$ were cleared for participation. The patient with one cardiac complaint (shortness of breath) and an abnormal TTE $(n=1)$ was given an exercise stress echocardiogram. Patients with one cardiac complaint $(n=3)$ were all ultimately cleared for participation.

Patients with multiple cardiac complaints $(n=4)$ needed further testing in $75 \%(n=3)$ of cases. Patients with palpitations and pre-syncope accounted for all patients given a tilt-table test, Holter monitor, implantable loop recorder, and an EP study. Two patients had multiple of these tests and were both cleared for sports participation. One patient with continued pre-syncope and palpitations did not follow through on recommended tests and treatments, and has not been cleared for participation.

Conclusion: Using NYIT's cardiac screening guidelines in conjunction with a board-certified cardiologist, methods for clearance of seven college athletes presenting with cardiac symptoms were analyzed.

Results showed a work-up progression that took both the presenting symptoms of the patient and the ECG interpretation into consideration.

This study shows that patients with only one cardiac complaint and an abnormal ECG appear less likely to require extensive cardiac testing. Patients with two or more complaints $(n=4)$, specifically presyncope and palpitations, were more likely to require extensive cardiac testing $(n=3,75 \%)$.
This study demonstrates the importance of having a cardiologist available to work closely with a sports medicine team in evaluating and clearing collegiate athletes. Access to advanced cardiac testing is helpful in determining if athletes can continue to participate in competitive sports. The presence of cardiology and sports medicine specialists in the same clinic allows for increased communication and continuity of care, which can help improve patient experience and outcomes.

Limitations of this study include small sample size and the authors hope to gain additional experience through the continued screening of NCAA athletes.

Acknowledgment/Funding Source: We would like to acknowledge NYIT athletics. We have no funding sources to disclose.

\section{References}

1. Harmon, Kimberly G., et al "Incidence, Cause, and Comparative Frequency of Sudden Cardiac Death in National Collegiate Athletic Association Athletes." Circulation, vol. 132, no. 1, 2015 pp. 10-19, doi:10.1161/circulationaha.115.015431.

2. Wasfy, Meagan M., et al "Sudden Cardiac Death in Athletes." Methodist DeBakey Cardiovascular Journal, vol. 12, no. 2, 2016 pp. 76-80, doi:10.14797/mdcj-12-2-76.

3. Williams, Elizabeth A., et al "Performance of the American Heart Association (AHA) 14-Point Evaluation Vs Electrocardiography for the Cardiovascular Screening of High School Athletes: A Prospective Study." Journal of the American Heart Association, vol. 8, no. 14, 16 July 2019, doi:10.1161/ jaha.119.012235.

Poster No. ${ }^{*} \mathrm{C} 57$

Abstract No. 57

Category: Health Services

Research Focus Area: Osteopathic Philosophy

\section{Stratification on the Opinions of Medical Malpractice}

Kassem Michael Makki, OMS IV; Harrison

R. Wermuth, OMS IV; Kim Pfotenhauer, DO, BC-ADM

Michigan State University College of Osteopathic

Medicine, East Lansing, MI 
Statement of Significance: Medical malpractice has a significant impact on the careers of current and future osteopathic physicians. According to the AMA, 1 in 3 physicians will be sued by the age of 55. With the rise of initiatives to increase accountability and patient safety, medical malpractice is becoming more intertwined in medical school education as well as being taught in law school curriculums.

Research Methods: After IRB approval and informed consent, an anonymous survey was distributed that tested the professional's foundational knowledge of basic medical malpractice as well as presented unique case scenarios to analyze their application of medical malpractice and the professional's attitudes and beliefs on malpractice. To obtain a large cohort and research statistical significance the anonymous link was sent out via email to professional societies, reaching out directly to various law firms, posting on social media, and reaching out directly to various residency programs, hospital networks, and private physician practices. The data was collected between February 2020-July 2020 via a Qualtrics survey.

Data Analysis: The collected data was analyzed by the percentage of professional's answers in each cohort as well as an overall percentage of answers for each question. Additionally, crosstabulations for the various questions were used to extrapolate interrelated data with various cohorts. The data was arranged in various statistical applications of bar graphs, linear regression models, pie charts, and tables to display and analyze the variance of responses between the different cohorts. The total survey was composed of 176 respondents, made up of 63 males, 111 females, and 2 individuals who did not wish to disclose. Our cohorts were com- posed of 31 law students, 29 attorneys, 47 medical students, 38 residents and 31 attending physicians.

Results from the survey displayed that the various cohorts chose the same answers for the majority of the questions. However, there were several apparent stratifications in several key aspects of medical malpractice. Analysis showed that the majority of all cohorts had the same perceptions and thoughts on the definitions of medical malpractice, the importance of apologizing to patients as well as having open communication. The apparent stratification between the cohorts was the variance in responses on the most likely cause of medical malpractice, the first step to take after being named in an allegation of medical malpractice, as well as, the most important component of professional liability insurance.

Results: Analysis showed, $73 \%$ of the resident cohort and $53 \%$ of the medical student cohort thought that the number one cause of medical malpractice is an inattentive follow-up while $73 \%$ of attending physicians believed the number one cause of medical malpractice was misdiagnoses. In regard to the other professional cohorts of attorneys and law students, $62 \%$ of attorneys believe the number one cause of malpractice was a surgical error while $32 \%$ of law students believed that the degree of patient injury is the number one cause of malpractice. Based on indemnity payouts from malpractice insurance providers, the number one cited cause of medical malpractice was misdiagnosis; the majority of the attending physician cohort selected misdiagnosis as their number one answer choice at $73 \%$. The majority of respondents of the survey, $76 \%$ of total respondents, believed the two major components to choosing professional liability insurance were the length of coverage and policy limits. 
However, there was a striking difference in regards to the most important aspects of professional liability insurance with the majority of medical students (52\%), law students (55\%) and attorneys ( $82 \%$ ) believing the most important factor was policy limits while resident physicians $(36 \%)$ and attending physicians $(60 \%)$ believing the most important factor was the length of coverage.

Conclusion: In regards to the most important step after being notified they are being sued for medical malpractice, the survey found $97 \%$ of attending physicians, $89 \%$ of attorneys, $76 \%$ of law students and $60 \%$ of medical students believe the most important first is informing your insurance carrier. Surprisingly analysis showed only $32 \%$ of residents believed that the most important first step after being notified that they were named in a medical malpractice allegation of informing the insurance carrier, while most of the majority of the resident cohort (46\%) believed that the first step is to apologize to the patient. many of the answers of the survey between the cohorts having a similar responses, there were still key areas of medical malpractice that showed a great divergence of opinion. This survey can offer insight into where more education is needed and at which stage of professional development. Cross-tabulation analysis between different questions additionally displayed that the time spent at each professional institution within each cohort did not have a relationship in regard to several answer choices on the topics of malpractice. This research can aid creating risk mitigation education to reduce indemnity payouts as well as increase patient safety. It stresses the need for consistent, early education in medical malpractice in osteopathic curriculum. Finally, the results highlight the importance of interprofessional opportunities for discussions into medical malpractice.
Poster No. C58

Abstract No. 58

Category: Clinical

Research Focus Area: Impact of

Osteopathic Manipulative Medicine (OMM) \& Osteopathic Manipulative Treatment (OMT)

AOA Grant Number: 2031815722

The Efficacy of Facial Effleurage, a

\section{Lymphatic Pump Technique, on}

Immune Response and Symptoms in

Acute Rhinosinusitis: A Randomized, Controlled Study

Chelsea Weidman, $\mathrm{MS}^{1}$; Monica Boyd, OMS IV 2 ; Elizabeth Pepper, OMS IV²; Katrina Hsieh, OMS IV²; Derek T. Kunda, OMS IV²; Kyle S. Burke, OMS IV; Mario Carrasco, OMS IV²; Oshea Escamilla, DO ${ }^{3}$; Ning Cheng, MS, $\mathrm{PhD}^{4}$; Jillian $\mathrm{H}$. Bradley, $\mathrm{PhD}^{2}$ ${ }^{1}$ Edward Via College of Osteopathic Medicine (South Carolina), Spartanburg, SC; ${ }^{2}$ Department of Microbiology and Immunology, Edward Via College of Osteopathic Medicine - Carolinas Campus, Spartanburg, SC; ${ }^{3}$ Department of Osteopathic Manipulative Medicine (OMM), The Edward Via College of Osteopathic Medicine - Carolinas Campus \& Palmetto Proactive Healthcare, Spartanburg, SC; ${ }^{4}$ Department of Biomedical Affairs and Research, Edward Via College of Osteopathic Medicine - Auburn Campus, Auburn, AL

Statement of Significance: There are limited data on lymphatic pump techniques in ill people and almost no data on OMT in RS patients in the literature. The application of OMT as a first line of treatment can reduce patients' symptoms and may reduce antibiotic overprescription. Elucidating the immunological mechanism of action of lymphatic pump techniques in RS patients and quantifying the effect of OMT on symptoms will provide quantitative evidence for more widespread use of lymphatic pump techniques.

Research Methods: This was a single-session, randomized, controlled clinical study comparing 
a standardized lymphatic pump technique protocol, a sham treatment, and standard-of-care antibiotics. The Edward Via College of Osteopathic Medicine Institutional Review Board approved all study protocols. Participants between 18 and 69 years old were recruited from a rural health clinic in Spartanburg, South Carolina. Participants were diagnosed with acute RS $(n=78)$ or as otherwise healthy with no RS symptoms $(n=60)$. Participants were randomized into the following treatment groups: no treatment (healthy participants only), the lymphatic pump protocol, the sham treatment, or antibiotics (RS participants only). The lymphatic pump protocol involved five osteopathic techniques: thoracic inlet release, suboccipital release, Galbreath's maneuver, auricular drainage, and facial effleurage. The sham treatment mimicked the same hand placements and movements as the lymphatic pump techniques, but the provider did not apply any pressure. Participants filled out a validated symptom questionnaire and provided peripheral blood and nasal mucus samples before, 1 hour after, and 7-10 days after treatment was initiated. Nasal swabs were collected before and 7-10 days after treatment. If prescribed, antibiotics were started on the same day as treatment. Physicians at the clinic determined the most appropriate standard-of-care antibiotics for each patient, most commonly amoxicillin \pm clavulanate.

Data Analysis: Symptoms and their severity were tracked using the Sinonasal Outcome Test-20 (SNOT-20), a validated questionnaire ranking 20 symptoms associated with RS from 0 (none) to 5 (worst). Values were summed to generate the total symptom score (TSS) out of a possible 100 points. Nasal bacteria were cultured and identified using biochemical assays and gram staining. Results were used to determine if RS was bacterial. Two blood samples were obtained at each time point: one was centrifuged to isolate the serum, and the other was treated to obtain the white blood cells (WBCs). Serum was analyzed via ELISA to obtain immunoglobulin E ( $\operatorname{IgE}$ ), immunoglobulin $\mathrm{G}$ ( $\mathrm{IgG}$ ), immunoglobulin $\mathrm{M}$ (IgM), tumor necrosis factor alpha (TNF $\alpha)$, and interleukin 6 (IL-6) concentrations. WBC count was determined using a hemocytometer and WBC populations were quantified via flow cytometry. Concentrations of TNF $\alpha$ and various interleukins in nasal mucus samples were analyzed using a cytometric bead array via flow cytometer.

Power analysis showed that this study has $80.0 \%$ of power to detect an effect size of 16.38, with an effect size of 28. A two-tailed Fisher's Exact Test was used to compare demographic information between healthy and RS participants. An ANOVA analysis was used to compare the mean TSS of different groups. Student's t tests were used to determine all other significant differences. Significance was determined as a p-value $<0.05$. Data analysis and graphing were performed using Prism 8.0.2 (GraphPad).

Results: All data are reported as differences between baseline and 1-hour or 7-10 days posttreatment. RS participants reported significantly improved symptoms 1 hour after lymphatic pump protocol compared with RS participants who were prescribed but had not yet started antibiotics $(-15.4 \pm 8.3$ vs $-6.0 \pm 5.5, \mathrm{p}=0.0001)$. RS participants who received sham treatment also reported reduced symptoms, but the difference was not significant $(-12.3 \pm 15.2$ vs $-6.0 \pm 5.5, \mathrm{p}=0.11)$. RS participants had significantly higher serum IgM levels 1 hour after lymphatic pump treatment compared with healthy controls $(32.5 \pm 100.7$ vs -164.0 $\pm 390.4 \mathrm{mg} / \mathrm{dL}, \mathrm{p}=0.04)$. RS participants who received antibiotics and lymphatic pump treatment had significantly lower serum IL-6 levels 7-10 days after treatment compared with those who just received lymphatic pump treatment $(-13.0$ 
\pm 18.2 vs $1.6 \pm 9.8 \mathrm{pg} / \mathrm{mL}, \mathrm{p}=0.007)$. RS participants had significantly elevated serum TNF $\alpha$ levels 1 hour after lymphatic pump treatment compared with those who received sham treatment $(52.0 \pm 135.5$ vs $-63.4 \pm 132.5 \mathrm{pg} / \mathrm{mL}, \mathrm{p}=0.01)$ and those who received (but had not begun) antibiotics $(52.0 \pm 135.5$ vs $-36.3 \pm 155.5 \mathrm{pg} / \mathrm{mL}, \mathrm{p}=$ $0.045)$. Serum TNF $\alpha$ remained significantly elevated 7-10 days after lymphatic pump protocol in RS participants compared with healthy controls $(30.8 \pm 92.5$ vs $-56.3 \pm 130.1 \mathrm{pg} / \mathrm{mL}, \mathrm{p}=0.026)$.

Conclusion: Our goal was to capture and objectively measure if lymphatic pump OMT moves lymph and improves RS symptoms, which has been anecdotally shown before. Excitingly, we saw immune changes after OMT but not sham treatment, verifying the presumed mechanism of action. These beneficial effects continued for at least one week after a single OMT treatment. Importantly, participants' symptoms improve quickly after OMT, but not significantly with sham treatment. Taken together, lymphatic pump OMTs mobilize lymph within an hour, which quickly alleviates RS symptoms after a single treatment. One OMT session can improve patients' quality of life by reducing RS symptoms almost immediately and promote their healing for at least one week. Using lymphatic pump techniques, such as facial effleurage, as a first line treatment for acute RS will not only help the patient quickly feel better, but it may also reduce the use of unnecessary antibiotics. Limitations include a small sample size per treatment group and limited diversity in participants. Further studies in a larger population and with multiple OMT sessions are needed to determine how lymphatic pump OMT can best benefit RS patients.

Acknowledgment/Funding Source: We would like to thank Palmetto Proactive Healthcare for allowing us to recruit patients at their office and have their medical assistants aid in patient sample collection. This research was funded by the American Osteopathic Association (Grant \#2031815722). Collection of the pilot data was funded by a research grant through the Edward Via College of Osteopathic Medicine's Research Eureka Accelerator Program (REAP).

\section{Poster No. ${ }^{*} \mathrm{C} 60$}

Abstract No. 60

Category: Basic Science

Research Focus Area: Osteopathic Philosophy

\section{Hand Model Utility for Osteopathic} Pelvic Diagnosis in a Remote Learning Setting

Brandon Jared Burg, OMS III, AMS ${ }^{1}$; Laura A. Ketigian, OMS III, $\mathrm{AMS}^{2}$; Sheldon C. Yao, DO, $\mathrm{FAAO}^{3}$

${ }^{1}$ New York Institute of Technology (NYIT), Old Westbury, NY; ${ }^{2}$ Department of Osteopathic Manipulative Medicine, New York Institute of Technology (NYIT), Old Westbury, NY; ${ }^{3}$ Chair, Department of Osteopathic Manipulative Medicine, New York Institute of Technology (NYIT), Old Westbury, NY

Statement of Significance: Online learning challenges osteopathic medical education (OME) due to the lack of hands-on palpation. The biomechanical model of manipulation focuses on structure and function, requiring an anatomical and spatial understanding of the human body's innate motion. Efficient and effective research is required to edify students during these unprecedented times of remote learning due to the COVID-19 pandemic. Hand models offer students a means to simulate the biomechanics of the human body for proper diagnosis of somatic dysfunctions (SD). There is a gap in the literature describing the use of hand models in OME. This study aims to investigate the usefulness of hand models for learning pelvic SD diagnosis 
Research Methods: Due to the COVID-19 pandemic, first year NYITCOM students from the New York and Arkansas campuses were limited to remote learning of pelvic diagnosis. Students received an instructional online PowerPoint containing pictures and videos to learn pelvic diagnosis through the use of hand models. Hand models entail using both hands to represent the left and right innominates. With the third and fourth fingers curled in, the other fingers extend out to represent anatomical landmarks of the innominates (first finger is the posterior superior iliac spine, second finger is the anterior superior iliac spine, and fifth finger is the pubic ramus or medial malleolus) bilaterally. Using this model, students can move their hands to simulate the motion of the innominates. An online multiple choice question assessment was then administered at the end of the week. Four hundred and fifty one first year students were emailed an optional survey link containing four questions regarding the use of hand models in their education. The researchers evaluated the survey responses. The four survey questions were as follows:

1. Did you use hand models to help with your osteopathic pelvic diagnosis education?

2. How would you rate the helpfulness of hand models when learning pelvic diagnosis?

3. Did you use the hand models while taking the quiz today?

4. How would you rate the helpfulness of hand models while taking the quiz today?

Data Analysis: The data was collected using Research Electronic Data Capture (REDCap) and reported as descriptive statistics. Data was de-identified for participant privacy.
Results: Two hundred and nine out of 451 students responded to the survey, giving a $46.34 \%$ response rate.

Questions 1 and 2: 188 student respondents reported using hand models to assist with their osteopathic pelvic diagnosis education. Of those, 127 (67.55\%) student respondents rated the hand models "very helpful", 19 (10.10\%) student respondents rated the hand models "somewhat helpful”, $1(0.53 \%)$ student respondent rated the hand models "neutral", 6 (3.19\%) student respondents rated the hand models "somewhat unhelpful", 35 (18.61\%) student respondents rated the hand models "very unhelpful". Twenty one student respondents reported not using the hand models to learn pelvic diagnosis.

Questions 3 and 4: 169 student respondents reported using the hand models while taking the multiple choice assessment. Of those, 106 $(62.72 \%)$ student respondents rated the hand models "very helpful", 25 (14.79\%) student respondents rated the hand models "somewhat helpful", $1(0.59 \%)$ student respondent rated the hand models "neutral", 7 (4.14\%) student respondents rated the hand models "somewhat unhelpful”, $30(17.75 \%)$ student respondents rated the hand models "very unhelpful". Forty student respondents reported not using hand models while taking the multiple choice assessment.

Conclusion: Over $77 \%$ of student respondents reported the hand models were helpful for learning osteopathic diagnosis of the pelvis and helpful while taking the multiple choice assessment. These results highlight the utility of these models as effective simulations for innominate SD diagnosis. Student perspectives are imperative during this unprecedented time of online learning. As osteopathic medicine focuses on hands-on medicine, it is important for students to be able to effectively learn techniques when face-to-face 
opportunities are not available. Limitations include access to only NYITCOM first year students as well as the time sensitive nature of hand model implementation due to COVID-19 social distancing restrictions. Planned future research includes correlating student assessment scores for pelvic diagnosis questions with their satisfaction responses from this survey. Further research into the use of hand models for other anatomical regions - such as the sacrum and cranium - should be investigated. Qualifying and quantifying the utility of hand models may serve to standardize this OME teaching-learning tool for both in-person and remote learning.

Acknowledgment/Funding Source: This study, BHS-1543, was approved by the NYIT Institutional Review Board.

We would like to acknowledge Dr. Min-Kyung Jung for her assistance with statistical analysis.

\section{Reference}

1. DiGiovanna, E. L., Schiowitz, S., \& Dowling, D. J. (2005). An osteopathic approach to diagnosis and treatment. Philadelphia, PA: Lippincott Williams and Wilkins.

Poster No. *C61

Abstract No. 61

Category: Clinical

Research Focus Area: Chronic

Diseases \& Conditions

Understanding the Patient Population of a Student-Run Free Clinic: A Retrospective Chart Review

Sobiah Khan, OMS III ${ }^{1}$; Shayna Peterzell, OMS $\|^{1}$; Ashley Lovenduski, OMS II ${ }^{3}$; Shelly Sharma, OMS III ${ }^{1}$; Roma Padalkar, OMS III ${ }^{1}$; Isabella Slaby, DO²; Adarsh Gupta, DO ${ }^{3}$

${ }^{1}$ Rowan University School of Osteopathic Medicine, Stratford, NJ; ${ }^{2}$ Department of Internal Medicine, ChristianaCare Health System, Wilmington, DE; ${ }^{3}$ Department of Family Medicine, Rowan University School of Osteopathic Medicine, Stratford, NJ
Statement of Significance: R CHC (formerly $\mathrm{CCHC}$ ) provided patient care centered on osteopathic principles: considering all facets of a patient's life, such as socioeconomic status and resources available in the local community. Accessibility to affordable and quality treatments, such as giving referrals to the free OMM clinic at Rowan University, were considered in addition to other, less affordable treatments. This is a retrospective review of over $50 \%$ of the clinic medical records spanning 19 years of patient care, a microcosm of health and health care in Camden County. Although changes occurred both in the community, in the clinic, and in osteopathic medical practice over the course of all those years, the need for the clinic never dissipated.:

Research Methods: This is a retrospective review of the medical records of 1552 unique visits by 1182 individuals that received medical care at CCHC from 2000-2017. Institutional review board approval was obtained from the Rowan University School of Osteopathic Medicine Institutional Review Board prior to beginning the study. Permission to use the paper patient medical records was obtained from the RCHC. Medical records were selected randomly from the clinic medical record archive. Demographic (age, gender, race, town of residence) and medical (preexisting conditions, chief complaint, diagnosis, and management plan) information were collected and entered into a database using Qualtrics.

Data Analysis: Information from charts were extracted from Qualtrics into spreadsheets that were further transcribed and analyzed using Excel. All relevant categorical variables were described as percentages while means were calculated for continuous variables. Charts that did not have relevant data available (gender, duplicate entries or charts, etc.) were excluded from analysis. 
Results: The average age of the patient population was $33.42 \pm 17.47$ years (range: 1-80). 24.19\% individuals were under $18,44.16 \%$ between $18-40$, and $31.64 \%$ were over 40 years of age. $85.87 \%$ individuals visited the clinic once (1015/ $1182)$, while $14.33 \%$ (167/1182) made subsequent visits. 476 individuals were male, 671 were female, and 35 were categorized as other and/or undisclosed. Of those individuals that disclosed race or ethnicity, 351 identified as Hispanic and 199 as Black. Most clinic visitors were from Camden $(66 \%)$ while the remainder were from surrounding towns in the county and $6 \%$ of patients had prior primary care physicians as listed on their intake form.

Common pre-existing conditions $(501 / 1182)$ were asthma $(\mathrm{N}=125 / 501)$, hypertension $(\mathrm{N}=120 / 501)$, cardiovascular complications $(\mathrm{N}=69 / 501)$, and diabetes $(\mathrm{N}=42 / 501)$. The main services provided were physicals for work (29\%) and school (28\%) and associated laboratory work such as PPD skin testing. $32 \%$ of individuals came in for other complaints and $11 \%$ for well child visits. $30 \%$ of patients had a new diagnosis after their visit, most commonly dermatologic ( $\mathrm{N}=65 / 355)$, cardiac $(\mathrm{N}=65 / 355)$, respiratory $(\mathrm{N}=40 / 355)$, and reproductive conditions $(\mathrm{N}=32 / 355) .23 \%$ of patients were prescribed new medications and $42 \%$ of patients were prescribed lab tests.

Conclusion: $42 \%$ of clinic patients had a history of any pre-existing chronic condition. This is much higher than the Camden County rate of $16.3 \%$ [2]. However, rates for the most common conditions in the clinic population were comparable to county data. Overall, the clinic population health resembles that of the county.

$6 \%$ of patients had a prior primary care physician (PCP). This result indicates the need to further investigate if and why patients do not have access to a PCP. CCHC played an important role in providing primary care services. providing school and work physicals. A large percentage of patients came in for required work and school physicals. One could extrapolate that those who visited the clinic for required physicals likely do not have a PCP and/or do not visit them regularly as some individuals traveled over 100 miles and crossed state borders to be seen at the clinic. $86 \%$ of those patients that came for physicals did not make follow up appointments, further suggesting that these individuals likely do not visit a physician regularly or cannot afford to do so.

Due to inconsistent paper SOAP forms, there was inconsistent medical information collection. RCHC has implemented the use of EMR in response. This along with tailored intake forms (e.g., asking about access to food and shelter), $\mathrm{RCHC}$ will be able to provide individualized care and connect patients to appropriate services. There is a need for accessible and affordable primary care in the South Jersey area.

\section{Poster No. C64}

Abstract No. 64

Category: Clinical

\section{Research Focus Area: Chronic Diseases \& Conditions}

\section{Comparison of No Bacteria Reported on Microscopic Urinalysis and Culture Proven Group B Streptococcal Bacteriuria in a Pregnancy Population Maeve Gleason, DO ${ }^{1}$; Stephen M. Olson, DO²; Thomas L. Alderson, DO ${ }^{2}$ \\ ${ }^{1}$ McLaren Macomb, Mount Clemens, Ml; ${ }^{2}$ Department of Obstetrics and Gynecology, McLaren Macomb, Mount Clemens, MI}

Statement of Significance: The primary risk factor for neonatal early-onset GBS infection is maternal colonization. Administration of 
intrapartum antibiotic prophylaxis in high risk women for colonization has substantially reduced neonate infection. Two risk factors for maternal colonization are GBS bacteriuria (any colony count of GBS in urine) and GBS identified on a rectovaginal swab obtained between 36 and 37 weeks gestation (known as universal screening). Identifying high risk women allows osteopathic physicians to decrease neonatal early-onset GBS infection. GBS infection is the leading cause of early-onset neonatal sepsis and meningitis, with potential lifelong sequelae cerebral palsy, seizures, intellectual disability, hearing loss, and visual impairment.

Research Methods: The research entails retrospective chart review (institutional review board protocol number 2017-00049). Encounters (records/charts/patient care episodes) from female patients who presented to McLaren Macomb Family Birthing Center triage unit between October 1, 2016 and February 28, 2017 were reviewed. Collected data was part of the standard medical workup. As part of standard protocol, patient name, encounter number, and visit date were recorded on a hard copy log book in Triage. This log book was used to match patient encounter number to the hospital's Electronic Medical Record to obtain data. The data collected/recorded did not contain a link or code which could identify the patient directly or indirectly. Inclusion criteria included gestational age 20 0/7 weeks or greater at the time of presentation with documentation of a microscopic urinalysis and subsequent urine culture. Patients less than 18 years old were excluded from the study.

Data Analysis: Pearson's Chi-Square tests were conducted to examine the statistical associations between the frequencies of bacteria in the microscopic urinalysis and the frequencies of bacteria using alternative methods. These tests determined if the observed frequencies deviated from the frequencies expected by random chance, assuming that the expected frequencies in at least $80 \%$ of the cells of the cross-tabulations were greater than 5. In this study, Cramer's V was used as the effect size, to measure the strength of the association between the frequencies of the bacteria in the microscopic urinalysis and the frequencies of the presence of bacteria using alternative methods. Cramer's V ranges from 0 to 1 where $<0.10$ represents no correlation; 0.1 to 0.3 is a weak correlation; 0.3 to 0.5 is a moderate correlation; and $>0.5$ is a strong correlation.

Results: A cross-tabulation of the frequencies of the bacteria in the microscopic urinalysis vs the frequencies of the presence of bacteria in urine cultures was analyzed. Pearson's Chi Square test $\left(\chi^{2}(6)=13.59, p=0.034\right)$ indicated that the presence of bacteria in the urine culture was probably dependent on (i.e., associated with) the results of the microscopic urinalysis. However, Cramer's V $=0.225$ reflected only a weak correlation. A second cross-tabulation of the frequencies of the bacteria in the microscopic urinalysis vs the frequencies of the presence of low colony count GBS bacteria in urine cultures was analyzed. Pearson's Chi Square test $(\chi 2(5)=5.80, \mathrm{p}=$ 0.326 ) indicated that the presence of GBS bacteria in the urine culture was probably independent of (i.e., not associated with) the results of the microscopic urinalysis. Cramer's V $=0.197$ reflected only a weak. Finally, a cross-tabulation of the frequencies of the bacteria in the microscopic urinalysis vs the frequencies of the presence of low colony count GBS bacteria in rectovaginal swabs was analyzed. Pearson's Chi Square test $(\chi 2(6)=$ $7.96, p=0.241)$ indicated that the presence of bacteria in the rectovaginal swabs was probably independent of (i.e., not associated with) the results of the microscopic urinalysis. Cramer's $\mathrm{V}=0.224$ reflected only a weak. 
Conclusion: The research hypothesis was supported because the results of the microscopic urinalysis were not strongly correlated with the results of low colony-count GBS growth on the urine cultures or rectovaginal swabs. Therefore, a negative microscopic urinalysis may not positively identify women at high risk for GBS colonization.

Acknowledgment/Funding Source: Special thank you to Dr. Ron Fisher for providing the statistical analysis.

\section{References}

1. Prevention of Perinatal Group B Streptococcal Disease. Centers for Disease Control and Prevention. MMWR 2010;59.No.RR-10.

2. Prevention of group $B$ streptococcal early-onset disease in newborns. ACOG Committee Opinion No. 797. American College of Obstetricians and Gynecologists. Obstet Gynecol 2020;135:e51-72.

3. Edwards, MS, Rench, MA, Haffar, AA, Murphy, MA, Desmond, MM, Baker, CJ. Long-term sequelae of group B streptococcal meningitis in infants. J Pediatr. 1985;106(5):717-722. doi:10.1016/s0022-3476(85)80342-5

\section{$\star$ Poster No. *C65}

Abstract No. 65

Category: Health Services

Research Focus Area: Osteopathic Philosophy

Implementation of a Novel Dual Video Telehealth Platform During a Cardiology Clerkship During COVID-19 Pandemic:

\section{TeleMedstudent}

Zachary Coopee, OMS III; Nicole K. Hunzeker, OMS II; O. Robert Hubley, OMS II; Slava Gitelman, OMS IV; Todd J. Cohen, MD

New York Institute of Technology (NYIT), Old Westbury, NY

Statement of Significance: During the unfortunate global setting of COVID-19, third and fourth year medical students have been relegated to learning from at-home modules, question banks, and patient case reviews. These methods have diverged from the core osteopathic principles of patient-based medicine that are the backbone to becoming an osteopathic physician. Development of a dual-platform telehealth system allows osteopathic medical students to continue safely interacting with patients during the present pandemic and further their patient-based osteopathic approach ensuring that our future osteopathic physicians are competent and prepared for future pandemics.

Research Methods: All patients gave verbal consent for medical student participation, and only HIPAA compliant video platforms were utilized. Protocol BHS-1465, "Diagnostic and Therapeutic Outcomes from the Long Island Heart Rhythm Center" was approved by the NYIT IRB which permitted the clinic to look back retrospectively on the use of telehealth in the clinic. The use of the system was with respect to medical student participation, and continued engagement were assessed. Data was then collected from patients participating in the TeleMedstudentTM system at the Long Island Heart Rhythm Center and analyzed for quality of engagement by the osteopathic medical student.

Data Analysis: Descriptive statistics were performed when appropriate. Each patient's age, sex, diagnosis, and means of communication were all documented. All patients were averaged for mean age and sex.

The osteopathic medical student evaluated each patient encounter on a scale of one to five. One being no patient interaction. Two being minimal communication with the patient. Three being communication with the patient about the encounter and troubleshooting technical difficulties. Four indicates the student spoke to the patient, confirmed their chief complaint and asked their 
Table 1.

Quantification of Osteopathic Medical

Student Engagement

\begin{tabular}{ll}
$\begin{array}{l}\text { Osteopathic Student } \\
\text { Engagement Rating (1-5) }\end{array}$ & $\begin{array}{l}\text { Number of } \\
\text { Encounters }\end{array}$ \\
\hline Level 1 & 0 \\
\hline Level 2 & 0 \\
\hline Level 3 & 4 \\
\hline Level 4 & 47 \\
\hline Level 5 & 60 \\
\hline Total Patient Encounters & 111 \\
\hline Average Student Engagement & 4.51
\end{tabular}

history and medications. Five represents the student fully gathered all relevant patient information and actively recorded it into the electronic medical record.

The score for all the medical student's patient encounters were then averaged.

Results: From March 2020 to July 2020 during the COVID-19 pandemic in the epicenter (New York city and state), the Long Island Heart Rhythm Center saw 111 patients remotely, together with the student on clerkship. The medical student on clerkship (SG) participated in $100 \%$ of scheduled clinics, and as many emergent add on patients as possible during that period of time. These patients consisted of 31 males, 80 females, with a mean age of 52.1 years ranging from 18 to 88 years old (Table 1). Out of the 111 patient encounters $79 \%$ were conducted via video conferencing and $21 \%$ were via phone conferencing. The most prevalent cardiac symptoms were palpitations, presyncope, and syncope. Many of the patients had implantable devices including pacemakers, implantable loop recorders, and implantable defibrillators. All patients agreed to medical student remote participation (except one). The osteopathic medical student evaluated each encounter on their level of patient engagement on a scale of one to five, with one being minimal patient engagement and five being the highest level of patient engagement, respectively. Out of the 111 encounters, the student had an average patient engagement level of 4.51 (Table q).

Conclusion: A novel dual video telehealth platform such as TeleMedstudentTM can be successfully utilized in order to continue clerkships with real patients remotely during a pandemic at an osteopathic medical school. This novel telehealth methodology that we developed emphasizes the osteopathic principles of patient based medicine while respecting the health and safety of the patient and students respectively.

Poster No. *C66

Abstract No. 66

Category: Clinical

\section{Research Focus Area: Chronic Diseases \& Conditions}

\section{Lack of Vaccination Coverage in the Patient Population of a Student-Run}

\section{Free Clinic}

Sobiah Khan, OMS III ${ }^{1}$; Shayna Peterzell, OMS II'; Ashley Lovenduski, OMS II ${ }^{1}$; Shelly Sharma, OMS III'; Adarsh Gupta, DO ${ }^{2}$

${ }^{1}$ Rowan University School of Osteopathic Medicine, Stratford, NJ; ${ }^{2}$ Department of Family Medicine, Rowan University School of Osteopathic Medicine, Stratford, NJ

Statement of Significance: Following the recommended vaccination schedules for children and adults in the United States requires that an individual be able to afford periodic visits to a physician as well as the vaccines themselves. It also requires that an individual be aware of the importance of 
vaccinations. This study shows the low vaccination rates of individuals that utilized a student-run free clinic over the course of 19 years. Thus, this study highlights a gap in care, education, and/or accessibility among a unique segment of the population. Osteopathic medicine requires use of all tools available in our armamentarium when providing medical care (whether it be OMT or vaccines), and this data suggests this is not occurring.

Research Methods: This is an IRB-approved retrospective chart review of individuals that visited CCHC for medical care. Demographic information (age, gender, town of residence) and vaccination records were abstracted from charts with the permission of RCHC. Vaccination records were reviewed for a history of at least one vaccination of DTap, IPV, HiB, HepB, MMR, and Varicella. All other vaccinations (MenB, PCV13/ PPSV23, HPV, and the annual flu vaccine) were initially classified as "other." County data were obtained from New Jersey State Health Assessment Data (NJSHAD) and the New Jersey Department of Health Local Health Department and School Audit Data [2]. National data were obtained from the Centers for Disease Control and Prevention National Health Interview Survey (NHIS) $[1,3,4]$.

Data Analysis: Data were extracted from Qualtrics and further analyzed in Excel. Means were calculated for continuous variables, while all categorical variables were described as percentages and frequencies. In further analysis, frequencies of appropriate categories were further compared with Centers for Disease Control and New Jersey Department of Health vaccination data. Charts that did not include vaccination data were excluded from final analysis.

Results: 1182 individuals received medical care at the clinic. $24.20 \%$ children $(<18)$ and $75.80 \%$ adults $(>18)$ visited the clinic. The average age of children that were provided medical care at the clinic was $13 \pm 4.15$ years while that of adults was $40 \pm 14.91$ years. 476 individuals were male, 671 were female, and 35 were categorized as other and/or undisclosed. $68 \%$ of services provided were physicals or well-child visits. $85.87 \%$ individuals visited the clinic once $(1015 / 1182)$, while $14.33 \%$ (167/1182) made follow up visits. $87.22 \%$ were from Camden County and $6 \%$ of patients had prior primary care physicians as listed on their intake form.

$39 \%(460 / 1882)$ charts included vaccination records and/or vaccination history. Of those charts that included any immunization history, $30.87 \%$ of children $(142 / 460)$ and $69.13 \%$ of adults $(318 / 460)$ had a history of at least one vaccination. Vaccination coverage for the whole population was as follows: $70.65 \%$ DTap, 65.65\% IPV, 45.65\% HiB, 67.61\% HepB, 70.87\% MMR, 63.26\% Varicella, and 23.04\% other. Of the $\mathrm{N}=186$ "other" vaccines, $30 / 186$ were MenB (meningococcal), 23/186 HPV, 31/ 186 were pneumococcal pneumonia, and 41/186 were HepA (hepatitis A). Only 30/460 individuals with vaccination records received the flu vaccine at least once. $49.65 \%$ of all children $(286 / 1182)$ that received medical care at the clinic were noted to be up-to-date on their vaccinations.

Conclusion: Vaccination coverage was lower among the clinic population than the county and national rates across all combined 7-series 
vaccines $[1,2,3]$. Immunization rates for each individual recommended vaccine is lower among the clinic population suggesting that there is an accessibility, affordability, or education issue. Additionally, influenza vaccinations were very low $(6.5 \%)$ despite being recommended annually. HPV vaccine rate was only $5 \%$ while national rates hover around $39.9 \%$ [4].

Of 286 children, only $49.65 \%$ were noted to have up-to-date vaccinations, much lower than local public school rates. The remainder of the charts were either missing this information or made no mention of immunization status. This suggests a lapse in care by the volunteer medical staff at the clinic as immunizations are an essential part of pediatric health care.

With the reopening of $\mathrm{RCHC}$, the results of the vaccination review guides how to move forward in order to mediate these issues. The clinic has partnered with local organizations to ensure proper referrals that are free/affordable in regards to immunization services. RCHC is also in the process of providing certain immunizations on site for patients as well as developing educational programs in order to increase awareness. As the data shows a clear need for increasing immunizations and establishing primary care relationships, RCHC ultimately strives to continually advocate, educate, and provide care for the underserved in Camden County.

\section{References}

1. VaxView. (2016, October 06). Retrieved July 10, 2020, from https://www.cdc.gov/vaccines/vaxview/index.htm

2. $\mathrm{DOH}$ (n.d.). New Jersey State Health Assessment Data New Jersey's Public Health Data Resource. Retrieved July 10, 2020, from https://www-doh.state.nj.us/doh-shad/home/Welcome.html

3. FastStats - Immunization. (2017, March 17). Retrieved July 10, 2020, from https://www.cdc.gov/nchs/fastats/immunize.htm

4. Boersma, P, Black, LI. Human papillomavirus vaccination among adults aged 18-26, 2013-2018. NCHS Data Brief, no 354. Hyattsville, MD: National Center for Health Statistics. 2020.
Poster No. *C67

Abstract No. 67

Category: Clinical

Research Focus Area: Pain

Management

The Effect of Osteopathic

Manipulative Treatment on Stiff

Person Syndrome

Michael Ogene Bazzi, OMS OMS IV ${ }^{1}$; Adithi Vemuri, OMS III ${ }^{1}$; Michael A. Downing, OMS III ${ }^{1}$; Patrick

E. Barry, $\mathrm{DO}^{2}$

${ }^{1}$ Nova Southeastern University Dr. Kiran C. Patel College of Osteopathic Medicine, Fort Lauderdale, FL ${ }^{2}$ Osteopathic Manipulative Medicine Clinic, Nova Southeastern University Dr. Kiran C. Patel College of Osteopathic Medicine, Fort Lauderdale, FL

Statement of Significance: This case utilized a holistic approach through use of osteopathic manipulative treatment (OMT), as an alternative or adjunct to medication, to improve the quality of life in a patient experiencing chronic pain secondary to a rare neurological condition.

Research Methods: A 61-year-old Caucasian female previously diagnosed with SPS presented to Nova Southeastern University's Osteopathic Manipulative Medicine (OMM) clinic with complaints of diffuse pain, most severe in the low back. The pain had an insidious onset, described as constant stiffness, but waxing and waning in severity. On her initial visit she reported 3/10 pain at rest and $8 / 10$ pain during exacerbation which occurred with certain movements and activities. The patient reported that she experiences intermittent paresthesia in the lower extremities as well as difficulty with voiding, sometimes taking 10 minutes before being able to urinate. On physical examination the patient was alert and oriented $\mathrm{x} 3$ but presented with significant lid lag and dilated pupils. Postural examination showed kyphosis of the thoracic spine and a scoliotic curve in the lumbar spine. 
Musculoskeletal and Neurological examinations revealed upper and lower extremities had 4/5 muscle strength, 2/4 deep tendon reflexes, and dermatomes intact to light touch at L2-S1 and C5-T1 bilaterally. The patient was seen for a total of four visits, each appointment three weeks apart. During each appointment, somatic dysfunctions were discovered in the following areas: Occipitoatlantal (OA) joint, cervical vertebrae, thoracic vertebrae, lumbar vertebrae, ribs, innominates, and the shoulder girdle. These somatic dysfunctions were treated with a variety of osteopathic techniques including muscle energy, direct MFR, and BLT.

Data Analysis: Subjective information was utilized to determine somatic dysfunctions as well as patient-reported pain, functionality, and relief. All diagnosed somatic dysfunctions are recorded prior to treatment, then reassessed after treatment as either resolved, improved, unchanged, or worsened.

Results: Following each visit, the patient reported a consistent improvement in overall pain levels: 2/ 10 resting pain (3/10 prior) and 4/10 pain during exacerbation ( $8 / 10$ prior). In addition, she stated that she experienced "almost normal" urination that lasted several days following each treatment session. After each of the treatment sessions, the patient reported transient soreness and fatigue. Following the patient's fourth treatment, she claimed that other treatment modalities she had attempted prior were unsuccessful and the only treatment that was currently providing her relief was OMT.

Conclusion: This case demonstrates that the use of OMT on patients with Stiff Person Syndrome (SPS) may be effective in alleviating pain and normalizing autonomic responses through the treatment of somatic dysfunctions with techniques such as muscle energy, direct MFR, and BLT.

\section{Poster No. ${ }^{*} \mathrm{C} 69$}

Abstract No. 69

Category: Clinical

Research Focus Area: Osteopathic Philosophy

\section{Undergraduate Pre-Medical Students}

Attitudes Regarding Osteopathic Medicine and Awareness Level of New Osteopathic Medical Schools Alexander Paiva, OMS III, MA, LMHC ${ }^{1}$; Carol S. Browne, $\mathrm{DO}^{2}$

${ }^{1}$ University of the Incarnate Word School of Osteopathic Medicine, San Antonio, TX; ${ }^{2}$ Department of Clinical and Applied Science Education,, University of the Incarnate Word School of Osteopathic Medicine, San Antonio, TX

Statement of Significance: The purpose of this study is to examine the attitudes of undergraduate pre-medical students, towards osteopathic medicine. In addition, the study will examine the level of awareness of undergraduate students regarding the existence of a new local osteopathic medical school. The study goal is to determine if the growth of osteopathic medicine is improving the level of awareness of the field within the communities in which new schools are created.

Research Methods: Students were contacted at four local academic institutions with permission from the non-UIW institutional review boards. Recruitment emails with an attached online survey in a Microsoft Forms format were sent to pre-medical student groups, pre-medical advising offices, identified pre-medical students, and department chairs associated with pre-medical prerequisites, such as biology and chemistry. The survey included demographic information questions and three sets of five Likert Scale questions 
for a total of 15 questions. The question categories were: (1) students' attitudes regarding osteopathic medicine; (2) experiences with seeing an osteopathic physician; and (3) levels of awareness of new local and regional osteopathic schools, Participants were able to view the consent form and survey online and were given an electronic gift card for participating in the survey.

Data Analysis: Demographics were collected, including gender, grade level, and associated school. All non-UIW students were grouped together. Chi-Square analysis was conducted to determine differences between UIW and non-UIW students. Total responses to each question were compiled and graphed, in addition to splitting total responses into those from UIW vs Non-UIW students. Comparison statistics were performed using one tailed $\mathrm{T}$ test analyses ( $p$-value $=0.05)$ assuming unequal variances to examine the responses of UIW to Non-UIW students for each separate question. One-tailed $\mathrm{T}$ tests were also performed on collective totals based on the question categories. Analysis was completed using Microsoft Excel Data Analysis Tool and Statistical Program for Social Sciences (SPSS) software.

Results: The following are the survey results: Demographics: 51 total respondents. Duplicate entries and unverified students via their school email address were excluded, leaving a total of 37 participants. Gender: 7 males, 30 females; School Distribution: 25 from UIW, 12 from non-UIW schools; Class year distribution: 16 Freshman, 8 Sophomores, 8 Juniors, 5 seniors. Chi-square UIW vs Non-UIW (2): $\mathrm{p}=0.51$;

$\mathrm{T}$ tests broken down by each question and category, comparing UIW to Non-UIW students: (question number and degrees of freedom (subscript), T-score, P-value):
T test for Q1 (df=4): 0.872, $\mathrm{p}=0.216$; $\mathrm{T}$ test for Q2 $(\mathrm{df}=) 4$ : $0.956, \mathrm{p}=0.197$; $\mathrm{T}$ test for $\mathrm{Q} 3(\mathrm{df}=) 4$ : $1.033, \mathrm{p}=0.180$;

T test for Q4 $(\mathrm{df}=) 6: 1.169, \mathrm{p}=0.143$; T test for Q5 $(\mathrm{df}=5): 1.343, \mathrm{p}=0.119$;

$\mathrm{T}$ test for Total Attitude Category $(\mathrm{df}=6): 1.131$ $\mathrm{p}=0.151$;

T test for Q6 $(\mathrm{df}=7)$ : 1.148, $\mathrm{p}=0.144$; T test for Q7 $(\mathrm{df}=7)$ : 1.046, $\mathrm{p}=0.165$; $\mathrm{T}$ test for $\mathrm{Q} 8(\mathrm{df}=7)$ : $1.030, \mathrm{p}=0.169$;

T test for Q9 $(\mathrm{df}=8)$ : 1.245, $\mathrm{p}=0.124$; $\mathrm{T}$ test for Q10 (df=6): 0.855, p=0.213;

$\mathrm{T}$ test for Total Experiences Category $(\mathrm{df}=7)$ : $1.243, \mathrm{p}=0.127$;

T test for Q11 $(\mathrm{df}=5)$ : 1.039, $\mathrm{p}=0.173$; $\mathrm{T}$ test for Q12 $(\mathrm{df}=5)$ : $0.701, \mathrm{p}=0.257$; $\mathrm{T}$ test for $\mathrm{Q} 13$ $(\mathrm{df}=5): 0.993, \mathrm{p}=0.183$;

T test for Q14 $(\mathrm{df}=5)$ : $1.906, \mathrm{p}=0.057$; $\mathrm{T}$ test for Q15 (df=5): 1.083, p=0.164;

$\mathrm{T}$ test for Total Awareness Category $(\mathrm{df}=5)$ : $1.081, \mathrm{p}=0.164$

Conclusion: Raw responses showed a more positive trend from UIW students with higher awareness of the new osteopathic school and having more positive attitudes towards osteopathic medicine, which was predicted given the university affiliation. The study did not show significant differences in responses comparing UIW and Non-UIW students regarding their attitudes towards osteopathic medicine, experiences with osteopathic medicine, and awareness levels of new osteopathic medicine schools. The study was limited due to the small number of respondents, as well as the impersonal method of an online survey. Other limitations included lack of affiliation with other undergraduate institutions, which may have 
helped with recruitment efforts. Improvement with recruitment efforts could benefit the significance of study data, including have more distribution from pre-medical advising offices. There may also have been some association bias, considering that osteopathic students have presented panel discussions at a couple local undergraduate institutions. In addition, further studies with larger sample sizes inclusive of other cities and universities with new affiliated osteopathic medical schools, are warranted to determine if the understanding of osteopathic medicine is prevalent among undergraduate students and the general public, particularly as new schools open, which could benefit the growth of the field of osteopathic medicine.

Acknowledgment/Funding Source: Steven Mifflin, $\mathrm{PhD}$, Associate Dean for Research and Innovation, University of the Incarnate Word School of Osteopathic Medicine (UIWSOM) for providing a grant fund for the study from the UIWSOM Research Fund.

IRB Approval: Approved on August 26, 2019 by the University of the Incarnate Word Institutional Review Board, FWA00009201; Approval number 19-18-001.

\section{References}

1. American Association of Colleges of Osteopathic Medicine, Research Department. 2018. Trends in Osteopathic Medical School Applicants, Enrollment, and Graduates. Available at (https://www.aacom.org/reports-programs-initiatives/ aacom-reports/student-enrollment) Accessed June 30, 2019.

2. Stamat, HM, et al. Osteopathic Medicine and Community Health Fairs: Increasing Public Awareness While Improving Public Health. J Am Osteopath Assoc. 2008;108:397-403.

3. American Association of Colleges of Osteopathic Medicine Available at: (https://www.aacom.org/become-a-doctor/us-coms). Accessed July 5, 2019.

4. Shannon, ST and Teitelbaum, HM. The Status and Future of Osteopathic Medical Education in the United States. Acad Med. 2009; 84:707-711.

5. Licciardone JC. Awareness and Use of Osteopathic Physicians in the United States: Results of the Second Osteopathic Survey of Health Care in America (OSTEOSURV-II) J Am Osteopath Assoc. 2003; 103:281-289.
Poster No. *C73

Abstract No. 73

Category: Health Services

Research Focus Area: Chronic

Diseases \& Conditions

Training Osteopathic Medical

Students for Drastic 2021 Health

Record Documentation Policy

\section{Changes}

Ramandeep Bains, OMS I; Avleen Gill, OMS I, MS; Jagmeet Singh, MS; Andrew Antos, MS; Sean Lim, OMS III; Heidi Molga, OMS; Ayesha Malik, OMS II; Susan St. Pierre, DO; Glenn Davis, MS;

Michael Warner, DO, CPC, CPCO, CPMA, AAPC Fellow

Touro University College of Osteopathic Medicine

(California), Vallejo, CA

Statement of Significance: Osteopathic medicine has traditionally viewed the body as a unit. Understanding the patient includes gathering the patient's needs, concerns and experiences in order to make an accurate diagnosis and formulate an effective treatment plan. Rational treatment, for acute problems and chronic disease management, is dependent on effective patient-provider communication. In light of emerging policies, which eliminate requirements to document the History or Examination in outpatient health records, we need to be certain the patient's History and Exam are not clinically disregarded.

Research Methods: Osteopathic medical students, while displaced from clinical rotations due to the COVID-19 pandemic, were offered a $3 \frac{1}{2}$ hour mini-course as part an accommodative curriculum to understand existing and emerging federal health record documentation policy changes. The course viewed issues through the lens of American Osteopathic Association (AOA) Core Competencies: Systems-Based Practice (SBP) and Practice-Based Learning \& 
Improvement (PBLI). Surveys, administered through a QualtricsTM generated email after each of 4 sessions, were approved by Touro University California's Institutional Review Board [M-0920].

Participation in the Zoom based mini-course began with a brief Introduction, followed by a survey and an assignment to view a 32-minute AOA Evaluation and Management scoring webinar. A 45-minute presentation on the next day explored issues from a SBP viewpoint: explaining 'why' current policies are in place and 'why' new changes are occurring. After the SBP session and survey, participants were invited to complete a History on themselves utilizing a free online patient empowerment tool at www.PreHx.com. The following day, another 45-minute session addressed 'how' to accommodate policy changes through the lens of PBLI. After this session and survey, participants were assigned to review a 2017 JAOA article where patients were invited to author the History component of their own health record. The final session reviewed survey results and conducted active discussion.

Data Analysis: Surveys sent to osteopathic medical students were analyzed by the University's QualtricsTM software. As per IRB protocols, all data was de-identified prior to analysis.

Results: 79 of a class of 134 students consented to participate in the first survey, which revealed 58\% learned on the day of education that starting January 1, 2021 documentation of the History and Examination in outpatient health records will no longer be linked to provider payment. $43 \%$ strongly agreed and $36 \%$ agreed in the belief that documentation of the History is important to determining an accurate diagnosis, formulating an effective treatment plan and sharing information with all health care professionals. After the minicourse, $80 \%$ agreed/strongly agreed to feeling optimistic that health record documentation policies are going to make the practice of medicine more meaningful for patients and providers. $68 \%$, with agreed and strongly agreed responses, support this type of training for all medical students. In addition, $85 \%$ of study participants believe guidelines need to be offered in response to these changing policies so providers, ancillary medical staff members and patients can best function.

Conclusion: This study identifies a need to educate medical students of drastic 2021 health record documentation policy changes. While osteopathic medical students feel confident new policies will improve the practice of medicine for all stakeholders, they recognize a need for training and guidelines.

Acknowledgment/Funding Source: We thank Touro University California for taking a leadership role in health record documentation policies and practice.

\section{References}

1. Warner, MJ, Simunich, T, Warner, MK, Dado, J “Use of Patient-Authored Prehistory to Improve Patient Experiences and Accommodate Federal Law," JAOA, February 2017 https://jaoa. org/article. aspx? articleid $=2599978$

2. Warner, M, Penkala, C "Evaluation and Management (E/M) Documentation and Scoring," 32-min AOA webinar posted on YouTube October 12, 2018 https://www.youtube.com/watch? $v=-C j J 4 M Z t B e c \& f e a t u r e=y o u t u . b e$

3. Warner, M "Death of the Documented History, Rise of the Patient Advocate," Healthcare Business Monthly magazine, AAPC, Nov 2019 (digital version at AAPC's Knowledge Center) https://www. aapc.com/blog/49124-death-of-the-documented-history-rise-ofthe-patient-advocate/

4. Baker, L "2021 Changes for Evaluation and Management Services" The National Law Review, February 26, 2020 (posted June 16, 2020) https://www.natlawreview.com/article/ 2021-changes-evaluation-and-management-services\#: : text=Starting $\% 20$ January $\% 201 \% 2 \mathrm{C} \% 202021 \% 2 \mathrm{C} \% 20$ the, history\%20and\%2For\%20examination.\%E2\%80\%9D 
Poster No. C74

Abstract No. 74

Category: Basic Science

Research Focus Area: Impact of

Osteopathic Manipulative Medicine

(OMM) \& Osteopathic Manipulative

Treatment (OMT)

AOA Grant Number: 1915733

Cranial Manipulation Affects

Neuronal Gene Expression,

Explaining the Improved Cognitive

Ability in an Animal Model of Age-related Cognitive Decline

Ramu Anandakrishnan, PhD

Edward Via College of Osteopathic Medicine

(Virginia), Blacksburg, VA

Statement of Significance: Neurodegeneration with age is a devastating disorder afflicting a rapidly growing older population around the world. Although there is no cure for it or even a treatment to slow its progression, pharmacological and non-pharmacological therapies, such as COM, can treat the associated symptoms. However, $\mathrm{COM}$ is not generally prescribed for treating neurodegenerative disorders due to limited evidence for its efficacy, and the lack of a sound scientific explanation. Here we show that COM may be an effective treatment for these disorders, and provide a molecular level mechanistic explanation for the observed cognitive improvement.

Research Methods: We use an animal model for age-related cognitive decline (aged rats) to determine if COM can improve cognitive ability. The cognitive ability of 18 animals ( 9 treated and 9 untreated) were measured using the Morris water maze assay. The assay consisted of four days of training followed by four days of testing. Following the tests, the animals were euthanized and tissue samples collected from the dorsal end prefrontal cortex. RNA was extracted from the tissue samples and sequenced to determine the effect of COM treatment on gene expression levels.

Data Analysis: Videos of the water maze assay were analyzed using software from ANImaze, to calculate time, distance, speed and paths taken by individual animals. Statistical significance of differences in performance between treated and untreated animals was determined by 2-tailed unpaired $t$ test. Gene expression levels were calculated from RNA sequencing reads using the Cufflinks software suite. Statistical significance of differences in gene expression between samples from treated and untreated animals was determined by the false discovery rate (Benjamini-Hochberg procedure).

Results: We show that, in an animal model for age-related cognitive decline (aged rats), COM significantly affects the expression of 54 genes in the neuronal pathway $(\mathrm{FDR}<0.004)$, which can explain their better performance on the Morris water maze assay compared with untreated animals (p-value $<0.01$ ). In addition, $40 \%$ of the most significant differentially expressed genes have been previously implicated in neurological disorders.

Conclusion: There is extensive evidence that mechanical stress can stimulate activity in neuronal cells. We speculate that COM induced change in intracranial pressure promotes the differential expression of neuronal pathway genes and improved cognitive ability. The differentially expressed genes provide a mechanistic explanation for the reported benefits of $\mathrm{COM}$ and justification for a robust double-blind placebo-controlled clinical trial to investigate $\mathrm{COM}$ as a therapeutic option for neurodegenerative disorders. With clinical validation, COM could represent a much needed therapy for these debilitating disorders.

Acknowledgment/Funding Source: AOA grant \#1915733

VCOM REAP grant BC 2019 
Poster No. *C75

Abstract No. 75

Category: Clinical

Research Focus Area: Chronic

Diseases \& Conditions

\section{Evaluation of the Diabetic}

Ketoacidosis Management in the

Critically IIl Children and Adolescents

Mackenzie Dreher, OMS IV, MPH ${ }^{1}$; Brittany Skaggs,

OMS IV ${ }^{1}$; Christine Angeles, OMS IV ${ }^{1}$;

Alyssa DaVolio, OMS IV ${ }^{1}$; Jacob Andrews, OMS IV ${ }^{1}$; Ning Cheng, MS, $\mathrm{PhD}^{1}$; Hanna Sahhar, MD, FAAP, FACOP $^{2}$

${ }^{1}$ Edward Via College of Osteopathic Medicine (South Carolina), Spartanburg, SC; ${ }^{2}$ Department of

Pediatrics, Edward Via College of Osteopathic Medicine (South Carolina--Carolinas Campus and Spartanburg Regional Healthcare System (SRHS), Spartanburg, SC

Statement of Significance: DKA diagnostic biochemical criteria includes blood glucose level $>200 \mathrm{mg} / \mathrm{dL}$, metabolic acidosis with $\mathrm{pH}<7.30$ or serum bicarbonate level $<15 \mathrm{mEq} / \mathrm{L}$, and ketonemia/ketonuria. Pediatric T1DM patients have a $1-10 \%$ risk per year of developing DKA. Without resolving symptoms quickly and avoiding complications, morbidity for this population increases. A tenet of osteopathic medicine states, "rational treatment is based upon an understanding of the basic principles of body unity, self-regulation, and the interrelationship of structure and function." DKA is a manifestation of multiple regulator systems and hormones within a patient, which are accounted for within every step of treatment; without these guidelines, severe complications may result.

Research Methods: A retrospective medical record review of 247 patients, ages 0 to 17 years old, admitted to the Pediatric Intensive Care Unit (PICU) of Spartanburg Regional Health System met the diagnostic criteria for diabetic ketoacidosis (DKA).
Data Analysis: Descriptive statistical analysis, simple linear regression, and Fisher's exact test were performed to assess for significance $(\mathrm{p} \leq 0.05)$ using SPSS Data Analysis Software.

Results: The study included 247 patients, 55.9\% female and $44.1 \%$ male, $48.8 \%$ white and $48.4 \%$ African American. T1DM encompassed 97.6\% and $28.3 \%$ of all patients had new onset diabetes. Population age groups were 13-17 years old at $60.7 \%$ followed by $6-12$ years old at $32.8 \%$.

Primary outcomes analyzed the average time to correct metabolic acidosis (serum bicarbonate $>14.99 \mathrm{mEq} / \mathrm{L}), \quad 5.98 \pm 9.58$ hours, and the average amount of fluids and insulin per kilogram to correct dehydration (serum bicarbonate $>17.99$ $\mathrm{mEq} / \mathrm{L})$ at $114.38 \pm 148.72 \mathrm{~mL} / \mathrm{kg}$ and $2.6 \pm 5.5$ units $/ \mathrm{kg}$, with a mean drip rate of $0.098 \pm 0.174$ units $/ \mathrm{kg} / \mathrm{hr}$.

Secondary outcomes analyzed average length of stay (LOS), complications of admission (arrhythmias, cerebral edema, and hypoglycemia), and change in heart rate (HR) from initial presentation to correction of acidosis and resolution of dehydration. The PICU LOS averaged 1.54 \pm 1.06 days. Complication incidents were cerebral edema $4 \%$, arrhythmias $2 \%$ and hypoglycemia $1.6 \%$. Further analysis related complications to a factor during initial emergency room management: administration of insulin bolus, sodium bicarbonate, $\geq 2$ fluid boluses at $20 \mathrm{ml} / \mathrm{kg}$ each, or a fluid bolus $>20$ $\mathrm{ml} / \mathrm{kg}$. The incidence of cerebral edema was significantly related to the use of sodium bicarbonate $(p=0.034)$. Lastly, significance was noted between the decrease in HR from baseline to correction of acidosis and baseline HR to resolution of dehydration; with an average decrease of 35 and 50 beats per minute respectively $(\mathrm{p}<0.001)$. 
Conclusion: Following DKA protocol in the PICU is imperative to minimize DKA complications such as cerebral edema, while also reducing the amount of time a patient spends admitted to the PICU. Results showed complications of cerebral edema were related to administration of a sodium bicarbonate bolus in the emergency department. The duration of time to resolve metabolic acidosis and the amounts of fluids and insulin to correct for dehydration are reported in this study with emphasis on continuing the fluid and insulin treatment until the resolution of the dehydration, rather than just the correction of metabolic acidosis.

Acknowledgment/Funding Source: This study was approved by Spartanburg Regional Healthcare System Institutional Review Board

Poster No. *C76

Abstract No. 76

Category: Clinical

\section{Research Focus Area: Chronic} Diseases \& Conditions

Evolving from the ABCDEs of Early Melanoma Detection: A Case Series Utilizing Pigmented Lesion Assays and Non-Invasive Imaging to Minimize

\section{Biopsies}

Moshe Y. Bressler, OMS IV ${ }^{1}$; Melissa Gill, MD²; Orit Markowitz, $\mathrm{MD}^{3}$

${ }^{1}$ New York Institute of Technology (NYIT), Old Westbury, NY; ${ }^{2}$ Department of Pathology, SUNY Downstate, New York Institute of Technology (NYIT), Old Westbury, NY; ${ }^{3}$ Department of Dermatology, Icahn School of Medicine at Mount Sinai, New York, NY

Statement of Significance: Melanoma is a leading cause of cancer deaths in young adults, age is leading risk factor. Early detection and reducing unnecessary biopsies aligns with the osteopathic value of preserving cutaneous structure and function.

The PLA uses adhesive tape to detect gene expression and rule out melanoma and detects two genes, PRAME (preferentially expressed antigen in melanoma) and LINC00518 ${ }^{2}$. PRAME is highly associated with melanoma and is not produced by healthy cells except for select glandular tissues ${ }^{3}$. LINC00518 is associated with cell growth, invasion, and metastasis of melanoma, breast, and cervical cancer. Increased levels in melanoma patients correlate with poorer survival ${ }^{4}$, however its presence in benign nevi is poorly understood.

Research Methods: Patients with pigmented lesions suspicious for melanoma were offered a DermTech PLA tape-strip assay or an alternative diagnostic biopsy. Patients were informed of risks and benefits and alternatives to all procedures offered. All lesions acquired using an adhesive skin collection kit (DermTech, La Jolla, CA) according to package inserts. Standardized photography of clinical and dermoscopic findings were taken at each visit, and all lesions were followed for 3-months with short-term monitoring (STM) to ensure benign status. Negative PLA findings were excluded, positive findings were analyzed for histological changes using reflectance confocal microscopy (RCM) and shave biopsy depending on the patient's comfort level in STM. Two dermatologists reviewed histopathological features before arriving at a diagnosis. For patients quarantining due to the COVID-19 pandemic, photographs were obtained by patients using their smartphones with the DermLite HÜD ${ }^{\circledR}$ phone-adaptable dermatoscope. This retrospective 5-patient case series was exempt from institutional IRB approval.

Data Analysis: Patient information was collected from an electronic medical record system to an excel file, along with copies of the PLA findings, dermoscopic features, and the respective RCM 
report. Dermoscopic features associated with melanoma were recorded, including pattern, organization, color distribution. Lesions on RCM were analyzed for the presence of atypical cells, nests, organizational architecture, pattern, and features surrounding papillae at the dermo-epidermal junction (DEJ); features associated with melanomas.

Results: Five patients, ages 18-67 (median 31) presented with pigmented lesions measuring 4-9mm (median 6.5). PLA results were (5/5) + LINC -PRAME. All five lesions were imaged via RCM and one was biopsied due to the patient's discomfort with STM. Dermoscopy findings on 4/5 lesions showed small dark globules at the periphery of the lesion indicative of a lesion in a growing state, $1 / 5$ showed an atypical network with asymmetric hyperpigmentation. RCM findings showed (4/5) irregular or mixed honeycomb and cobblestone patterns in the epidermis with junctional and/or dermal melanocytic nests with mixed ringed or meshwork patterns. Few atypical melanocytes were seen mostly at the dermo-epidermal junction and no pagetoid cells were appreciated in the epidermis, consistent with mild to moderate dysplastic nevi. Histology on the biopsied lesion showed a rare sclerosing dysplastic nevus with whirled fibrosis surrounding melanocytic nests; additional cuts showed no mitotic division in the dermis. None of the lesions required re-excision. 3-month short-term monitoring showed no dermoscopic changes or signs that would otherwise require further management. This furthered the notion that +LINC -PRAME lesions were undergoing benign growth in otherwise benign, mild-moderately dysplastic nevi.

Conclusion: Our observations via RCM on +LINC00518-only lesions showed features of benign nevi undergoing subtle growth that are not worrisome for melanoma. While the most specific of the ABCDEs of melanoma is Evolution, this is usually only true with elderly patients, nevi in younger patients may undergo benign growth without progression to melanoma. Our findings show that LINC expression may play a role in the benign growth in atypical nevi, and thus do not warrant a biopsy. Current recommendations for lesions with positive findings on PLA is to biopsy $^{2}$ however RCM can be used to rule out melanoma, and along with short-term monitoring, can offer a safe alternative to traditional biopsies.

\section{References}

1. Ferris, LK, Rigel, DS, Siegel, DM, et al. Impact on clinical practice of a non-invasive gene expression melanoma rule-out test: 12-month follow-up of negative test results and utility data from a large US registry study. Dermatol Online J. 2019;25(5).

2. Ferris, LK, Gerami, P, Skelsey, MK, et al. Real-world performance and utility of a noninvasive gene expression assay to evaluate melanoma risk in pigmented lesions. Melanoma Res. 2018;28(5):478-482. doi:10.1097/CMR.0000000000000478

3. Lezcano, C, Jungbluth, AA, Nehal, KS, Hollmann, TJ, Busam, KJ. PRAME Expression in Melanocytic Tumors. Am J Surg Pathol. 2018;42(11):1456-1465. doi:10.1097/ PAS. 0000000000001134

4. Luan, W, Ding, Y, Ma, S, Ruan, H, Wang, J, Lu, F. Long noncoding RNA LINC00518 acts as a competing endogenous RNA to promote the metastasis of malignant melanoma via miR-204-5p/AP1S2 axis. Cell Death Dis. 2019;10(11):855. doi:10.1038/s41419-019-2090-3

$\star$ Poster No. *B7

Abstract No. B7

Category: Basic Science

Research Focus Area: Pain

Management

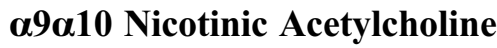

Receptor Function in

\section{Migraine-Related Pain}

Mehul M Shah, OMS IV ${ }^{1}$; J. Michael Mclntosh²;

Frank Porreca ${ }^{3}$; Jennifer Y. Xie

${ }^{1}$ New York Institute of Technology College of Medicine at Arkansas State University, Jonesboro, AR;

${ }^{2}$ Department of Biology, University of Utah, Salt Lake City, UT; ${ }^{3}$ Department of Pharmacology, University of Arizona - Health Sciences Center, Tucson, AZ; ${ }^{4}$ Department of Basic Sciences, New York Institute of Technology College of Medicine at Arkansas State University, Jonesboro, AR 
Statement of Significance: Migraine is one of the world's most common neurological disorder. Many individuals suffer from frequent headache limiting their productivity and diminishing their quality of life. Currently available migraine abortive drugs such as triptans, NSAIDs, and osteopathic manipulative techniques are not suitable, or effective, for everyone. For example, triptans are contraindicated for patients with cardiovascular risks due to their vasoconstrictive properties. In addition, all current acute medications provide satisfactory relief to less than $50 \%$ patients. Molecules with new mechanism of action and with improved tolerability and safety profiles represent an important clinical advance that could help many patients with episodic migraine.

Research Methods: Our participants were female $\mathrm{CBA} / \mathrm{CaJ}$ mice. Both wild-type and genetically altered mice with the $\alpha 9$ subunit of nAChRs eliminated, deemed $\alpha 9$ knockout mice, were utilized in our experiments. IACUC approval via Arkansas State University was achieved prior to conducting experiments. We employed a relatively new model of inducing migraines in wild type and $\alpha 9$ knockout mice to confirm the relevance of the proposed target. We utilized a functional tactile allodynia model in our experiments. Our control groups were injected with synthetic interstitial fluid (SIF - $\mathrm{pH} 7.4,5 \mu \mathrm{l}$ ) while experimental groups were injected with inflammatory mediators (IM - comprised of $1 \mathrm{mM}$ histamine, serotonin, bradykinin, and $0.1 \mathrm{mM}$ PGE2 at $\mathrm{pH} 5.0,5 \mu \mathrm{l})$. Injections were placed onto the dural surface through the skull sutures via a short injector without penetrating the dura. Response frequencies to probing of the periorbital and hindpaw regions using von Frey (VF) filaments 10 times were obtained before and after dural injections by an investigator blinded to the treatments to assess for cutaneous tactile allodynia. In subsequent experiments with our wild-type mice, we administered RgIA4 (500 $\mu \mathrm{g} / \mathrm{kg}$, s.c.) into select mice 30 minutes prior to the dural injections. We divided our mice into three groups for these experiments: one group with IM and Saline, another with SIF and RgIA4, and a third with IM and RgIA4.

Data Analysis: One-Way or Two-Way ANOVA and post hoc tests were carried out in Graphpad Prism 8.0. If data did not demonstrate normal distribution, equivalent non-parametric tests were applied. All results are represented as Mean \pm SEM.

Results: After injection of IM, the cutaneous tactile allodynia response frequency was $40+/$ $4.5 \%$ and $26+/-4.0 \%$ for periorbital and hindpaw tactile stimulus, respectively, in the wildtype mice. This was significantly increased from the naïve baseline of $7+/-2.7$ and $4+/-2.0 \%$, indicating robust cutaneous allodynia. Compared with their baseline of $7+/-1.8$ and $3+/-2.3 \%$, knockout mice showed only a $26+/-4.1$ and $20+/-7.1 \%$ increase of response frequency to the VF filaments, respectively, after IM injection. Our IM \& Saline group demonstrated a $32+/-2.2$ and $21+/-1.6 \%$ response frequency for the periorbital and hindpaw regions, respectively, increased from a baseline of $5+/-1.8$ and $2+/-1.2 \%$. The SIF \& RgIA4 group showed response frequencies of 13 $+/-1.5$ and $10+/-2.2 \%$ for the periorbital and hindpaw regions, respectively, compared with their baseline of $3+/-1.5$ and $2.5+/-1.4$. Our IM \& RgIA4 group demonstrated a $17+/-2.0$ and $15+/-1.6 \%$ response frequency for the periorbital and hindpaw regions, respectively, compared with a baseline value of $3+/-1.5$ and $2+/-1.2 \%$.

Conclusion: We have shown that blockade of $\alpha 9 \alpha 10$ nAchR via germ line elimination of the $\alpha 9$ subunit or via selective antagonist, RgIA4, inhibited cephalic and extracephalic pain in mouse migraine models induced by dural inflammation. Limitations to the experiments a relatively 
small sample size. Future research opportunities will explore the use of knockout mice in experiments utilizing RgIA4. These data support our hypothesis of $\alpha 9 \alpha 10 \mathrm{nAChR}$ antagonists representing a viable mechanism for acute treatment of migraine-related pain.

\section{References}

1. Adelman, JU, Belsey, J. Meta-analysis of oral triptan therapy for migraine: number needed to treat and relative cost to achieve relief within 2 hours. J Manag Care Pharm. 2003 Jan-Feb;9 (1):45-52.

2. Burgos-Vega, CC, Quigley, LD, Trevisan Dos, Santos G, et al. Non-invasive dural stimulation in mice: A novel preclinical model of migraine. Cephalalgia. 2018 Jan 1:333102418779557.

3. McGregor, EA. In the clinic. Migraine. Ann Intern Med. 2013 Nov $5 ; 159(9)$

4. Shelukhina, I, Mikhailov, N, Abushik, P, Nurullin, L, Nikolsky, EE, Giniatullin, R. Cholinergic Nociceptive Mechanisms in Rat Meninges and Trigeminal Ganglia: Potential Implications for Migraine Pain. Front Neurol. 2017;8:163.

5. Romero, HK, Christensen, SB, Di Cesare Mannelli L, et al. Inhibition of alpha9alpha10 nicotinic acetylcholine receptors prevents chemotherapy-induced neuropathic pain. Proc Nat Acad Sci U S A. 2017 Mar 07;114(10):E1825-E32.

Poster No. *B15

Abstract No. 15

Category: Clinical

Research Focus Area: Chronic Diseases \& Conditions

\section{COVID-19 and Obesity in Younger} Adults: A Systematic Literature

\section{Review}

Emma Michelle Griffith, OMS IV ${ }^{1}$; JuliSu Dimucci-Ward, PhD, MPH, RD, CDE, LD 2 . Amber Stroupe, DO ${ }^{1}$

${ }^{1}$ Edward Via College of Osteopathic Medicine (South Carolina), Spartanburg, SC; ${ }^{2}$ Departments of Preventative Medicine and Public Health, Edward Via College of Osteopathic Medicine (South Carolina), Spartanburg, SC

Statement of Significance: The COVID-19 pandemic has changed the world and urgently requires a deeper look into risk factors related to adverse outcomes so that the medical community may risk stratify patients and the population may proactively work to limit risk. Obesity is a prevalent condition, and has been associated with worse outcomes in other respiratory viral illnesses like influenza. The adult population younger than sixty-five years of age has been affected more than anticipated, despite lower prevalence of the co-morbidities found in older populations. Assessing COVID-19 patients holistically, structural variation such as obesity influences function via inflammation, respiratory ability, and other physiologic mechanisms- thus influencing prognosis.

Research Methods: Using PubMed, Google Scholar, and Helioblast, an online search was completed based on the following keywords: COVID-19 and obesity, BMI and COVID-19, COVID-19 risk factors, and young adults COVID-19. They were evaluated in order, based on relevance, and collected into citation manager EndNote. Inclusion criteria for the articles are the following: published in 2019-2020, COVID-19 positive study population, human study population, and an adult patient population (age 18+). Articles were required to be peer-reviewed and accepted for publication.

Data Analysis: Multiple studies have demonstrated that obesity is a risk factor for more severe clinical course of COVID-19 in patients younger than 65 years of age.

One study by Lighter et al. is frequently mentioned by other articles, as it is one of the few studies that specifically looked at obesity and COVID-19 in younger patients. Another study described an inverse correlation between age and body mass index, with younger age and higher BMI leading to worse outcomes in patients with 
COVID-19. A table plotting age and BMI by researchers in Spain noted that increasing age and $\mathrm{BMI}>25 \mathrm{~kg} / \mathrm{m} 2$ were represented disproportionately in patients admitted to the ICU with COVID-19; however, almost half of those patients were between 25-65 years old. Additionally, a retrospective cohort study on patients in New Jersey showed that obesity was an independent risk factor for adverse outcomes including hospital admission, use of mechanical ventilation, and death in patients between 18-45 years of age with COVID-19.

In a case report, an obese patient in their 30's with comorbidities of hypertension and diabetes mellitus experienced an internal carotid artery occlusion secondary to the hypercoagulable state from COVID-19. The four patients with cerebrovascular accidents and COVID-19 in that case series had an average age of 52.8. A meta-analysis confirmed that obese adults with COVID-19 had a more severe outcome overall, but did not further elaborate on adults younger than 65 vs older than 65 .

Results: Overall, studies showed that obesity is a risk factor for severe COVID-19 infection in adult patients younger than sixty-five years, specifically requirement of mechanical ventilation as the marker of severe clinical course. The prothrombotic state associated with COVID-19 has led to cerebrovascular accidents in patients younger than fifty years of age.

COVID-19 pathogenesis includes a cytokine storm in severe cases, and obese patients already have high baseline levels of inflammatory cytokines such as interleukin-6 (IL-6) and tumor necrosis factor alpha (TNF $\alpha)$. Another literature review by Banerjee et al. stated that the pre-existing inflammatory state associated with obesity explains the worse outcomes of patients, regardless of age.
Further studies will be incorporated into this review in order to provide the most up-to-date information.

Conclusion: It has become widely accepted that obesity is associated with increased severity of COVID-19 clinical course in older adults. However, there is a small portion of research demonstrating that obesity can increase an individual's risk of a more severe clinical course, as defined by ICU admission, requirement of mechanical ventilation, or death, of COVID-19 in patients younger than sixty-five years of age.

Higher levels of inflammatory mediators like interleukin- 6 in obese patients may explain the hyper-inflammatory response and worse prognosis of COVID-19 in that population, among other physiologic mechanisms such as reduced immunity secondary to poor nutrition, insulin resistance, reduced respiratory function, and increased angiotensin-converting enzyme-2 (ACE2) receptor expression.

Additional research focused on anthropometric and metabolic parameters associated with worse COVID-19 disease course is needed. Establishing obesity as a factor influencing COVID-19 severity in younger adults helps with risk stratification moving forward in this pandemic.

Acknowledgment/Funding Source: First and foremost, I would like to thank Dr. Dimucci-Ward for her constant guidance and encouragement throughout this process. I would also like to recognize Bill Nichols at VCOM for his assistance in the literature search process. Finally, thank you to Dr. Amber Stroupe for providing edits and advice in order to transform this paper. There are no funding sources to disclose. 


\author{
References \\ 1. Banerjee, Mithu, et al "Obesity and Covid-19: A Fatal Alliance." \\ 2. Calder, Philip C., et al CDC. "Preliminary Estimates of the \\ Prevalence of Selected Underlying Health Conditions among \\ Patients with Coronavirus Disease 2019 - United States, \\ February 12-March 28, 2020." MMWR. \\ 3. CDC. "Severe Outcomes among Patients with Coronavirus \\ Disease 2019 (Covid-19) - United States, February 12-March \\ 16, 2020." \\ 4. Fernández García, L., A.B. Puentes Gutiérrez, and M. García \\ Bascones. \\ 5. Kass, David A., Priya Duggal, and Oscar Cingolani. \\ 6. Lighter, J., et al \\ 7. Petrilli, Christopher et al \\ 8. Scheen, A. J. \\ 9. Stefan, N., et al \\ 10. Steinberg, Eric, Ellsworth Wright, and Beth Kushner \\ 11. Wang, A., et al \\ 12. Watanabe, M., et al \\ 13. Yang, J., J. Hu, and C. Zhu. "Obesity Aggravates Covid-19: \\ A Systematic Review and Meta-Analysis."
}

$\star$ Poster No. *B20

Abstract No. 20

Category: Clinical

Research Focus Area: Chronic

Diseases \& Conditions

Efficacy of Green Tea Consumption on

Weight Loss and Maintenance: $A$

Meta-Analysis

Kaiming Tan, OMS II ; Marcelle Savoy, $\mathrm{PhD}^{2}$; Lori McGrew, $\mathrm{PhD}^{3}$; Lauren Wisnieski, MS PhD Karen Gruszynski, DVM, MPH, PhD, DACVPM ${ }^{4}$; Mary Beth Babos, MS PharmD BCPS ${ }^{2}$

${ }^{1}$ Lincoln Memorial University - DeBusk College of Osteopathic Medicine, Knoxville, TN; ${ }^{2}$ Lincoln Memorial University - DeBusk College of Osteopathic Medicine Reed Health Sciences Library, Knoxville,

TN; ${ }^{3}$ Department of Pharmacology, Lincoln Memorial University - DeBusk College of Osteopathic Medicine, Harrogate, TN; ${ }^{4}$ Lincoln Memorial University College of Veterinary Medicine Center for Animal and Human Health in Appalachia, Harrogate, TN

Statement of Significance: To our knowledge, this is the first meta-analysis to assess recently published RCTs that explore green tea's impact on weight-related parameters. Obesity is a worldwide concern with significant impact on health care costs and chronic morbidities such as cardiovascular disease and diabetes (Jurgens et al., 2012). The Osteopathic approach considers aspects of lifestyle that impact disease risk. Addition of green tea is a simple dietary change that may reduce the risk of chronic diseases associated with obesity. Our meta-analysis assesses the potential of green tea in weight loss and maintenance. The results provide updated guidance on dietary recommendations and offer insight into effect size translatable to the general population.

Research Methods: We formulated our research question using the PICO framework: $\mathrm{P}=$ nonpregnant adults; $\mathrm{I}=$ consumption of green tea or green tea extract supplement over four weeks or more; $\mathrm{C}=$ green tea vs control; $\mathrm{O}=$ change in weight-related parameters including absolute weight (kg), Body Mass Index (BMI) (kg/m2), and/or Waist Circumference (WC) (cm). For this meta-analysis, a search was conducted for relevant studies in MEDLINE, ClinicalTrials.gov, CINAHL, TRIP, Cochrane Central of Controlled Trials and Science Direct Health and Life Sciences. Restrictions were applied to English language only investigations. Inclusion criteria comprised of parallel-design, randomized, double-blind, controlled trials that directly examined whole or decaffeinated green tea or green tea extract effect on body weight, BMI, or WC in non-pregnant adult humans. Studies with experimental arms that involved ingestion of active ingredients combined with green tea were excluded. By combining our selected studies, we created a large and varied pool of human participants so that the results could be readily applied to nutritional advisories for the general population. 
Data Analysis: Two authors independently reviewed articles for inclusion with differences resolved by consensus. Three authors independently assessed studies to assign risk of bias scores. The mean for each of six domains was calculated to assign a joint risk of bias assessment. Data reflecting changes in absolute body weight, body mass index (BMI), waist circumference (WC), and \% change in body fat were extracted independently by two authors. Data extracted from included studies represented multiple varied populations exposed at varying doses for varying lengths of time, thus a random effects model was selected for analysis. Heterogeneity of studies was evaluated through use of forest plots and the I2 statistic using RevMan5 software (Review Manager 2020). Publication bias was assessed using a funnel plot. For studies with multiple intervention arms, a decision was made a priori to calculate mean results for interventions that included green tea or green tea extract as active and those that did not include green tea or extract as control. When necessary, data of varying measurement units were synthesized by meta-analysis and analyzed using $\mathrm{R}$ ( $\mathrm{R}$ Foundation for Statistical Computing).

Results: After database search and screening, fifteen studies were included for meta-analysis. Populations included for study, duration of study, dosing conditions, total daily dose of green tea or extract, caffeine content of treatments, and measurements to assess change in body composition were not homogenous. Daily doses of green tea ranged from $379 \mathrm{mg}$ standardized extract to $20 \mathrm{mg}$ green tea solids. Duration of interventions ranged from four to forty-eight weeks. Upon completion of data analysis, all results will be reported.

Conclusion: Studies that were included in our meta-analysis were widely varied in patient selec- tion, dosing, dosing strategy, and outcome measurements. Information gained from our analysis, once complete, can help determine feasibility of future studies. An estimate of effect size from such varied methodologies can similarly help determine sample size needed for further studies. The wide variation in studies may reflect more closely how green tea consumption in real life impacts actual weight related parameters.

There are many knowledge gaps that remain to be investigated. For example, factors such as biological sex, ethnicity, and pre-existing conditions have an impact on the efficacy of green tea in weight reduction and maintenance. A limitation of our study is that some of the RCTs we selected did not document the ethnicity of participants. It has been shown that the genetic makeup of an individual may be a confounding factor in green tea's effects on weight loss. Future research opportunities include conducting large RCTs to address the public health concern of obesity and preventive medicine. If our results show a reasonable effect size, lifestyle modifications that incorporate green tea may offer a tool consistent with Osteopathic Principles to promote patient wellness through impact on body weight.

Acknowledgment/Funding Source: The authors would like to thank Dr. Craig Warden (Professor of the Department of Neurobiology, Physiology and Behavior at University of California-Davis) for inspiring an interest in this topic.

\section{References}

1. Jurgens, T., Whelan, A., Killian, L., Doucette, S., Kirk, S. and Foy, E., 2012. Green tea for weight loss and weight maintenance in overweight or obese adults. Cochrane Database of Systematic Reviews, (12).

2. R statistical software [Computer program]. R Core Team. Vienna, Austria: R Foundation for Statistical Computing, 2013. URL http:// www.R-project.org/.

3. Review Manager (RevMan) [Computer program]. Version 5.4, The Cochrane Collaboration, 2020. 
Poster No. *B23

Abstract No. 23

Category: Health Services

Research Focus Area: Osteopathic Philosophy

\section{A Pilot Study on the Usability of Step Trackers to Measure Physical Activity in Children}

Erin R. Christensen, OMS IV ${ }^{1}$; John C. Efstathiades, OMS IV ${ }^{1}$; Benjamin D. Tift, OMS IV ${ }^{1}$; Alexis

M. Stoner, $\mathrm{PhD}, \mathrm{MPH}^{2}$; Hanna S. Sahhar, MD ${ }^{3}$

${ }^{1}$ Edward Via College of Osteopathic Medicine (South

Carolina), Spartanburg, SC; ${ }^{2}$ Discipline Chair for

Epidemiology,Community and Public Health and

Preventive Medicine, Edward Via College of

Osteopathic Medicine (South Carolina), Spartanburg,

SC; ${ }^{3}$ Discipline Chair for Pediatrics, Edward Via

College of Osteopathic Medicine (South Carolina),

Spartanburg, SC

Statement of Significance: The PLAY Program was developed to increase physical activity levels in students of low socioeconomic status. Previously, step tracking devices were used to measure the effectiveness of the PLAY Program. While these devices have been shown to provide valid measurements of physical activity in adults and short-term in children, there is a lack of research demonstrating effectiveness and adherence long-term in the pediatric population. This study is significant due to its potential to inform osteopathic efforts to improve pediatric population health through the evaluation of methods to objectively measure physical activity in children.

Research Methods: The PLAY Program is a bi-monthly, after school program for third grade students at a low socioeconomic status charter school that incorporates exercise activities, teamwork, and education on topics including nutrition, exercise, health, and overall well-being. Third grade students enrolled at a school where PLAY sessions were conducted were given step tracking devices. Those children who attended PLAY were included in the intervention group and all others were invited to participate in the control group. Students were given a Garmin Vivofit 3 activity tracker and were asked to wear it continuously. Data from the devices was collected weekly. A standardized open-ended questionnaire was used to engage students in interviews regarding their perceptions of the step trackers. The questionnaire was developed from a similar study and focused on students' likes and dislikes regarding step trackers, how step trackers changed students' physical activity levels, and students' perceptions on how the program could be improved.

Data Analysis: Descriptive statistics were performed to compare the students enrolled in the PLAY group and the control group. Interviews were qualitatively analyzed independently by three researchers using thematic coding. Researchers identified common elements of participants' experiences with the step trackers. Codes were reviewed by all researchers for possible connections, resulting in themes. Themes were determined by iterative review of text.

Results: Of the 14 participants in the first phase of the study, nine were enrolled in the PLAY Program and five were enrolled in the control group. Two students (one in the PLAY Program and one in the control group) lost their device before data collection began. When device data collection was stopped, $43 \%$ of students had no recorded activity over the previous 4 weeks, and $50 \%$ of the students who had data within the previous 4 weeks had recorded less than 7 days of data. At the conclusion of data collection, the average number of steps per day for students enrolled in the PLAY Program was 2609.56 (95\% CI: 2377.35-2841.77) compared with 1157.95 (95\% CI 914.65-1401.26) for students in the control group. 
Of the 16 students who were given step trackers, 11 agreed to participate in the qualitative portion of the study. Six of these students (55\%) lost their device. Three central themes emerged. 83\% of students who reported a lost step tracker reported that the device had fallen off their wrist in a public place. $36 \%$ of students found locks on the bands of the devices to be frustrating and ineffective. $73 \%$ of students felt the goal setting feature on the device motivated them to exercise more, while $55 \%$ of students specifically cited the goal setting feature as the main motivator for exercising more. $40 \%$ of students who still had their devices and continued to wear them because they still enjoyed these goal-setting features.

Conclusion: Although the PLAY group recorded a higher average of steps over the course of data collection, this number should be interpreted with discretion due to the small sample size and a large amount of missing data. Unless researchers have a reliable way of ensuring compliance, step trackers should not be relied on for an accurate measure of physical activity in children. This study has several limitations. This study has a relatively small sample size. Our results only examined students' perceptions regarding one device and may not represent how children with other step tracking devices feel about them. Recall and response biases were also likely factors in this study, as many students had gone several months between losing their step tracker and the qualitative interviews, and because students were interviewed by the three researchers who led PLAY Program sessions. Since the major barrier encountered in this study was relying on participants to maintain the device on their wrists to ensure accurate data was collected, the possible solutions discussed here may help researchers overcome this barrier in the future.

Acknowledgment/Funding Source: Supplies for this project were funded by Edward Via College of Osteopathic Medicine.
Poster No. *B28

Abstract No. 28

Category: Clinical

Research Focus Area: Osteopathic Philosophy

AOA Grant Number: 2851807727

\section{Burn-out in Osteopathic Medical Students: The Effects of Meditation Before and After the Arrival of the COVID-19 Pandemic}

Bhuma Krishnamachari, PhD ${ }^{1}$; Alex Morris, OMS III'; Ravi Chinsky, OMS III²; Ramya Pendyala, OMS II; William Blazey, DO ${ }^{4}$; Jerry Balentine, DO ${ }^{1}$ ${ }^{1}$ New York Institute of Technology (NYIT), Old Westbury, NY; ${ }^{2}$ Academic Medicine Scholars Program, New York Institute of Technology (NYIT), Old Westbury, NY; ${ }^{3}$ Department of Research, New York Institute of Technology (NYIT), Old Westbury, NY; ${ }^{4}$ Department of Academic Affairs, New York Institute of Technology (NYIT), Old Westbury, NY

Statement of Significance: According to the American Association of Colleges of Osteopathic Medicine (AACOM), DOs are trained to look at people holistically from the inception of medical school. Decreasing burnout in osteopathic medical students is particularly important as they are the future of the health care workforce. Research shows that physician burnout is directly connected with stress that is initiated during medical school and then continues forward. Thus, addressing stress at an early stage in physician training is critical. While there is some research that shows an association between meditation and general stress levels, few studies have examined the effect of meditation specifically on burnout, which is a term reserved for work related stress.

Research Methods: As a part of a larger IRB approved longitudinal randomized controlled trial, first, second and third year osteopathic medical students were recruited and randomized 
into an intervention group (use of the Headspace meditation app or control group (no use of the app). For this analysis, only students who entered the study in July 2019 were included, since this cohort uniquely experienced the COVID-19 pandemic (beginning January 21, 2020 in the U.S.). The analysis included 15 students in the intervention group and 14 controls. Students in the intervention groups used the app 3 days a week, 10 minutes each session. Burn-out was measured prior to study initiation, 3 months, 6 months (prior to the pandemic beginning) and 9 months (mid-April, at the peak of the pandemic in New York) using the Maslach Burnout Inventory (MBI). The MBI is a validated and widely used survey tool that evaluates the three types of feelings comprising burnout: namely exhaustion, cynicism and low professional efficacy. Each aspect of burnout is scored between 0 and 6 , with higher scores indicating higher degrees of burnout for the exhaustion and cynicism measures, and lower scores indicating higher degrees of burnout for the professional efficacy measure.

Data Analysis: All surveys were sent to students using the RedCap data collection and management system. For analysis, data was extracted into an excel spreadsheet and analysis was conducted using SAS 9.4 software. All patients were analyzed using intention-to-treat methods. $\mathrm{T}$ tests were used to compare the intervention group and control groups on all continuous variables at each time point. Subgroup analyses were conducted according to self-reported sex. Specifically, subgroup analyses were performed separately on 15 females and 12 males. A p-value under 0.05 was considered statistically significant.

Results: At baseline and at three months, the intervention and control groups did not differ in exhaustion, cynicism or professional efficacy. However, subgroup analyses showed that in females, there was a statistically significant difference $(p=0.02)$ between the control group $($ mean $=3.75$, SD 1.11) and intervention group (mean=2.40, SD 0.75) in cynicism at three months, but the same difference was not seen in males. At six months, there was a significant difference between groups in cynicism, with the mean score in controls at 3.90 (SD 1.35) and the mean score in the intervention group at 2.93 (SD 1.09), $(p=0.04)$. With subgroup analysis at six months, in females, there was a statistically significant difference in terms of exhaustion $(p=0.002)$ between the control group $($ mean $=5.48, \mathrm{SD} 0.93)$ and intervention group (mean=3.63, SD 0.91), and a borderline but not significant $(\mathrm{p}=0.05)$ difference between groups in cynicism((control mean=4.10 (SD0.95), intervention mean=2.94 (SD1.12)). At nine months, about 45 days after the COVID-19 pandemic had arrived in April 2020, no statistically significant differences were seen in any aspect of burn-out in between the intervention and control groups. Subgroup analysis demonstrated just one statistically significant difference: this was in females for the exhaustion measure (control group mean $=5.27(\mathrm{SD} 1.34)$, intervention mean $=3.60$ $\mathrm{SD}$ (1.28), $\mathrm{p}=0.04)$.

Conclusion: This study found that meditation using the Headspace app may be effective for improving burn-out levels among osteopathic medical students, particularly amongst female medical students. This is consistent with existing research that suggests that there are differences between genders in terms of burn-out. Females seemed to benefit from the meditation app at an earlier time in terms of cynicism. By six 
months, there was benefit seen from the meditation app in terms of cynicism in the whole student population, and in females, there also seemed to be a benefit in terms of exhaustion. However, any significant differences between the groups in terms of cynicism were eliminated when the students were surveyed at the height of the pandemic, though in females the significant difference in exhaustion between groups remained. This suggests that meditation protocols may need to be adjusted for different individuals and for different circumstances. Acute and intense stressors such as the pandemic may require interventional protocols beyond what is used for day to day stressors for a medical student. Future analyses will consider additional pertinent information such as demographic details. If ongoing research shows that medical student burn-out decreases with use of a meditation app, medical schools may choose to incorporate this or other tools geared towards stress reduction in their wellness curriculums.

Acknowledgment/Funding Source: Funding for this pilot study was provided by AOA grant number 2851807727

\section{References}

1. Bond, AR, Mason, HF, Lemaster, CM, Shaw, SE, Mullin, CS, Holick, EA, Saper, RB. Embodied health: the effects of a mind-body course for medical students. Med Educ Online. 2013 Apr 30;18:1-8.

2. Victor, J., Dzau, M.D. Darrell G. Kirch, M.D., and Thomas J. Nasca, M.D. To Care Is Human - Collectively Confronting the Clinician-Burnout Crisis. N Engl J Med 2018; 378:312-314 DOI: 10.1056/NEJMp1715127.

3. Kötter, T, Wagner, J, Brüheim, L, Voltmer, E. Perceived Medical School stress of undergraduate medical students predicts academic performance: an observational study. BMC Med Educ. 2017; 17(1):256 .

4. Worly, B, Verbeck, N, Walker, C, Clinchot, DM. Burnout, perceived stress, and empathic concern: differences in female and male Millennial medical students. Psychol Health Med. 2018;:1-10.
Poster No. *B30

Abstract No. 30

Category: Basic Science

Research Focus Area: Chronic

Diseases \& Conditions

\section{Investigation of Intensity Dependent}

Transcranial Direct Current

Stimulation (tDCS) Used in the

Therapy for Cerebellar Ataxia: An

\section{In-Vivo Approach}

Prakshal Jain, OMS $\|^{1}$; Salvatore C. Jerris, OMS II

George K. Mathew, OMS II ; Huo Lu, PhD ${ }^{2}$

${ }^{1}$ Philadelphia College of Osteopathic Medicine

(Georgia Campus), Suwanee, Georgia; ${ }^{2}$ Bio-Medical

Sciences, Philadelphia College of Medicine -

Suwanee, Georgia Campus, Suwanee, Georgia

Statement of Significance: Osteopathic medicine focuses on a holistic approach to treat patients that encompasses not only anatomy but also the function and behavior of the patient. Our study investigates one aspect of the mechanisms behind tDCS effects on the cerebro-cerebellar circuit. This method has been repeatedly suggested to treat movement disorders stemming from the cerebellum such as cerebellar ataxia [1]. Investigating the intensity of tDCS in the regulation of Purkinje cells along with the correlative effect on local field potentials (LFP) between the Purkinje cells and motor cortical LFP may lead to a better understanding on the possible mechanisms of tDCS and its optimal application.

Research Methods: Cranial surgical procedures were performed on three adult Sprague-Dawley rats [2]. Induction of anesthesia began with the administration of isoflurane in a closed induction chamber followed by weight-based administration of a Ketamine/ Xylazine/ Acepromazine cocktail. Vitals were measured throughout the surgical experiment by means of a live rectal temperature 
probe and ECG leads to monitor heart rate. Supplemental cocktail injections were regularly administered with immediate dosage given following the return of a pedal reflex, assessed every fifteen minutes. The rats were immobilized using a stereotaxic apparatus and surgery was performed to expose areas of both the cerebellar and motor cortices to allow for penetration of the recording electrodes. Purkinje cell isolation was determined based on the depth of the electrode, the firing pattern, and the complex spike traces with live trace readings [2]. The recording ground electrode was placed under the skin superior to the skull and anteriorly to the incision and the stimulation ground electrode was guided through the cheek. The stimulation electrode was positioned on the skin immediately posterior to the exposed cerebellar surgical site. Direct current was delivered from 50 to $300 \mu \mathrm{A}$ for five minutes in $50 \mu \mathrm{A}$ increments. Neural recordings were collected in three segments: before stimulation (control), during stimulation, and after stimulation (post-stimulatory control). The data were then analyzed using MATLAB.

Data Analysis: Data analysis was performed using a mathematical software program, MATLAB (R2020a). The data was filtered between 1-10,000 Hz. Average firing rates of the Purkinje cells were calculated to determine the change at different stimulation intensities to study the direct effect of tDCS. For each data set, a power spectrum plot was generated to display dominant frequency changes of LFP recorded from the cerebellar and motor cortices before, during, and after stimulation. Plots demonstrating cross-correlation and coherence between cerebellar and motor cortical activity were also generated for recordings from before, during (50-300 $\mu \mathrm{A}$, $\Delta=50 \mu \mathrm{A}$ ), and after stimulation for each set of data. This data analysis is to explore the consequent effects of a range of stimulation intensities on the cerebello-thalamo-cortical pathway.
Results: The average Purkinje cell firing rate was variable across all $(n=4)$ data sets. An overall intensity-dependent decrease of Purkinje cell firing rate was observed, with an increase in Purkinje cell firing rate during the 100-150 $\mu \mathrm{A}$ stimulation levels when compared with the pre-stimulatory control. The cerebellar power spectrum analysis showed no clear changes at low frequencies when comparing each stimulation level to the control. However, an increase in peak amplitude was seen at $9 \mathrm{~Hz}$ during the $50 \mu \mathrm{A}$ stimulation in 2/4 recordings as compared with the control. The same frequency of $9 \mathrm{~Hz}$ was further examined in the coherence analysis. 2/4 recordings showed decreased coherence after $50 \mu \mathrm{A}$ whereas the other $2 / 4$ showed no pattern in coherence. The cross-correlation level between the cerebellum and motor cortical LFPs showed intensity-dependent changes $50-300 \mu \mathrm{A}$. 3/4 of the recordings showed an increased cross-correlation while $1 / 4$ showed a decreased cross-correlation

Conclusion: Based on preliminary data, intensitydependent changes were observed in the motor cortex and cerebellum. The average firing rate of the Purkinje cell decreased during stimulation when compared with the post-stimulation tDCS. The increase in cross-correlation between the cerebellar and motor cortices indicates these two brain regions become more associated with increased tDCS intensity. Limited intensity-dependent findings were observed in the power spectrum and coherence analysis at $9 \mathrm{~Hz}$ however, changes could have occurred at other frequencies. Overall, the decreased Purkinje cell firing rate and increased cross-correlation indicate the tDCS intensity stimulation under 150-200 $\mu \mathrm{A}$ generated increased output from the cerebellum to the motor cortex. This increased output through the cerebello-thalamo-cortical pathway can affect gross motor functions. Further research is needed to confirm changes observed at these stimulation intensities. The findings of tDCS intensity in this study 
can be applied proportionally in the investigation of optimal therapies for patients with cerebellar ataxia.

Acknowledgment/Funding Source: We would like to thank Ms. Shaunda Bell for training at the PCOM Georgia campus animal facility. We also thank our CSO for funding this research.

\section{References}

1. Ferrucci, R., Bocci, T., Cortese, F. et al. Cerebellar transcranial direct current stimulation in neurological disease. cerebellum ataxias 3, 16 (2016). https://doi.org/10.1186/s40673-016-0054-2

2. Bower, JM, Beermann, DH, Gibson, JM, Shambes, GM, Welker, W (1981) Principles of organization of a cerebro-cerebellar circuit. Micromapping the projections from cerebral (SI) to cerebellar (granule cell layer) tactile areas of rats. Brain Behav Evol 18:1-18.

Poster No. *B37

Abstract No. 37

Category: Basic Science

Research Focus Area: Chronic

Diseases \& Conditions

Sequence and Structural Analysis of Kinases in the mTOR Pathway to Evaluate Their Function Involved in SARS-CoV-2 Replication

Taylor Barr, OMS II ; Crystal Boudreaux, $\mathrm{PhD}^{2}$ ${ }^{1}$ West Virginia School of Osteopathic Medicine, Lewisburg, WV; ${ }^{2}$ Department of Biomedical Sciences, West Virginia School of Osteopathic Medicine, Lewisburg, WV

Statement of Significance: Protein sequence and structure analysis to identify regions that function in facilitating viral replication is a direct application of the osteopathic tenet of structure and function interdependence. Elucidating which areas of the previously mentioned host cell proteins are involved in facilitating viral replication will be key to targeted design of anti-viral drugs. Identifying drug targets common to RNA virus replication pathways would allow for effective treatments and possible prophylaxis that would not only aid physicians in the current public health crisis involving SARS-CoV-2, but also potentially prepare health care providers to manage future novel RNA virus outbreaks until an effective vaccine is developed.

Research Methods: The canonical amino acid sequences for each of the proteins (STK11IP, LARP1, CK2, and mTOR) were obtained from Universal Protein Resource (UniProt), and then each of the protein sequences were used in the National Center for Biotechnology Information's protein basic local alignment search tool. Sequence alignments from the query and their homologs across different species were examined to identify highly conserved residues, signifying regions of protein important to structure and function, as well as unique features between homologs. Additionally, protein sequences were imported from UniProt into SnapGene Viewer to identify previously determined domains within each protein. The protein sequences were also used as queries within the Class, Architecture, Topology, and Homology database website to identify homologous/related structural motifs and sequences. A consensus sequence was identified from those families and compared with the sequence of each protein to search for possible novel functions that may hint at how these host cell proteins facilitate viral replication. These sequences of interest were identified and highlighted in the molecular modelling software UCSF Chimera for each host cell protein with an elucidated structure to further investigate plausibility of the sequence/structure in facilitating host cell and virus protein-protein interaction.

Data Analysis: An amino acid residue was considered conserved if it was the same throughout all homologs at a particular alignment position or if there were only 'positive' substitutions at that 
alignment position as determined by the National Center for Biotechnology Information's protein basic local alignment search tool. Percent conservation of a protein was determined as the ratio of conserved residues in a protein to the total number of residues within that protein. Amino acid residues were categorized based on charge properties (positive, negative, polar neutral, and non-polar neutral), and trends or patterns were noted and documented using Excel. Sequences of interest were selected and highlighted on each protein's structure imported from a Protein Database file into Chimera. The highlighted structures were then evaluated based on whether they were on the surface or buried within the folded protein.

Results: Preliminary results show STK11IP is $50 \%$ conserved across the species in the alignment, and mTOR is remarkably conserved with a high fidelity of $95 \%$ across the species in the alignment. Alignment analysis is still ongoing for both these proteins as well as CK2 and LARP1. The Class, Architecture, Topography, and Homology database proposed STK11IP homology matches with six different structural domains (all parallel beta sheets) and with 38 different functional families. Of note among the proposed homologous functional families were ribonuclease inhibitor 1 and Toll-like receptor 7 because of their function of preventing RNA degradation and facilitating intra-cellular pathogen immune response respectively. All suggested homology from the Class, Architecture, Topography, and Homology database was toward the N-terminus, and there was no homology toward the C-terminus. There were (to date) two regions of interest, one within STK11IP and one within mTOR, that may warrant further investigation in vitro. Structural analysis is still ongoing for all proteins, particularly CK2 and LARP1.

Conclusion: This analysis will provide a preparatory base for future confirmatory experiments that will identify potential therapeutic targets and allow for targeted anti-viral drug design. This is important because of the shortage of effective drugs in treating patients who have contracted COVID-19 as well as lack of prophylaxis. It may help to develop more types of anti-viral therapies that will be effective at treating and preventing COVID-19, as well as for RNA viruses at large. Because a solved crystal or cryo-electron microscopy (cryo-EM) structure has not been elucidated for STK11IP, mapping the sequences of interest to a structure was not possible. In order to properly examine the identified sequences in terms of their corresponding structure as candidate sites for enhancing viral replication, we suggest either $\mathrm{x}$-ray crystallography or cryo-EM imaging of STK11IP to solve the protein structure as a future direction for this work.

This work is funded by the West Virginia Clinical and Translational Science Institute (NIH/NIGMS Award Number U54GM104942)

\section{References}

1. Kindrachuk, J, Ork, B, Hart, BJ, et al. Antiviral potential of ERK MAPK and PI3K/AKT/mTOR signaling modulation for Middle East respiratory syndrome coronavirus infection as identified by temporal kinome analysis. Antimicrob Agents Chemother 2015;59(2):1088-1099. doi:10.1128/AAC.03659-14

2. Protein [Internet]. Bethesda (MD): National Library of Medicine (US), National Center for Biotechnology Information; [1988] [cited 202007 13]. Available from: https://www.ncbi.nlm.nih.gov/ protein/

3. Ian Sillitoe, Natalie Dawson, Tony E Lewis, Sayoni Das, Jonathan G Lees, Paul Ashford, Adeyelu Tolulope, Harry M Scholes, Ilya Senatorov, Andra Bujan, Fatima Ceballos Rodriguez-Conde, Benjamin Dowling, Janet Thornton, Christine A Orengo. Nucleic Acids Res. 2019 Jan Pubmed: 30398663 doi: 10.1093/nar/gky1097 
$\star$ Poster No. *B45

Abstract No. 45

Category: Basic Science

Research Focus Area: Chronic

Diseases \& Conditions

\section{Mutation of Polycystin in ADPKD is Implicated in Aberrant Skeletal Muscle Phenotype in Zebrafish.} Alissa Frances Schurr, OMS III, AMS ${ }^{1}$; Sarah Raza, $\mathrm{DO}^{2}$; Ying $\mathrm{Li}^{2}$; Aleksandr Vasilyev, $\mathrm{MD}, \mathrm{PhD}^{2}$ ${ }^{1}$ New York Institute of Technology (NYIT) Old Westbury, NY; ${ }^{2}$ Department of Biomedical Sciences, New York Institute of Technology (NYIT), Old Westbury, NY

Statement of Significance: Autosomal dominant polycystic kidney disease (ADPKD) is a ciliopathic condition resulting from mutations in the polycystin genes, PKD1 and PKD2. This disease manifests as prominent fluid-filled renal cysts and other extrarenal abnormalities. Zebrafish models of ADPKD develop the expected cystic morphology, but also develop a peculiar dorsal axis curvature (DAC) of the tail, the significance of which remains undetermined. This study aimed to further elucidate the nature of DAC in PKD mutant and morphant zebrafish through analysis of dorsal myotomes and birefringence patterns. Osteopathically, the tenet of reciprocal interrelation of structure and function guided our decision to look beyond the kidneys, toward the neuromusculoskeletal system.

Research Methods: ET(krt8:EGFP)sqet11-9 transgenic zebrafish embryos at the 1-2 cell stage were microinjected with renal cyst-inducing polycystin-2 morpholino. These embryos were imaged at 1,2, and 8 days post-fertilization (dpf) to record myotome morphology. Confocal microscopy and imaging of GFP and DAPI stained fish were performed on PKD2 morphants and controls at $2 \mathrm{dpf}$; nuclei in ventral and dorsal myotome segments were quantified and correlated with myotome length. To assess the effects of PI3K inhibition on DAC, embryos were treated with LY294002 (LY; PI3K inhibitor) for 24hrs starting at $1 \mathrm{dpf}$; segment and trunk lengths were subsequently measured. Interactions between polycystin and PI3K were analyzed by exposing morphant PKD2 fish to LY from the 25-somite stage through $3 \mathrm{dpf}$. At $3 \mathrm{dpf}$, the angles of DAC were measured using ImageJ. Mutant fish (curly up) and controls were assessed in the same manner. Additionally, PKD2 morphant and mutant fish were imaged under polarized light through $6 \mathrm{dpf}$ to observe the birefringence patterns of the muscle and overall body shape. The angles of DAC were measured and compared with those of the control fish. To assess the temporal activity of polycystin, its function was inhibited at various stages of somitogenesis with photomorpholino, a compound that can be activated by UV-light exposure at various time points. Degrees of DAC were subsequently measured and compared with control fish. This study was conducted under the regulations of the NYIT IACUC.

Data Analysis: The myotome lengths within the ventral, center, and dorsal segments of the fish as well as the angles of DAC were measured manually using ImageJ. The myotomes were designated relative to the cloaca. Counts of nuclei within individual myotome fibers were also performed manually. For each fish, three fibers were randomly selected within segments $(-1), 0$, and $(+1)$ for a nuclear count. Analysis of the birefringence patterns of the fish was qualitative. Statistical analysis was performed and data was plotted using Microsoft Excel. Statistical comparisons were performed using Student's t test, the parameters of which were two-tailed, assuming unequal variance. A calculated p-value of less than 0.05 was considered statistically significant. 
Results: The dorsal myotomes of PKD2 morphants had a significantly decreased rate of elongation compared with control fish as early as $1 \mathrm{dpf}$ $(\mathrm{p}<0.05)$; the PKD2 morphants also had shorter total trunk lengths $(\mathrm{p}<0.05)$ and taller myotomes along the dorsoventral axis $(\mathrm{p}<0.05)$. The quantity of nuclei per muscle fiber did not differ between the ventral and dorsal segments of the morphant fish, which was also seen in the controls; however, aberrant segmentation, characterized by boundary defects, was uniquely present in the PKD2 morphants' dorsal myotomes. In contrast, LY-treated fish had a significantly reduced number of nuclei in the dorsal segments compared with control fish $(p<0.001)$. Interestingly, PKD2 morphant fish that were treated with LY produced greater angles of DAC than those of both morphant fish alone and control fish $(\mathrm{p}<0.05$, $\mathrm{p}<0.05$ ); however, the PKD2 mutants (curly up) with and without LY-treatment did not produce curvature that differed from the control fish ( $p>0.05, p>0.05$ ). With respect to observation of the fish under polarized light, both morphant and mutant PKD2 zebrafish exhibited a segmental lack of muscle birefringence by $2 \mathrm{dpf}$ related to DAC, which was not seen in control fish. This pattern persisted through $6 \mathrm{dpf}$. Also, inhibition of polycystin early in somitogenesis (i.e. $80 \%$ epiboly) yielded greater DAC than later in somitogenesis (i.e. 8-10 somites and prim-5) $(\mathrm{p}<0.05)$. The angles of DAC at each embryonic stage differed from both the controls and each other.

Conclusion: Our results suggest that DAC in PKD2 morphants is driven by a decreased rate of elongation of the dorsal myotome. This is in contrast to the initial hypothesis of decreased nuclear proliferation of the dorsal myotome segments. Rather, the trunk lengths and heights of the morphant fish suggest that the underlying mechanism of DAC is related to a defect in $\mathrm{CE}$, since $\mathrm{CE}$ involves a coupled narrowing of tissue along a dorsoventral axis and lengthening along an anteroposterior axis. The segmental loss of birefringence in PKD2 morphants and mutants, along with the early effects of polycystin inhibition with the photomorpholino, suggests that polycystin plays an important role in muscle maturation during embryogenesis. We were, however, limited by assessing only three embryonic stages with the photomorpholino. The PI3K signaling pathway is likely involved with this phenotypic manifestation as well, since inhibition of both induced DAC. To our knowledge, this study is the first to suggest a direct effect of defective polycystin on aberrant skeletal muscle maturation, as previous literature has only indicated the presence of polycystin-1 expression in skeletal muscle tissue. Largely, we propose that polycystin plays a novel developmental role spanning multiple tissues, demonstrating an interconnectivity of body systems through one gene product. Future studies should aim to further evaluate the mechanism underlying $\mathrm{CE}$ defects as well as the relationship between PI3K and polycystin.

\section{References}

1. Mangos, S, Lam, PY, Zhao, A, Liu, Y, Mudumana, S, et al. The ADPKD genes $p k d 1 \mathrm{a} / \mathrm{b}$ and $\mathrm{pkd} 2$ regulate extracellular matrix formation. Disease Models \& Mechanisms. 2010;3(5-6): 354-365.

2. Patel, V, Chowdhury, R, Igarashi, P. Advances in the pathogenesis and treatment of Polycystic Kidney Disease. Current Opinion in Nephrology and Hypertension. 2009;18(2): 99

3. Peters, DJM, Van De Wal, A, Spruit, L, et al. Cellular localization and tissue distribution of polycystin-1. The Journal of Pathology. 1999;188(4):439-446. doi:10.1002/(sici)1096-9896(199908) 188:43.0.co;2-p.

4. Tada, M, Heisenberg, CP. Convergent extension: using collective cell migration and cell intercalation to shape embryos. Development. 2012;139(21):3897-3904. doi:10.1242/ dev. 073007 .

5. Vasilyev, A, Liu, Y, Hellman, N, Pathak, N, Drummond, IA. Mechanical Stretch and PI3K Signaling Link Cell Migration and Proliferation to Coordinate Epithelial Tubule Morphogenesis in the Zebrafish Pronephros. PLoS ONE. 2012;7(7). doi:10.1371/ journal.pone.0039992. 
$\star$ Poster No. *B48

Abstract No. 48

Category: Clinical

Research Focus Area: Impact of Osteopathic Manipulative Medicine (OMM) \& Osteopathic Manipulative Treatment (OMT)

\section{Correlation of Osteopathic} Examination of the Thoracic Spine With Ultrasound Examination

Jason Matthew Maddox, OMS IV ${ }^{1}$; Shirley Y. Chang, OMS IV²; Richard S. Dobrusin, DO ${ }^{2}$; Nathan

G. Nakken, $\mathrm{DO}^{2}$; Amy B. Stein, $\mathrm{MPH}^{3}$; Jeannine Noble, Master's Degree of Physical Therapy ${ }^{4}$; Randall L. Nydam, $\mathrm{PhD}^{4}$

${ }^{1}$ Midwestern University Arizona College of Osteopathic Medicine, Glendale, AZ; ' 2 Department of Osteopathic Manipulative Medicine, Midwestern University Arizona College of Osteopathic Medicine, Glendale, AZ; ${ }^{3}$ Department of Research \& Sponsored Programs, Midwestern University Arizona College of Osteopathic Medicine, Glendale, AZ; ${ }^{4}$ Department of Assessment, Midwestern University Arizona College of Osteopathic Medicine, Glendale, AZ

Statement of Significance: There has been literature published regarding using ultrasonography as a clinical tool to evaluate segmental motion for the cervical, lumbar, and sacral regions in the osteopathic clinical setting. However, no research has yet been published that compares osteopathic structural examination of the thoracic vertebral region to ultrasonographic examination. This study seeks to determine whether there is a significant correlation between osteopathic palpation and ultrasonographical measurements, as well as examine reliability between individual clinical examinations and ultrasound measurements.

Research Methods: Participants were volunteers recruited from the Midwestern University student body. A non-toxic, non-permanent marker was used to mark the skin. Two osteopathic physicians
(OMM1, OMM2) separately performed structural examinations by palpating T2-T5 transverse processes to determine vertebral rotation. Two trained sonographers (US1, US2) separately scanned and measured the distance from the tip of the spinous process to the adjacent transverse processes of the vertebral segment below. Demographic variables were summarized with mean and SD. Interexaminer reliability was assessed with percent agreement, Cohen's kappa, and Fleiss' kappa. Recruitment and protocols were approved by the MWU Institutional Review Board.

Data Analysis: Our results showed that when using two examiners for ultrasound, there is fair agreement for the overall "most significant" segmental rotation of the upper thoracic spine, with Cohen's Kapp at 0.27, with range of 0.09, 0.45, and total agreement percentage at a $52 \%$. For osteopathic examination, there is no statistic agreement for the overall upper thoracic spine, with a Cohen's Kappa at $0.05(0.0,0.27)$, and $32 \%$. Both US1 and US2 had slight agreement with one of the OMM examiners (OMM2), with Cohen's kappa of $0.14(0.0,0.33), 33 \%$ and 0.11 $(0.0,0.30), 30 \%$, respectively. However, if we analyzed each segment individually, we see that at T3, US1 and US2 has slight agreement at 0.20 $(0.02,0.37)$ and $48 \%$, while OMM1 and OMM2 did not show agreement $[0.01(0.0,0.12)]$, but had $44 \%$ agreement. There is slight agreement between US1 and OMM2 [0.10 $(0.03,0.23)]$. At T4, US1 and US2 again has slight agreement at $0.2(0.02,0.38)$ and $48 \%$, and OMM 1 vs OMM 2 did not have agreement with $0.08(0.0,0.24)$ and $39 \%$. There are no significant US and OMM agreements. At T5, US1 and US2 had moderate agreement at $0.44(0.27,0.60)$, or $64 \%$. OMM 1 and OMM2 had slight agreement at $0.12(0.0$, 0.28 ), or $42 \%$. There are no significant US and 
OMM agreements to each other. Fleiss' Kappa compares all raters at once: US1, US2, OMM1, and OMM2 (66 participants and 4 raters). This Fleiss' Kappa indicates at T3 is $-0.0512,0.0287$ at T4, and 0.0794 at T5, indicating low levels of agreements.

Results: Our results showed that ultrasound (US) had fair agreement for the overall most prominent segmental rotation of the upper thoracic spine. Osteopathic (OMM) palpation revealed no agreement for the overall most prominent vertebral rotation. Segment-specific vertebral analysis revealed slight agreement between US1 and OMM2 at the T3 vertebral segment, slight agreement between US1 and US2 at T4, and moderate agreement between US1 and US2 and slight agreement between OMM1 and OMM2 at T5. There are no significant ultrasound and OMM agreements to each other.

Conclusion: Osteopathic structural palpation had low interexaminer reliability, which is consistent with the literature. Ultrasound was found to have demonstrably better, albeit still relatively low, interexaminer reliability. We caution that palpation alone is not sufficiently reliable for diagnosis. We also find that ultrasound alone, while a demonstrably broadly applicable medical technology, is also likely not sufficiently reliable for diagnosis of thoracic somatic dysfunction. The low levels of correlation may stem from the fact that most participants were asymptomatic. Future study that looks at ultrasound and OMM examination correlation on a clinically symptomatic population may yield further findings. Furthermore, future studies should assess the pre- and post-treatment correlation of ultrasound and osteopathic examinations.

Acknowledgment/Funding Source: The research team thanks Dr. Dobrusin for his leadership and osteopathic knowledge, Dr. Nydam for his experience with research methodology, Dr. Nakken for his participation as a clinician, and Noble for her expertise with sonography. We also thank Dr. Stein for providing detailed statistical analysis and guidance. We thank Erich Berg for his help in methodology and data collection. We thank Karen Kin, Scott Messier, and Loren Swanson for their help in project implementation.

\section{References}

1. Seffinger, MA, American Osteopathic Association, eds Foundations of Osteopathic Medicine: Philosophy, Science, Clinical Applications, and Research. Fourth edition. Wolters Kluwer; 2018.

2. Shaw, KA, Dougherty, JJ, Treffer, KD, Glaros, AG. Establishing the Content Validity of Palpatory Examination for the Assessment of the Lumbar Spine Using Ultrasonography: A Pilot Study. J Am Osteopath Assoc. 2012;112(12):775. doi:10.7556/ jaoa.2012.112.12.775

3. Licciardone, JC, Nelson, KE, Glonek, T, Sleszynski, SL, des Anges Cruser null. Osteopathic manipulative treatment of somatic dysfunction among patients in the family practice clinic setting: a retrospective analysis. J Am Osteopath Assoc. 2005;105(12):537-544.

4. Brindise, J, Nelson, K, Kappler, R. Association Between Cervical and Thoracic Somatic Dysfunction Among Second-Year Osteopathic Medical Students. J Am Osteopath Assoc. 2014;114(7):540-548. doi:10.7556/jaoa.2014.107

Poster No. *B49

Abstract No. 49

Category: Basic Science

Research Focus Area: Chronic

Diseases \& Conditions

Quantitative Assessment of Human

Perivascular Adipocyte Tissue

\section{Phenotype}

Cathleen Huang, OMS III

University of New England College of Osteopathic

Medicine, Biddeford, ME

Statement of Significance: In healthy individuals, PVAT has a protective role by suppressing inflammation, while those with obesity, PVAT changes to cause an inflammatory effect. To understand the cellular basis of PVAT pathology, quantitative measurements to characterize human 
adipose tissues are necessary to help demonstrate the transformation of PVAT to resemble WAT. This research aligns with the mission of the American Heart Association and osteopathic physicians to build healthier lives. By bettering our understanding of PVAT and its impact on the vascular microenvironment to promote disease, physicians can significantly contribute to the cardiovascular clinical care of individuals with metabolic disease and improve their quality of life.

Research Methods: Samples used in this study were part of a larger project approved by the Institutional Review Board of Maine Medical Center (IRB \#1295440). For this project, I utilized samples from five donors undergoing coronary artery bypass graft (CABG) procedures and samples from five donors undergoing mitral valve repair (VR) procedures. From each donor, a sample of thoracic PVAT and subcutaneous WAT (SUBQ) was collected. I used both AdipoSoft (ImageJ) and AdipoCount (MATLAB) to quantify the samples. Once I established protocols for quantifying PVAT and WAT using each software, other users in the laboratory repeated the quantification of the same samples using the established protocols to establish precision of the methods.

Data Analysis: The frequency of adipocyte area, the average adipocyte size, and the number of adipocytes per 100,000 um2 were calculated. The percent stromal area was calculated by subtracting the total area of individual adipocytes from the total tissue area circumscribed. Comparison between independent raters' AdipoSoft data was determined by $\mathrm{t}$ test while comparison between independent raters' AdipoCount data was determined by 1-way ANOVA with Tukey's post-hoc test, both calculated in PRISM.

Results: The average adipocyte size of PVAT was less than those in SUBQ. The number of adipo- cytes per 100,000 um2 area was greater in PVAT compared with SUBQ in all CABG and VR samples. The stromal area was greater in PVAT samples compared with SUBQ. In the AdipoSoft protocol, a t test revealed no significant differences between independent raters' data. In the AdipoCount protocol, a 1-way ANOVA test with post-hoc revealed no significant differences between independent raters' data. Based on this analysis, we are confident in the precision of our methods.

Conclusion: Adipocyte size, number, and stromal area were quantified. We found that overall, adipocytes were smaller with more stromal adipose area in PVAT compared with SUBQ. This demonstrates that PVAT exhibits a different phenotype and role from WAT. Adipose analysis protocols showed high consistency when utilized with independent raters. The limitation of this project is the small number of independent raters; however, this is an initial project that can be replicated with more independent raters in the future. With a standardized protocol for quantifying human PVAT phenotype, scientists and physicians can utilize the established protocol to further investigate the transformative role of PVAT in obesity.

$\star$ Poster No. *B63

Abstract No. 63

Category: Clinical

Research Focus Area: Chronic

Diseases \& Conditions

The Usefulness of Radiograph in Assisting Diagnosis of COVID-19

Zachary Brennan, OMS II; Samantha B. Guerra, OMS II; Seelbinder A. Blake, OMS II; Lang L. Ashley, OMS II; Gerakinis J. Micah, OMS II

Michigan State University College of Osteopathic Medicine, East Lansing, MI 
Statement of Significance: We found that radiographs were an effective method of assisting in the diagnosis of COVID-19 and what findings were most common among positive radiographs. This will help osteopathic physicians determine the extent of infection and staging of the disease to determine whether Osteopathic Manipulative Treatment is indicated for these patients.

Research Methods: We assessed the first 100 patients in the Italian Society of Medical Radiology COVID-19 database. We only included the patients that had a radiograph, which left us with 61 patients. We then assessed whether the patients had positive findings on the radiograph or if they were considered negative by the radiologist. We then assessed what findings the radiologist noted on the radiograph.

We did not make our own assessment of the radiograph images but instead relied on the published radiology reports.

Data Analysis: We analyzed what percentage of the 61 patients used from the database (which all had radiographs and positive nasal swabs for COVID-19) were found to have positive radiographic findings noted by the radiologist. These findings were then further analyzed by the percentage of each unique positive finding noted. The data was assessed using a two proportion $\mathrm{z}$ test.

Results: $42(68.9 \%)$ of the 61 patients that were included, meaning that they had both a radiograph and a positive nasal swab for COVID-19, were deemed to have positive radiograph findings consistent with COVID-19. Of those patients, 30 $(71.4 \%)$ of the positive patients had parenchymal thickening on radiograph and $9(21.4 \%)$ of the positive patients had increased peribronchovascular interstitium. There were other findings on radiograph that were not statistically significant.
Conclusion: Radiographs can and have been used as a tool to assist with the diagnosis of COVID-19. When it is used, it is only going to show a positive finding in about $68.9 \%$ of cases, so while it may be used as a rule-in or to assess the degree of disease, it is not sufficient to be used as a rule-out test. When using radiographs, physicians are very likely to find parenchymal thickening on COVID-19 positive radiographs and somewhat likely to find increased peribronchovascular interstitium.

Acknowledgment/Funding Source: There are no acknowledgments or sources of funding for this research.

\section{Poster No. *B71}

Abstract No. 71

Category: Clinical

Research Focus Area: Osteopathic Philosophy

\section{Effects of the COVID-19 Pandemic on Osteopathic Medical Students' Screen Time \\ Cynthia O. Edimo, OMS III ${ }^{1}$; Jenzel K. Espares, OMS ${ } \mathrm{II}^{2}$; Nicole A. Falus, OMS $\mathrm{II}^{2}$; Sneha K. Bupathi, OMS ${ }^{\prime 2}$; Min-Kyung Jung, $\mathrm{PhD}^{3}$; Matthew B. Heller, $\mathrm{DO}^{2}$ ${ }^{1}$ New York Institute of Technology (NYIT), Old Westbury, NY; ${ }^{2}$ Department of Family, Medicine New York Institute of Technology College of Osteopathic Medicine, Old Westbury, NY; ${ }^{3}$ Department of Research, Old Westbury New York Institute of Technology College of Osteopathic Medicine, NY}

Statement of Significance: COVID-19 is a novel infectious disease that has led to the World Health Organization declaring it a pandemic in March 2020. Subsequently, osteopathic medical students were transitioned to an online form of learning. Studies have shown that an increase in screen time can predispose an individual to developing symptoms of Computer Vision Syndrome (CVS). 
CVS is a group of vision-related problems resulting from prolonged electronic screen use. Increased computer usage can lead to a decreased blink rate, placing individuals at higher risk of developing CVS, leading to likely reduced efficiency and productivity. Consequently, the transition to online resources for medical education may predispose medical students to developing CVS

Research Methods: This clinical trial was reviewed and approved by NYIT IRB (IRB number: BHS-1325). Participants were first and second year medical students matriculated at New York Institute of Technology College of Osteopathic Medicine. The study was conducted over one full academic year, with data collected on three separate occasions in August 2019, February 2020, and April 2020. For each of the three data collection times, students scheduled to meet in person with the research team to provide screen time data, gauge their visual acuity via a Snellen chart, and answer a questionnaire to screen for CVS symptoms. The amount of screen time was recorded using RescueTime, a program that provided quantitative data on how much screen time was spent on Apple and Windows devices. Participants downloaded RescueTime at the beginning of the study, and the screen time data was obtained during the 2 nd and 3rd visits via reports that were exported from the application. Once installed, RescueTime silently ran in the background of their devices and did not interfere with the participant's normal activities. Documentation of screen time was automated, reducing the risk for human error if participants were to estimate their screen time. The application also allowed for screen time data to be exported via print or email. All data from the questionnaire was entered into REDCap, a secure web applica- tion and HIPAA-compliant database designed for clinical and translational research.

Data Analysis: RescueTime was utilized to $\log$ participant data on their daily screen time usage. Average screen time was collected for the 9 months that the study was active (August 2019 to April 2020). Afterwards, statistical analysis was performed by a biostatistician to gather daily and monthly data on screen usage.

Results: A total of 33 participants were enrolled in this study with 22 participants included in this analysis as a result of loss to follow up. The mean screen time for the month of August was 2.4 hours per student per day. While the average screen time for participants increased to 5.9 hours in September and decreased to 4.9 hours in October, there was a consistent, considerable increase to greater than 6 hours in the last 4 months of the study. The average daily screen time in February was 6.0 hours. COVID-19 restrictions were enacted during the second week of March; at the end of the month, participants had an average screen time of 6.5 hours per day. April was the last month recorded, with an average screen time of 6.8 hours per day. Specifically, when noting the differences between March and April, there was approximately a $4.6 \%$ increase in screen time usage. Overall, the average screen time per day for medical students ranged from 2.4 to 6.8 hours from August 2019 to April 2020.

Conclusion: The results demonstrate an upward trend of usage in screen time among osteopathic medical students as the academic year progressed. Limitations included a small sample size and loss to follow-up with some participants. Additionally, the study protocol initially planned for a total of 3 visits; due to COVID-19 restrictions, all 
on-campus research-related activities were suspended prior to the participants' 3rd visit. As a result, the final visit was done virtually and visual acuity testing was not conducted. The increase in screen time is not conclusively caused by the increased demand for screen time related to COVID-19 restrictions as there may have been increased demands due to workload, examination deadlines and/or other factors. The differentiation between such variables was not examined in this study. Due to COVID-19 restrictions, many students had to undergo a massive adjustment in education, lifestyle and routine. Significantly increased screen time is a potential product of such change and may have affected this population of students in terms of visual acuity and other CVS-related symptoms. For this reason, there is an increased need for the medical student community to take part in preventative measures and specific management, especially if medical education in the face of a pandemic such as COVID-19 continues to be implemented in an online format.

Poster No. *H17

Abstract No. 17

Category: Clinical

Research Focus Area:

Musculoskeletal Injuries and

Prevention

\section{Arthrocentesis in Pediatric Septic} Arthritis of the Knee: A Case Study Kaitlyn Wright, OMS IV; Daniel Schlepphorst, OMS IV; Hanna S. Sahhar

Edward Via College of Osteopathic Medicine (South Carolina), Spartanburg, SC

Statement of Significance: Identifying and treating SA in a timely fashion is important due to potential adverse effects of infection, such as joint destruction or deformity. It is important to recognize the three etiologies of SA and recognize that a more indolent course may arise with certain pathogens. Osteopathic manipulative techniques used to promote lymphatic drainage of the knee, such as opening of the thoracic inlet and diaphragm and release of the pelvic diaphragm, would be beneficial early in the plan of care due to its musculoskeletal nature.

Research Methods: The patient was recruited to participate in this case study due to the rarity of the Aeromonas hydrophila SA and its resistance to the usual empiric antibiotics. We gathered data for the case report from electronic health records of the patient with informed consent from patient and parent and online searches in peer-reviewed published literature in PubMed.

Data Analysis: This case report assesses the importance of arthrocentesis in the management of pediatric SA due to its ability to accurately diagnosis the condition as well as the pathogen(s) and their respective sensitivities to many antibiotics. We analyzed consults and reports from health care professionals providing care, blood laboratory values, blood cultures and pathology reports, reports from radiologists for plain radiographs of the knee in three views and magnetic resonance imaging (MRI) of the knee without contrast, and photographs of the area through the extent of his hospital stay.

Results: Plain radiographs on initial presentation showed soft tissue swelling but no joint effusion, foreign bodies, or osseous erosion. MRI of the right knee showed a developing abscess in the anteromedial aspect of the knee along with a small effusion. Blood cultures acquired from multiple lines was did not show any growth after five days. Laboratory results from blood displayed an increase in inflammatory marker, C-reactive protein (CRP) throughout the patient's week-long hospital stay 
despite a decrease in white blood cell count after initiation of empiric antibiotics. Synovial fluids aspirated days later from the affected joint showed cloudy, yellow fluid without crystals. Gram stain of the synovial fluid showed Gram-negative rods, and bacterial culture was evident for Klebsiella pneumoniae and Aeromonas hydrophila. Of which, Aeromonas was resistant to Cefepime.

Conclusion: We strongly believe that the patient would have benefited in care (quality and length of stay) if an arthrocentesis would have been performed earlier regardless of negative imaging in his care to establish diagnosis with a specific pathogen and its sensitivity to antibiotics. While Aeromonas hydrophila is normally sensitive to a fourth-generation cephalosporin or fluoroquinolone, which was prescribed prior to receiving results of susceptibilities, this patient had an infection with Aeromonas bacteria with resistance to Cefepime. This knowledge did change the plan of care and led to the patient being discharged from the hospital. Knowing the culture and sensitivities days before would have led to antibiotic prescription changes, better control of infection, and shorter stay in the hospital. Though it is unlikely the patient had septic arthritis on his initial presentation, there should be a high index of suspicion for contiguous spread as the infection seemed to spread.

\section{References}

1. Danaher, PJ, Mueller, WP. Aeromonas hydrophila septic arthritis. Mil Med. 2011;176(12):1444-1446. doi:10.7205/ milmed-d-11-00211

2. Gigante, A, Coppa, V, Marinelli, M, Giampaolini, N, Falcioni, D, Specchia, N. Acute osteomyelitis and septic arthritis in children: a systematic review of systematic reviews. Eur Rev Med Pharmacol Sci. 2019;23(2 Suppl):145-158. doi:10.26355/ eurrev_201904_17484

3. Pääkkönen, M. Septic arthritis in children: diagnosis and treatment. Pediatric Health Med Ther. 2017;8:65-68. Published 2017 May 18. doi:10.2147/PHMT.S115429

4. Doganis, D, Baka, M, Tsolia, M, et al. Multifocal Aeromonas Osteomyelitis in a Child with Leukemia. Case Rep Infect Dis. 2016;2016:8159048. doi:10.1155/2016/8159048
Poster No. H24

Abstract No. 24

Category: Basic Science

Research Focus Area: Chronic

Diseases \& Conditions

\section{Implications and Significance of} Mercury in Rice

Tracee Guthrie, OMS I ${ }^{1}$; Erin Collins, OMS I'; Gurleen Kaur, OMS I'; Brooke Benjamin, OMS I ${ }^{2}$

${ }^{1}$ Edward Via College of Osteopathic Medicine

(Virginia), Blacksburg, VA; ${ }^{2}$ University of

Pikeville-Kentucky College of Osteopathic Medicine,

Pikeville, KY

Statement of Significance: In this study, 29 samples of rice were compared for mercury content (12 from Thailand, 6 from India, 6 from China, compared with 5 control samples from the USA). Samples ranged from 0.18 to $6.01 \mathrm{ng}$ of element / g. Further research is needed to establish standards for mercury toxicity. These standards are necessary, especially for breastfeeding mothers who are exposing their children to the mercury they might be consuming on a daily basis. An exposure that has not been explored in infant development over the course of time. The incorporation of these standards into labeling and recognition of the signs of toxicity can increase the chances of proper treatment times to avoid severe damages.

Research Methods: In this study, 29 samples of rice were compared for mercury content (12 from Thailand, 6 from India, 6 from China, compared with 5 control samples from the USA). The content of 29 rice samples was analyzed for methyl mercury using mass spectrometry (ICP-MS). Samples ranged from 0.18 to $6.01 \mathrm{ng}$ of element / g. Further research is needed to establish standards for mercury toxicity.

Data Analysis: The sample from China (Sample ID: 143-27) was the highest with $6.01 \mathrm{ng}$ of element / $g$ sample. This rice was not white or brown, it was 'other' coloured. There were many samples from Thailand, seven of the top ten, 
which had the highest mercury amount. There were two samples from the United States in the top ten, with $3.71 \mathrm{ng}$ of element/ $\mathrm{g}$ sample (Sample ID:143-4) and $2.84 \mathrm{ng}$ of element/ g sample (Sample ID: 143-25). These samples were part of the control. There were no samples from India in the top ten. With regards to colours, eight out of the top ten samples were white rice, one brown and one 'other'. The lowest concentration of methyl mercury was $0.18 \mathrm{ng}$ of element / $\mathrm{g}$ sample (Sample ID: 143-29). This sample was from China and 'other' coloured.

Results: The data (Table 1) shows that the rice grown in the United States (control) did not significantly differ in concentration of mercury when compared with rice grown in other countries in Asia. The hypothesis stems from the data collected. The top ten rice samples included two from the United States, which were supposed to be a control. No rice grown in India was in the top ten. Of the top ten countries, seven samples were from Thailand, two from the United States, and the highest sample from China. This could have been due to the specific region the rice was cultivated in China. This area could have had a higher fossil fuel emission due to industrial plants in the same area [33]. Of the top ten samples with the highest mercury content, eight were white, one was brown and one was an 'other' colour. Colour, relating to the outer coating of the rice could be a source of further investigation. Does rice colour correlate with higher or lower amounts of mercury across certain regions? If so, what could be the cause of the higher or lower readings?

The amount of $\mathrm{MeHg}$ in rice samples shown in (Table 1) demonstrates the need for further research on mechanisms of mitigation of $\mathrm{MeHg}$ in rice growing and preparation. There is a lack of qualitative data on tolerable daily mercury levels recommendations supplied by the FDA, thus it is difficult to inform the at risk populations expecting mothers, neonates, and infants) on provisional tolerable weekly intake, PWTI [36].

Conclusion: The paper highlights the importance of improved integration of warning labels for high risk populations due to increasing evidence of high amounts of mercury in rice and rice products. It was originally thought that fish and rice from areas of high pollution would have increased mercury when compared with areas that have decreased pollution, but this study proves that rice in all areas, regardless of amount of pollution or type of pollution, have high levels of mercury. Thus, as rice becomes more of a staple in food items consumed, it is imperative that action needs to be taken to reduce exposure and harm and that more research needs to be conducted to conclude the sources of high mercury levels in specific regions.

Solutions for addressing exposure to this ubiquitous metal and its subsequent diverse health implications include further research, public education, and increased regulations for mercury levels in rice. Research assessing which sources of rice have high mercury concentrations so causes can be more closely examined are needed. Public education to mothers, nullipara women, and all humans about the significance of mercury exposure in foods and or breastmilk will potentially mitigate the amount of exposure. Limiting levels of mercury in rice to ameliorate transfer and exposure to this heavy metal should be analysed.

Acknowledgment/Funding Source: This research was funded by the Edward Via College of Osteopathic Medicine, Blacksburg, Virginia, 24060.

\section{References}

1. Jingying $\mathrm{Xu}$, Andrea Garcia Bravo, Anders Lagerkvist, Stefan Bertilsson, Rolf Sjöblom et al (2015) Sources and remediation techniques for mercury contaminated soil. Environ Int 74: 42-53. [Crossref]

2. Joint, F. A. O. (2010) Seventy-second meeting, Rome, 16-25 February 2010: summary and conclusions. Rome: Joint FAOMHO Expert Committee on Food Additives, 16 p. JECFA/72/SC, 2010. 
Poster No. * $\mathrm{H} 25$

Abstract No. 25

\section{Category: Health Services}

Research Focus Area: Osteopathic Philosophy

\section{Rural Hospital Closures Extend Transit Times Focusing in Obstetrics Gabriel Ward, MA, BS ${ }^{1}$; Susan Meacham, PhD, RD ${ }^{2}$ ${ }^{1}$ Edward Via College of Osteopathic Medicine (South Carolina), Spartanburg, SC; ${ }^{2}$ Edward Via College of Osteopathic Medicine (Virginia) Biomedical Affairs and Research, Blacksburg, VA}

Statement of Significance: Rural hospital closures have been increasing throughout the country over the last ten years. If the rate of closures continues at the current rate 11.7 million patients may be impacted. Studies have found that hospital closures, more prevalent in southern states, nearly double the mean EMS travel times in rural zip codes in comparison to urban zip codes. The service area of Virginia incorporates a total population of $8,517,586$ with over $12 \%$ living in rural areas. In southwest Virginia, there have been three rural hospital closings since 2013. A study is needed to characterize and document EMS in Southwest Virginia to inform institutions and hospitals with the most accurate and correct information regarding recent hospital closure impacts.

Research Methods: The research methodology entailed the collection of detailed information from previous research, statistical analysis of hospital closures based of public records, public data banks, and analysis of previous studies done on the topic. All referenced articles were found using keyword PubMed searches looking for articles analyzing rural hospital closures. The keyword searches began with the term rural hospital closures initially then later transitioned to hospital closures effect on emergency transport services. Information was also gathered by a strategic search within governmental run websites and databanks, so the most accurate information could be obtained on population sizes and hospital distributions. Once, all information was gathered a comparison was done between the statistics seen in counties without hospital closures to the counties that experienced hospital closures, specifically looking at how the mean EMS activation time was affected.

Data Analysis: In southwest Virginia the rural hospital closures (Mountain View Regional Hospital, Pioneer Community Hospital, and Lee Regional Medical Center) force longer travel times for patients and EMS personnel. Those that went to Mountain View regional are currently able to go to another local hospital within a 10-mile radius. Patients previously seen at Pioneer Community and Lee Regional Hospitals now have to travel anywhere from 15-30 miles, which, in rural Virginia can be a travel time of 25 or more minutes. These travel times are incredibly important when it comes to emergency services, transport times by first responders, and outcomes in critical situations. Our review of literature was unable to find peer-reviewed data to inform medical professionals on the status of EMS in our rural area. Our review of literature was unable to find peer-reviewed data to inform faculty and students on the status of the rural areas served by our medical professionals in southwest Virginia. The geographic boundaries of our search was expanded for important transferable data was applicable to our study because of the lack of peer-reviewed research on the topic in Southwest Virginia. 
Results: A study conducted between 2010-2016 in Utah $(n=58487)$ provided data on the impact of rural hospital closures. Utah's Bureau of Emergency Services reported that for every oneminute increase in response time by EMS there was an increase in mortality by $8 \%$ and $17 \%$. Researchers found if a hospital system closed, that system's EMS services close as well; six percent of all EMS are hospital based. The impact of hospital closures was evident in the Utah study; an increase in mean EMS total activation time in minutes from $75.3 \pm 0.26$ (SD) to 79.0 \pm 0.27 (SD) in counties prior and post rural hospital closure. Nationally, a decrease from $55 \%$ to $44 \%$ in hospital obstetrics services was found in rural counties between 2004-2014. A large cohort study (1086 counties) found that counties losing hospital-based OB/GYN care not adjacent to an urban area experienced a $3 \%$ increase in births occurring in hospitals without $\mathrm{OB} / \mathrm{GYN}, 0.67 \%$ being preterm births. This national cohort study using the National EMS Information System (NEMSIS) not only showed an increase in calls but an increase in incidences; revealing counties with rural hospital closures had an increase in all aspects of the EMS care timeline. Comparing prior to hospital closures to post hospital closures shows that after closures occurred, the transport times in counties without hospital closures had widespread decrease in all EMS timeline statistics while counties that experiences hospital closures increased.

Conclusion: Emergent calls involving obstetrics are important because they often can have two lives on the line. With EMS services being specific to the location and protocols depending on services available; it is important to have the data organized and accurate for each designated area throughout the nation. The study done in Utah provides a good prototype for the analysis and understanding of rural hospital closures on the efficiency of emer- gency medical services. It is baseline for future studies and illustrates the comprehensive research protocol to follow in other areas of the country; that will help policy makers understand the specific impacts, fiscal health, and investments needed to preserve access to critical emergency services. This will improve the performance of EMS calls and highlight where the country needs to improve health care infrastructure for best care of every citizen. As osteopathic students with interest in the regions where we plan to serve, e.g., Southwest Virginia, we conclude that a 'call to action' is needed to inform policy and practice. We recommend a study be conducted to gather data on EMS need in rural communities where rural hospital closures have occurred. The data will inform institutions with the most accurate and correct information regarding recent hospital closures. This would specifically improve obstetrics care, one of the most critical specialties of care, and improve care for all citizens requiring other medical services in rural regions.

Poster No. *H39

Abstract No. 39

Category: Health Services

Research Focus Area: Osteopathic Philosophy

Underserved Students Learning and Perceptions Towards Careers in Science, Technology, Engineering, Mathematics, and Medicine (STEMM) Alexis Nicole Antonopoulos, OMS IV; Angela Pluguez, OMS IV; Jamey Moore, OMS IV; Rebekah Cook, OMS IV; Troy Camarata, PhD New York Institute of Technology College of Medicine at Arkansas State University, Jonesboro, AR

Statement of Significance: Osteopathic medical students from New York Institute of Technology College of Osteopathic Medicine (NYITCOM) in 
Jonesboro, Arkansas who are members of the Student Osteopathic Medical Association (SOMA) volunteered to participate in an after-school outreach program under the framework of NOD, a national program sponsored by SOMA. This program is designed to instill ambition and confidence in underrepresented children who may feel that careers in STEMM are unattainable. This research was inspired, in part, by a retrospective study of highquality afterschool programs, which found that participating children see an improvement in their selfperception, an increase in positive social behavior, and a decrease in problem behaviors (1).

Research Methods: The National Outreach for Diversity (NOD) after-school program curriculum consisted of six interactive bi-weekly sessions highlighting different Science, Technology, Engineering, Math, and Medicine (STEMM) topics. At the beginning and at the conclusion of the NOD after-school program, Microsociety Magnet School students were administered a ten question anonymous survey to ascertain their perceptions toward careers and topics in STEMM in order to determine if participants portrayed more optimistic viewpoints at the conclusion of the program. Participants were also asked to answer a series of two multiple choice questions at the beginning and at the conclusion of each session which provided a measure of participant understanding of the topics discussed in each session. Lastly, participants were given a reflective, constructive journal question at the conclusion of each session which provided insight into each of the participants individual thoughts. New York Institute of Technology College of Osteopathic Medicine (NYITCOM) Institutional Review Board (IRB) approval was obtained (IRB protocol \#BHS1443) under exemption status. A letter of agreement was obtained from the study site, Microsociety Magnet School in Jonesboro, AR.
Data Analysis: A Mann-Whitney U test was utilized to determine if there was a difference in average student performance on pre-session and post-session journal questions. Student's journal questions were converted to numerical values representing whether the student's score decreased, remained the same, or improved. A Mann-Whitney U test was again utilized to determine if there was a difference in students' perceptions toward STEMM before the outreach program began and after the outreach program concluded. Student's survey question answers were recorded numerically as $1,2,3,4$, or 5 points, representing the responses "disagree a lot", "disagree a little", "I don't know", "agree a lot", and "agree a little". All data was run through the Statistical Package for the Social Sciences (SPSS). A p-value below 0.05 was considered a statistically significant value.

Results: Throughout the six National Outreach for Diversity (NOD) after-school program sessions, an average of 14 students participated in each session. Students who did not complete the pre-session questions, post-session questions, or both were not included in the study. Analysis of pre-session and post-session journal questions showed an increase in the mean overall student performance, $(\mathrm{U}=2480.5, \mathrm{Z}=-2.22, \mathrm{P}=0.026)$. Analysis of survey questions revealed that mean overall student perception toward the survey topic "People do not need to understand science because it is not a part of daily life" significantly improved $(\mathrm{U}=64, \mathrm{Z}=-2.07, \quad \mathrm{P}=0.039)$. However, the other nine survey questions were statistically insignificant with p-values $>0.05$, therefore, observed data distribution did not significantly vary from the expected data distribution for these questions. As the after-school program progressed, several new students were added to the program and several students were switched to different programs or ceased participation in 
after-school programming. Therefore, measuring students' perceptions toward STEMM topics overall was limited secondary to varying student populations.

Conclusion: The National Outreach for Diversity (NOD) after-school program had an immediate impact on underserved and underrepresented elementary-aged students' understanding of topics and occupations in Science, Technology, Engineering, Math, and Medicine (STEMM). The program created a partnership and mentorship between elementary school students and medical students within the Jonesboro community. As NOD is a national program sponsored by SOMA, we hope to inspire additional SOMA chapters and like-minded medical students to create sustainable community-based outreach programs for underserved and underrepresented students across the country. The NOD program at NYITCOM in Jonesboro, Arkansas has continued to increase their classroom size, along with the number of medical students involved in conducting each session. We hope to also explore online education and mentoring options to accommodate school closures secondary to the pandemic.

Acknowledgment/Funding Source: We thank the New York Institute of Technology College of Osteopathic Medicine (NYITCOM) students, faculty, and staff who participated in and helped facilitate the National Outreach for Diversity (NOD) after-school program, especially Rajendram Rajnarayanan, M. Sc., PhD, Tracy Owens, Ed. D., and Sloane Kelley, MS. We also thank Microsociety Magnet School faculty Ariel Murray and Mandy Zipfel, Assistant Principal, for coordinating the after-school program.

\section{Reference}

1. Krishnamurthi, A., Ballard, M., \& Noam, G. G. (2014). Examining the impact of afterschool STEM programs. Retrieved 2020, from https://files.eric.ed.gov/fulltext/ED546628.pdf. $\star$ Poster No. ${ }^{*} \mathrm{H} 47$

Abstract No. 47

Category: Clinical

Research Focus Area: Impact of

Osteopathic Manipulative Medicine (OMM) \& Osteopathic Manipulative Treatment (OMT)

AOA Grant Number: 19157991539

\section{The Effect of Facial Effleurage on} Serum Complement C3 During Acute Rhinosinusitis: A Randomized,

\section{Controlled Study}

Kyle Burke, OMS IV ${ }^{1}$; Chelsea Weidman, $\mathrm{MS}^{2}$; Jillian H. Bradley, $\mathrm{PhD}^{2}$

${ }^{1}$ Edward Via College of Osteopathic Medicine (South Carolina), Spartanburg, SC; ${ }^{2}$ Department of Microbiology and Immunology, Edward Via College of Osteopathic Medicine (South Carolina), Spartanburg, $\mathrm{SC}$

Statement of Significance: Osteopathic Manipulative Treatments (OMTs) have long been used, but their mechanisms of action have been underexplored. We aimed to elucidate the immunological mechanism of Facial Effleurage (FE), a type of OMT, in acute rhinosinusitis. FE uses rhythmic pressure to refresh facial lymphatics and improve sinus mucus clearance; this could have a direct impact on the symptoms and immune system in rhinosinusitis patients. Acute rhinosinusitis, a common condition in ambulatory settings, is often treated with antibiotics. OMT offers adjunct or alternative non-pharmacological interventions. Understanding if FE treatment in rhinosinusitis patients can ameliorate symptoms by impacting their immune system is crucial to FE's more widespread use.

Research Methods: The Edward Via College of Osteopathic Medicine Institutional Review Board approved all protocols. Ninety-one participants between the ages of 18 and 69 years were 
recruited from a community health clinic in Spartanburg, South Carolina. They were either determined to be healthy or diagnosed with acute rhinosinusitis by physicians at the clinic. Healthy participants were randomly assigned no treatment, Facial Effleurage, or sham treatment. Rhinosinusitis participants were randomly assigned antibiotics (standard-of-care), Facial Effleurage, sham treatment, antibiotics and Facial Effleurage, or antibiotics and sham treatment. The sham treatment mimics the patient's and physician's movements and hand placements of the Facial Effleurage procedure. However, the sham treatment does not use therapeutic pressure and, therefore, provides a control for Facial Effleurage without offering any therapeutic benefit. Antibiotics were started after treatment on the same day of their first visit. Serum samples were obtained prior to treatment, 1 hour following treatment, and at a 1-week follow-up visit. ELISAs were performed to obtain serum $\mathrm{C} 3$ concentrations.

Data Analysis: Within Microsoft Excel, Student's $t$ tests were performed to assess for significance between serum $\mathrm{C} 3$ concentrations in each group. Fisher's Exact Test was used to determine if there was a significant difference between the demographic categories of each treatment group. Graphs were created using GraphPad Prism 8.4.1 software. Significance was defined as a p-value $<$ 0.05 .

Results: Rhinosinusitis patients who received Facial Effleurage had significantly lower serum C3 concentrations 1 hour after treatment compared with those who received the sham treatment $(-373$ $\pm 778 \mu \mathrm{g} / \mathrm{mL}$ vs $761 \pm 1346 \mu \mathrm{g} / \mathrm{mL}, \mathrm{p}=0.0320$ ). There were no other significant differences in average serum $\mathrm{C} 3$ concentrations in any other treatment group 1-hour post-treatment or 7-10 days post-treatment. There was no significant dif- ference in average baseline C3 serum concentrations $(\mathrm{p}=0.418)$ between healthy $(2510$ $\pm 1353 \mu \mathrm{g} / \mathrm{mL})$ and $\mathrm{RS}(2258 \pm 1476 \mu \mathrm{g} / \mathrm{mL})$ participants.

Conclusion: Facial Effleurage changes serum C3 concentrations as soon as 1-hour following treatment. This novel finding is the first evidence of a potential mechanism of action for Facial Effleurage and how it can provide this benefit to patients suffering from acute rhinosinusitis. We suggest Facial Effleurage, and other forms of Osteopathic Manipulative Treatment, should be used as an additional acute rhinosinusitis treatment. Further studies are needed to determine how long the immunological impact persists as well as other mechanisms of action of Facial Effleurage.

A limitation of this study was the low sample size per group; more samples could have provided more statistical power to better detect additional significant differences between treatment groups. Another limitation is that we measured peripheral serum C3 concentrations and not what is occurring directly in the nasal mucosa, which might paint an even more interesting picture of the benefit of Facial Effleurage.

Acknowledgment/Funding Source: We would like to thank our collaborators at Palmetto Proactive Healthcare, Drs. Chris McCarthy and Jerome Aya-Ay, for allowing us to recruit patients at their clinics and Drs. Matthew Cannon and Oshea Escamilla for performing the OMT needed for this study. We would like to thank our additional collaborators: Alexis Stoner, MPH, Ph.D.; Randal Gregg, Ph.D.; Ning Cheng, Ph.D. The work was generously funded by The American Osteopathic Association (Grant Numbers 2031815722 and 19157991539). 
Poster No. *H53

Abstract No. 53

Category: Health Services

Research Focus Area: Osteopathic Philosophy

\section{Physician Experiences With}

Telemedicine During the COVID-19

Pandemic: A Practitioner Survey

Using a Novel Telehealth Provider

Inventory

Nicole Alexa Falus, OMS II; Alyssa A. Curcio, OMS II;

Emily R. Dries, OMS III; Todd J. Cohen, MD

New York Institute of Technology (NYIT), Old

Westbury, NY

Statement of Significance: For most providers, and especially osteopathic physicians, the basis of diagnosis is through the physical examination, which has been limited due to the use of telemedicine during the pandemic. The Centers for Medicare and Medicaid Services issued the 1135 Medicaid Waiver on $3 / 6 / 20$, expanding the criteria for telemedicine reimbursement, aiding the rapid shift of clinics to a telemedicine model. The findings from this study can be used to develop more efficient and beneficial ways of using telemedicine, while decreasing stressors and burden for both patients and providers. Ultimately, improving the telemedicine experience can improve physician satisfaction, reduce physician burn-out, and improve patient health outcomes by avoiding viral exposure.

Research Methods: This survey received IRB approval (BHS-1567) from New York Institute of Technology (NYIT).

This study is an anonymous, voluntary survey that asks providers about their experiences with telemedicine. Questions include demographics, prior experience with telemedicine, thoughts about the current system, and opinions on how to improve for the future. Qualitative and quantitative data was collected and analyzed. The researchers were unable to find a validated survey that would accurately capture these unique circumstances; therefore, this research team developed a novel telemedicine inventory in order to assess provider experiences

Physicians at NYIT College of Osteopathic Medicine (NYITCOM) Academic Health Care and Family Health Care Center, and NYITCOM alumni physicians were emailed a REDCap survey to complete. Inclusion criteria included being an attending physician and having seen a patient using telemedicine since the COVID-19 pandemic started (March 2020).

Data Analysis: Responses were analyzed and descriptive statistics were calculated using JASP statistical software when appropriate. Qualitative "write-in" responses were studied for overarching themes and grouped into appropriate categories for analysis. A total of 84 physician responses were analyzed, including physicians from 21 different specialties and 16 different states.

Results: Only $22.6 \%$ of physicians had used telemedicine prior to the pandemic, and 58.4\% would not have considered offering telemedicine had it not been for COVID-19. 85.7\% of respondents were still offering in-person visits, and $64.4 \%$ reported offering more in-person encounters than telemedicine.

$57.1 \%$ of respondents were either satisfied or very satisfied with their telemedicine platform, and the vast majority $(72.6 \%)$ found their platform easy or very easy to use.

Reported benefits of telemedicine included the enhancement of patient safety by minimizing patient exposure to the SARS-CoV-2 virus (89.3\%), convenience $(77.4 \%)$, patient satisfaction $(52.4 \%)$, appointments running more timely $(36.9 \%)$, and monetary reimbursement $(10.7 \%)$. 
The reported difficulties were poor signal or internet connection $(60.7 \%)$, less thorough encounters $(50 \%)$, less personal encounters $(47.6 \%)$, concerns about errors in care $(20.2 \%)$, difficulty using the system $(20.2 \%)$, patient dissatisfaction $(6 \%)$, and limited time during encounter $(4.8 \%)$. Other common points of dissatisfaction included lack of physical examination of patients and patient difficulty with telemedicine.

Overall, $59.5 \%$ of respondents reported being satisfied or very satisfied with their telemedicine encounters. $81 \%$ of participants indicated they would continue to use telemedicine during the pandemic. When asked about continued use after the pandemic ends, $51.2 \%$ responded they will continue its use, $14.3 \%$ will not, and $34.5 \%$ are unsure.

Conclusion: Based on this study, the vast majority of participants reported using telemedicine for the first time during the COVID-19 pandemic. Although telemedicine use has increased, the data shows that most study participants were still offering in-person patient encounters, and still having more in-person visits than telemedicine encounters.

Over half of the physicians reported satisfaction with their telemedicine encounters, but there are still plenty of improvements to be made. Some current areas for improvement include lifting legal restrictions, particularly the requirements for both physician and patient to be in the same state. Other common sources of dissatisfaction included limited ability to perform physical examinations, and technological problems including patient difficulty with telemedicine platforms, and signal/ connection issues. Improvement in these areas should be addressed to improve physician and patient experiences.

Limitations include non-response bias and smaller sample size, restricting statistical analysis of geographical region, specialty and platform use. Specific platform satisfaction could not be assessed due to many participants using multiple platforms. Future studies can include a larger sample size from more regions and specialties.

Future research areas can include methods of increasing the thoroughness of examination of patients, such as at home technology, and improving patient education on telemedicine use.

Acknowledgment/Funding Source: We would like to acknowledge and thank the NYITCOM physicians and alumni that participated in this survey. No funding sources were used for this research.

\section{References}

1. Almathami, H, Win, K, Vlahu-Gjorgievska, E. Barriers and Facilitators That Influence Telemedicine-Based, Real-Time, Online Consultation at Patients' Homes: Systematic Literature Review. J Med Internet Res. 2020;22(2):e16407. doi:10.2196/ 16407

2. Donaghy, E, Atherton, H, Hammersley, V et al. Acceptability, benefits, and challenges of video consulting: a qualitative study in primary care. British Journal of General Practice. 2019;69 (686):e586-e594. doi:10.3399/bjgp19×704141

3. Grover, J, Smith, B, Williams, J et al. Novel Use of Telemedicine by Hurricane Evacuation Shelters. Prehospital Emergency Care. 2020:1-9. doi:10.1080/10903127.2020.1723756

4. Thiyagarajan, A, Grant, C, Griffiths, F, Atherton, H. Exploring patients' and clinicians' experiences of video consultations in primary care: a systematic scoping review.

5. Waller, M, Taylor, L, Portnoy, J. Benefits and risks of incorporating virtual visits into an allergy/immunology practice. Allergy Asthma Proc. 2020;41(2):76-81. doi:10.2500/ aap.2020.41.190022 
Poster No. *H62

Abstract No. 62

Category: Basic Science

Research Focus Area: Chronic

Diseases \& Conditions

\section{An Investigation of Corpora Amylacea} in the Spinal Cord of Aged Individuals

Andrew Thomas Matthew Colebank, OMS II'; Waeya

T. Lin, OMS $\|^{2}$; Joyce Morris-Wiman, $\mathrm{PhD}^{2}$

${ }^{1}$ West Virginia School of Osteopathic Medicine,

Lewisburg, WV; ${ }^{2}$ Department of Biomedical Sciences,

West Virginia School of Osteopathic Medicine,

Lewisburg, WV

Statement of Significance: Corpora amylacea

(CA) were first described by Purkinje in 1837 as spherical inclusions in aged human cerebrum.

Since then, CA were discovered within the context in neurodegenerative diseases. Morphologically, CA from cerebrum (CCA) are spherical, translucent bodies, ranging from 10-50 um in diameter. Histochemical analysis indicates CCA are basophilic polysaccharides, identified by periodic acid-Schiff reagent (PAS). Proteins are a minor component of CA,normally associated with neuroinflammation. Within osteopathy's tenet of structure's relation to function, recent studies suggest CA form from astrocytic scavenging of cellular debris and act as "waste containers." Increased CCA may indicate that the process of elimination becomes faulty.

Research Methods: Participants: Spinal cord specimens were harvested from six female donors to the WVSOM Human Gift Program, ages 81-95. The tissue was cryoprotected, snap-frozen in isopentane cooled in liquid nitrogen, and stored at $-80^{\circ}$ cryosectioned at $14 \mu \mathrm{m}$. Sections were placed on contiguous slides or simultaneous histological and immunofluorescent-staining evaluation. Sections were stained with a modified Klüver-Barrera protocol for an assessment of white matter integrity and with PAS-staining to identify corpora amylacea. Consecutive sections were immunostained for the astrocyte marker GFAP, the neuronal marker MAP2,for markers of neurodegeneration, tau protein, amyloid, and ubiquitin.

Immunofluorescent-Staining: Cryosections were immunostained using standard protocols. Briefly, sections were rinsed in PBS and incubated first in $2 \mathrm{~N} \mathrm{HCl}$ for antigen retrieval, rinsed and then in $0.1 \%$ sodium borohydride for reduction of background autofluorescence. Section were incubated in primary antibody (GFAP, MAP2, amyloid, tau, ubiquitin) overnight at $40 \mathrm{C}$ and then with appropriate secondary antibody at room temperature for $2 \mathrm{hrs}$. Images were acquired using a Zeiss MRm digital camera and Axiovision software and were examined for the presence or absence of immunostained CA.

Data Analysis: PAS-staining: Cryosections were stained using an alcoholic PAS method to establish the identity of the particles as CA. Digital images of sections were acquired using a Leica Aperio system and analyzed using ImageJ and Imagescope software. In Image J images were converted to an standard 8-bit grayscale version and utilizing ROI Line Function, the entire diameter and area of the cross sectioned images and regions of interest were recorded including: the dorsal horn, ventral horn, lateral corticospinal tract, fasciculus cuneatus, and fasciculus gracilus. Particle analysis included measurements of number of CA per area, individual Feret diameter in $\mu \mathrm{m}$, and individual area in $\mu \mathrm{m} 2$.

Klüver-Barrera staining: Cryosections were rinsed in PBS, and then stained in Luxol fast blue overnight. Stain was differentiated in lithium carbonate and sections counterstained in hematoxylin. Digital images of sections were acquired using a Leica Aperio system and analyzed using ImageJ software. In ImageJ, utilizing ROI Function tools, 
the entire diameter and area of the cross sectioned image and regions of interest were recorded; central and peripheral regions were delineated. Using the Color Segmentation tool the image was thresholded, CA were highlighted and counted.

Statistical comparisons between groups and were made using a two way ANOVA and, when significant $(\mathrm{p}<0.05)$, were examined pair-wise using post-hoc comparisons (Statistica, Tibco).

Results: Immunofluorescent-labeling of spinal cord section revealed that CA were immunospositive for a neuronal marker (MAP2). In contrast, the CA were surrounded by GFAP but not immunospositive for GFAP. These observations would indicate that CA may contain neuronal but not astrocytic components. Immunostaining for the markers of neurodegeneration revealed spinal CA included tau and ubiquitin, not amyloid. Amyloid showed patchy, amorphous distribution throughout all regions, although the number of 'patches' increased in older participants. Although CA did not include amyloid, several instances of newly forming CA within regions of amyloid were observed. Not all CA were positive for the previous, and CA within the peripheral posterior funiculi were more likely to be immunospositive for these markers, indicating heterogeneity of spinal cord CA composition.

The number of CA detected differed significantly between the dorsal and ventral horns, as well as the peripheral and central portions. As expected, the numbers of CA were increased within the fasciculus gracile and cuneate compared with the lateral corticospinal tract. No significant difference was detected between CA numbers in the fasciculus gracile to cuneate. The average diameter of CA in the spinal cord was $16.2+0.16 \mu \mathrm{m}$, similar to reported diameters of CCA. The average diameter of CA did not differ significantly between regions. However, there were significant differences in CA diameter and regional distribution between participants.

Conclusion: The results of this study indicate that the PAS-positive inclusion bodies within the spinal cord of aged individuals are of a similar size, distribution, and composition to corpora amylacea described in the cerebral hemispheres of aged individuals. Their location within the spinal cord periphery and the inclusion of tau and ubiquitin within the inclusions, suggest that, as in the cerebrum, $\mathrm{CA}$ in the spinal cord function in eliminating debris.

\section{Acknowledgment/Funding Source:}

This study was funded from an intramural grant from the West Virginia School of Osteopathic Medicine for Summer Student Research Program. The team would like to acknowledge Dr. Jennifer Bannister for her contribution to research in Spinal Cord Neurodegenerative Changes With Post-Polio Syndrome and Dementia, as well as Dr. Peter Ward for his excellent, encouraging dissection in handling the human body donors.

\section{References}

1. Auge, E., et al, Corpora amylacea in human hippocampal brain tissue are intracellular bodies that exhibit a homogeneous distribution of neo-epitopes. Sci Rep, 2019. 9(1): p. 2063

2. Auge, E., et al, New perspectives on corpora amylacea in the human brain. Sci Rep, 2017. 7: p.41807.

3. Cana, A., et al, Characterization of Periodic Acid-Schiff-Positive Granular Deposits in the Hippocampus of SJL/J Mice. Toxicol Pathol, 2015. 43(5): p. 737-42

4. Jacob, L., Boisserand, L.S.B., Geraldo, L.H.M. et al. Anatomy and function of the vertebral column lymphatic network in mice. Nat Commun 10, 4594 (2019). https://doi.org/10.1038/ s41467-019-12568-w

5. Cavanagh, J.B., Spinal corpora amylacea and motor neuron disease: a quantitative study. J Neurol Neurosurg Psychiatry, 1998. 65(4): p. 488-91.

6. Bannister, J. and J. Morris-Wiman, Spinal Cord Neurodegenerative Changes With Post-Polio Syndrome and Dementia JAOA, 2018, 118: p. e101-e202. 
Poster No. *H70

Abstract No. 70

Category: Clinical

Research Focus Area: Osteopathic Philosophy

\section{Prevalence of Computer Vision}

Syndrome (CVS) Symptoms on

\section{Osteopathic Medical Students}

Jenzel Espares, OMS III'; Cynthia O. Edimo, OMS

$\mathrm{III}^{2}$; Sneha K. Bupathi, OMS $\mathrm{I}^{2}$; Nicole A. Falus, OMS

$\mathrm{II}^{2}$; Min-Kyung Jung, $\mathrm{PhD}^{3}$; Matthew B. Heller, $\mathrm{DO}^{2}$

${ }^{1} \mathrm{New}$ York Institute of Technology (NYIT), Old

Westbury, NY; ${ }^{2}$ Department of Family Medicine,

New York Institute of Technology (NYIT), Old

Westbury, NY; ${ }^{3}$ Department of Research, New York

Institute of Technology (NYIT), Old Westbury, NY

Statement of Significance: The American

Optometric Association describes Computer

Vision Syndrome as a group of eye and visionrelated problems that result from prolonged computer, tablet, e-reader and cell phone use. Increased computer usage can lead to a decreased blink rate, and those who develop CVS will likely have reduced efficiency and productivity of work. The NYIT College of Osteopathic Medicine as an institution specifically takes pride in its integration of technology into the medical education curriculum and provides school-issued iPads to students when they first start medical school. Consequently, this emphasis on technology inadvertently places its students at increased risk of manifesting CVS or symptoms related to it.

Research Methods: This clinical trial was reviewed and approved by NYIT IRB (IRB number: BHS-1325). Participants were first and second year medical students matriculated at New York Institute of Technology College of Osteopathic Medicine. The study was conducted over one full academic year, with data collected on three separate occasions in August 2019, February 2020, and April 2020. For each of the three data collection times, students scheduled to meet in person with the research team to provide screen time data, gauge their visual acuity via a Snellen chart, and answer a questionnaire to screen for CVS symptoms. The amount of screen time was recorded using RescueTime, a program that provided quantitative data on how much screen time was spent on Apple and Windows devices. Participants downloaded RescueTime at the beginning of the study, and the screen time data was obtained during the 2 nd and 3 rd visits via reports that were exported from the application. Once installed, RescueTime silently ran in the background of their devices and did not interfere with the participant's normal activities. Documentation of screen time was automated, reducing the risk for human error if participants were to estimate their screen time. The application also allowed for screen time data to be exported via print or email. All data from the questionnaire was entered into REDCap, a secure web application and HIPAA-compliant database designed for clinical and translational research.

Data Analysis: CVS symptom incidence and frequency obtained from a reliable and validated questionnaire were exported from REDCap as a Microsoft Excel document, which were then plotted as a graph to illustrate results and to draw comparisons between the first and second visits.

Results: A total of 27 participants completed all of the administered questionnaires. Regarding the CVS symptoms, 16 symptoms were monitored throughout the study, and study participants indicated the presence or absence of the symptom as well as the frequency and severity of their symptom if it was indeed present. The symptoms that were asked in the questionnaire are the following: burning in eyes, itching in eyes, feeling of a foreign body, tearing of eyes, excessive blinking, eye redness, eye pain, heavy eyelids, 
eye dryness, blurred vision, double vision, difficulty focusing for near vision, increased sensitivity to light, colored halos around objects, feeling that sight is worsening, and headache. The questionnaire also asked participants if they had undergone any change in their ocular prescription between visits (ex. Getting a new pair of glasses). The data that we obtained showed an increase in prevalence of the following symptoms on the second visit compared with the first visit: Burning in eyes $(59.3 \%$ vs $44.4 \%)$, Itching in eyes $(63 \%$ vs $59.3 \%)$, Excessive blinking (33.3\% vs $25.9 \%)$, Heavy eyelids (63\% vs $44.4 \%)$, Eye dryness $(76.8 \%$ vs $59.2 \%)$, Increased sensitivity to light (37\% vs $25.9 \%$ ), and Headache (70.4\% vs $51.8 \%$ ). The percentage values noted above represent the number of participants who confirmed that they experienced that specific symptom. At the second visit, $18.5 \%$ of participants indicated that they had undergone a change in their ocular prescription since their last visit.

Conclusion: Based on the results, the most common symptoms reported by the study participants were headache and dry eyes, which were present in more than $70 \%$ of the study pool during the second visit.

The study is limited by a small sample size and loss to follow-up. A total of 33 participants were enrolled at the beginning of the study, and that number dropped to 27 by the end of the academic year. Participants were considered to have been lost to follow-up when they did not attend an in-person visit and thus did not take the questionnaire administered by the research team. Also, the study protocol had originally planned for three questionnaires (one per visit); due to COVID-19 restrictions that were enacted in March 2020, all on-campus research-related activities were suspended prior to the participants' 3rd visit.
Therefore, the final visit (scheduled for April 2020) was conducted in a virtual manner. While screen time data was obtained from most study participants, we were unable to administer the questionnaire for participants to fill out. Due to the high prevalence of CVS symptoms in osteopathic medical students as demonstrated by this study, it would be appropriate to educate incoming students on preventative measures and draft an osteopathic treatment protocol to mitigate the incidence of these symptoms. Further investigation on the effects of screen time on visual acuity is warranted as COVID-19 restrictions have placed an increased demand for screen time in medical education.

Poster No. *H72

Abstract No. 72

Category: Clinical

Research Focus Area: Chronic Diseases \& Conditions

A Comprehensive Virtual Plan for People With Parkinson's Disease During the COVID-19 Pandemic Nicholas Piniella, OMS II ${ }^{1}$; Laura Ketigian, OMS III'; Kaylie McGivney, OMS II'; Austin Dukat, OMS II'; Samatha Lui, SPT ${ }^{2}$; David Bolocboloc, SPT'; Rosemary Gallagher, PT, DPT, PhD²; Adena Leder, DO, FAAN $^{3}$

${ }^{1}$ New York Institute of Technology (NYIT), Old

Westbury, NY; ${ }^{2}$ Department of Physical Therapy, New York Institute of Technology (NYIT), Old Westbury, NY; ${ }^{3}$ Department of OMM, New York Institute of Technology (NYIT), Old Westbury, NY

Statement of Significance: COVID-19 required adaptation of our existing programs to maintain the physical and mental health of PWP. Anxiety and depression, combined with elevated stress levels and lack of usual support systems during the crisis had the potential to create feelings of 
isolation. Therefore, we transitioned to a virtual platform; virtual RSB (vRSB) classes and support groups (SG) and added Rock Steady Buddies: one-on-one conversations with students, in our holistic approach to retain physical and mental health, and quality of life (QoL). These programs pertain to the osteopathic tenets of health maintenance and preventive services, and the interrelationship of structure and function in our offerings of equitable, safe and cost-efficient care.

Research Methods: Our in-person RSB (pRSB) classes and weekly SG transitioned to a synchronous virtual platform using Zoom after shelter-in-place orders were initiated in March 2020. Rock Steady Boxing is non-contact boxing that challenges both physical and cognitive domains with the aim of improving both motor and non-motor symptoms of PD. Two one-hour vRSB classes, of differing intensities, began three times per week. The weekly SG cover topics ranging from shared personal experiences, to COVID-19 informational sessions, to guest speakers. The Rock Steady Buddies program was developed with the aim to promote feelings of social connectedness, particularly for our patients that live alone. It started in April 2020 and partners NYITCOM students with PWP to engage in remote one-on-one conversations, with a suggestion of one hour per week. All programs are currently ongoing. In June 2020 , a custom online survey was distributed to participants from all three programs and was available for two weeks. Included were males and females with a diagnosis of PD, or with an atypical form of PD, who participated in at least one of the programs. Questions explored the barriers, facilitators, satisfaction and motivation when participating in each of our virtual programs. The survey also included a measure of depression, the Patient Health Questionnaire-9
(PHQ-9), and the Parkinson's Disease Questionnaire-39 (PDQ-39) as a measure of QoL. This study was exempt by the Institutional Review Board at NYITCOM.

Data Analysis: Information was collected using Research Electronic Data Capture (REDCap), a secure database. The survey was completely anonymous with no identifying information collected. Data was exported into excel to calculate percentages and means for descriptive statistics and are reported here. Although the survey was sent to anyone who has ever expressed interest in the RSB program, this was not our target audience for this study. Therefore, data was analyzed only from responders who had attended at least one of our virtual programs $(n=100)$.

Results: A 47\% response rate was attained. Respondents had a mean age of 68.7 years (SD 8.6 , range $47-86 y)$, most were male (75\%) and caucasian (93\%). On average, respondents reported 7.6 new/worsening PD symptoms since March, with 94\% reporting at least one. The most common were anxiety (57\%), sleep problems (49\%) and gait impairment (47\%). Many reported faster progression of their PD (43\%), worsening $\operatorname{mood}(47 \%)$ and feelings of loneliness/isolation (62\%) despite $85 \%$ having a good support system. Thirty nine attended vRSB; of 38 who participated in both pRSB and vRSB, 61\% attended vRSB more often than pRSB, possibly due to a $52 \%$ decrease in barriers to attending. Overall, $82 \%$ were satisfied with vRSB; and 59\% would continue vRSB post-pandemic. Respondents agreed that vRSB improved their motor symptoms ( $80 \%)$, mood (72\%), and helped the same or more than pRSB (41\%). Of the 16 who report virtual SG attendance, $100 \%$ rated the groups as moderately or extremely helpful. Common reasons respondents did not attend virtual SG include already having support (26\%) 
and it being a different experience when remote (26\%). Twenty three respondents participated in Buddies; $86 \%$ report that it improved mood and $68 \%$ reported feeling less isolated/lonely. Most reported satisfaction with Buddies (96\%) and would continue the program after the pandemic (82\%). The most common reason for not participating was feeling awkward (25\%). PHQ-9 revealed $29 \%$ of scores $\geq 9$. Of 31 completed PDQ-39's, scores averaged 20.3.

Conclusion: Age and comorbid conditions, such as in PWP, are leading risk factors of mortality from COVID-19. With strict social isolation rules enacted, PWP report worsening symptoms such as anxiety and difficulty with walking, which underscores the importance of maintaining social relationships and staying physically active during the current pandemic. The comprehensive in-person programs at NYITCOM quickly transitioned to online platforms for this purpose. Despite reports of some barriers to participation in the programs, as a whole they were successful. Exercise compliance increased due to accessibility of vRSB, with similar satisfaction and perceived efficacy as pRSB. Virtual SG spread timely, accurate health information, which is paramount amid concerns of misinformation. With rising levels of stress and anxiety felt by PWP, the Buddies program proved to be effective and efficient, highlighting the impact of interpersonal relationships on overall mind, body and spirit. Our limitations include a small sample size - however, the study is currently ongoing - and response bias. Responders tended to be an active, intelligent and motivated group. The current study supports NYITCOM's success in piloting a multifaceted virtual program for PWP, which has proven both feasible and satisfying, and resulted in perceived benefits across the physical, social, and psychological aspects of PD.

\section{Reference}

1. Kurtis, M. M., \& Mir, P. (2020, June 3). International Parkinsons and Movement Disorder Society. Treating COVID-19 in PD and other Movement Disorders: A Review of Drug Interactions [Slides]. https://www.movementdisorders.org/MDS/Education/ Workshops-Conferences/MDS-Webinars/Live/Treating-COVID19-in-PD-and-other-Movement-Disorders-A-Review-of-DrugInteractions.htm

(๑) 2020 American Osteopathic Association 